\title{
Measurements of Higgs boson production cross sections and couplings in the diphoton decay channel at $\sqrt{\mathrm{s}}=13 \mathrm{TeV}$
}

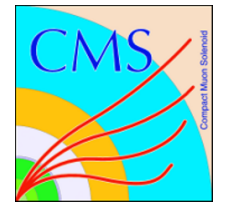

\section{The CMS collaboration}

E-mail: cms-publication-committee-chair@cern.ch

ABSTRACT: Measurements of Higgs boson production cross sections and couplings in events where the Higgs boson decays into a pair of photons are reported. Events are selected from a sample of proton-proton collisions at $\sqrt{s}=13 \mathrm{TeV}$ collected by the CMS detector at the LHC from 2016 to 2018, corresponding to an integrated luminosity of $137 \mathrm{fb}^{-1}$. Analysis categories enriched in Higgs boson events produced via gluon fusion, vector boson fusion, vector boson associated production, and production associated with top quarks are constructed. The total Higgs boson signal strength, relative to the standard model (SM) prediction, is measured to be $1.12 \pm 0.09$. Other properties of the Higgs boson are measured, including SM signal strength modifiers, production cross sections, and its couplings to other particles. These include the most precise measurements of gluon fusion and vector boson fusion Higgs boson production in several different kinematic regions, the first measurement of Higgs boson production in association with a top quark pair in five regions of the Higgs boson transverse momentum, and an upper limit on the rate of Higgs boson production in association with a single top quark. All results are found to be in agreement with the SM expectations.

KEYwORDs: Hadron-Hadron scattering (experiments), Higgs physics

ArXiv EPrint: 2103.06956 


\section{Contents}

1 Introduction 2

2 The CMS detector 3

3 Analysis strategy 4

3.1 The STXS framework 4

3.2 Analysis categorisation 6

4 Data samples and simulated events $\quad 8$

5 Event reconstruction $\quad 10$

$\begin{array}{lll}5.1 & \text { Photon reconstruction } & 10\end{array}$

$\begin{array}{lll}5.2 & \text { Photon identification } & 12\end{array}$

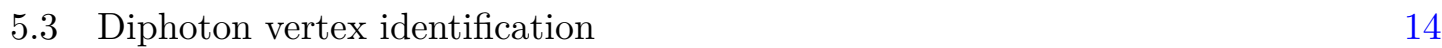

$\begin{array}{ll}5.4 \text { Additional objects } & 15\end{array}$

6 Event categorisation $\quad 16$

$\begin{array}{lll}6.1 & \text { Event categories for ggH production } & 17\end{array}$

6.2 Event categories for VBF production 22

6.3 Event categories for hadronic VH production 24

6.4 Event categories for leptonic VH production 26

6.5 Event categories for top quark associated production 30

6.6 Summary of the event categorisation 33

$\begin{array}{lll}7 & \text { Statistical procedure } & 36\end{array}$

$\begin{array}{lll}7.1 & \text { Signal model } & 38\end{array}$

$\begin{array}{ll}7.2 & \text { Background model } \\ \end{array}$

8 Systematic uncertainties $\quad 39$

$\begin{array}{lll}8.1 \text { Theoretical uncertainties } & 39\end{array}$

8.2 Experimental uncertainties 43

9 Results $\quad 45$

9.1 Signal strength modifiers $\quad 45$

9.2 Simplified template cross sections 48

9.3 Coupling modifiers 53

10 Summary $\quad 56$

$\begin{array}{ll}\text { The CMS collaboration } & 67\end{array}$ 


\section{Introduction}

Since the discovery of a Higgs boson (H) by the ATLAS and CMS collaborations in 2012 [1$3]$, an extensive programme of measurements focused on characterising its properties and testing its compatibility with the standard model (SM) of particle physics has been performed. Analysis of data collected during the second run of the CERN LHC at $\sqrt{s}=13 \mathrm{TeV}$ has already resulted in the observation of Higgs boson production mechanisms and decay modes predicted by the SM [4-7]. The most precise measurements are obtained by combining results from different Higgs boson decay channels. Such combinations have enabled the total Higgs boson production cross section to be measured with an uncertainty of less than $7 \%[8,9]$. All reported results have so far been consistent with the corresponding SM predictions.

In the SM, the $\mathrm{H} \rightarrow \gamma \gamma$ decay has a small branching fraction of approximately $0.23 \%$ for a Higgs boson mass $\left(m_{\mathrm{H}}\right)$ around $125 \mathrm{GeV}$ [10]. However, its clean final-state topology with two well-reconstructed photons provides a narrow invariant mass $\left(m_{\gamma \gamma}\right)$ peak that can be used to effectively distinguish it from background processes. As a result, $\mathrm{H} \rightarrow \gamma \gamma$ is one of the most important channels for precision measurements of Higgs boson properties. Furthermore, it is one of the few decay channels that is sensitive to all principal Higgs boson production modes.

The results reported in this paper build upon previous analyses performed by the CMS collaboration [11, 12]. Here, the data collected by the CMS experiment between 2016 and 2018 are analysed together. The resulting statistical power of the combined data set improves the precision on existing measurements and allows new measurements to be made. The structure of this analysis is designed to enable measurements within the simplified template cross section (STXS) framework [10]. Using this structure, various measurements of Higgs boson properties can be performed. These include SM signal strength modifiers, production cross sections, and the Higgs boson's couplings to other particles. Measurements of all these quantities are reported in this paper.

The STXS framework provides a coherent approach with which to perform precision Higgs boson measurements. Its goal is to minimise the theory dependence of Higgs boson measurements, lessening the direct impact of SM predictions on the results, and to provide access to kinematic regions likely to be affected by physics beyond the SM (BSM). At the same time, this approach permits the use of advanced analysis techniques to optimise sensitivity. Reducing theory-dependence is desirable because it makes the measurements both easier to reinterpret and means they are less affected by potential updates to theoretical predictions, making them useful over a longer period of time [13]. The results presented within the STXS framework nonetheless depend on the SM simulation used to model the experimental acceptance of the signal processes, which could be modified in BSM scenarios.

The strategy employed in this analysis is to construct analysis categories enriched in events from as many different kinematic regions as possible, thereby providing sensitivity to the individual regions defined in the STXS framework. This permits measurements to be performed across all the major Higgs boson production modes, including gluon fusion $(\mathrm{ggH})$, vector boson fusion (VBF), vector boson associated production (VH), production 
associated with a top quark-antiquark pair $(\mathrm{t} \overline{\mathrm{t}} \mathrm{H})$, and production in association with a single top quark ( $\mathrm{tH})$.

In addition to measurements within the STXS framework, this paper contains several other interpretations of the data. The event categorisation designed to target the individual STXS regions also provides sensitivity to signal strength modifiers, both for inclusive Higgs boson production and for individual production modes, as well as measurements within the $\kappa$-framework [14].

The paper is structured as follows. The CMS detector is described in section 2. An overview of the STXS framework is given in section 3, together with a summary of the overall strategy of this analysis. In section 4, details of the data and simulation used to design and perform the analysis are given. The reconstruction of candidate $\mathrm{H} \rightarrow \gamma \gamma$ events is described in section 5 , before the event categorisation procedure is explained in section 6 . The techniques used to model the signal and background are outlined in section 7 , with the associated systematic uncertainties listed in section 8 . The results are presented in section 9, with tabulated versions provided in HEPDATA [15]. Finally, the paper is summarised in section 10 .

\section{The CMS detector}

The central feature of the CMS apparatus is a superconducting solenoid of $6 \mathrm{~m}$ internal diameter, providing a magnetic field of $3.8 \mathrm{~T}$. Within the solenoid volume are a silicon pixel and strip tracker, a lead tungstate crystal electromagnetic calorimeter (ECAL), and a brass and scintillator hadron calorimeter (HCAL), each composed of a barrel and two endcap sections. The ECAL consists of 75848 lead tungstate crystals, which provide coverage in pseudorapidity $|\eta|<1.48$ in the barrel region and $1.48<|\eta|<3.00$ in the two endcap regions. Preshower detectors consisting of two planes of silicon sensors interleaved with a total of 3 radiation lengths of lead are located in front of each EE detector. Forward calorimeters extend the $\eta$ coverage provided by the barrel and endcap detectors. Muons are detected in gas-ionisation chambers embedded in the steel flux-return yoke outside the solenoid.

Events of interest are selected using a two-tiered trigger system [16]. The first level, composed of custom hardware processors, uses information from the calorimeters and muon detectors to select events at a rate of around $100 \mathrm{kHz}$ within a fixed time interval of less than $4 \mu \mathrm{s}$. The second level, known as the high-level trigger, consists of a farm of processors running a version of the full event reconstruction software optimised for fast processing, and reduces the event rate to around $1 \mathrm{kHz}$ before data storage [17].

The particle-flow (PF) algorithm [18] aims to reconstruct and identify each individual particle (PF candidate) in an event, with an optimised combination of information from the various elements of the CMS detector. The energy of photons is obtained from the ECAL measurement. The energy of electrons is determined from a combination of the electron momentum at the primary interaction vertex as determined by the tracker, the energy of the corresponding ECAL cluster, and the energy sum of all bremsstrahlung photons spatially compatible with originating from the electron track. The energy of muons is 
obtained from the curvature of the corresponding track. The energy of charged hadrons is determined from a combination of their momentum measured in the tracker and the matching ECAL and HCAL energy deposits, corrected for zero-suppression effects and for the response function of the calorimeters to hadronic showers. Finally, the energy of neutral hadrons is obtained from the corresponding corrected ECAL and HCAL energies.

For each event, hadronic jets are clustered from these reconstructed particles using the infrared and collinear safe anti- $k_{\mathrm{T}}$ algorithm $[19,20]$ with a distance parameter of 0.4. Jet momentum is determined as the vectorial sum of all particle momenta in the jet, and is found from simulation to be, on average, within 5 to $10 \%$ of the true momentum over the whole transverse momentum $\left(p_{\mathrm{T}}\right)$ spectrum and detector acceptance. Additional proton-proton interactions within the same or nearby bunch crossings (pileup) can contribute additional tracks and calorimetric energy depositions to the jet momentum. To mitigate this effect, charged particles identified to be originating from pileup vertices are discarded and an offset correction is applied to correct for remaining contributions. Jet energy corrections are derived from simulation to bring the measured response of jets to that of particle level jets on average. In situ measurements of the momentum balance in dijet, photon + jet, $\mathrm{Z}+$ jet, and multijet events are used to account for any residual differences in the jet energy scale between data and simulation [21]. The jet energy resolution amounts typically to $15-20 \%$ at $30 \mathrm{GeV}, 10 \%$ at $100 \mathrm{GeV}$, and $5 \%$ at $1 \mathrm{TeV}$ [21]. Additional selection criteria are applied to each jet to remove jets potentially dominated by anomalous contributions from various subdetector components or reconstruction failures.

The missing transverse momentum vector $\vec{p}_{\mathrm{T}}^{\text {miss }}$ is computed as the negative vector $p_{\mathrm{T}}$ sum of all the PF candidates in an event, and its magnitude is denoted as $p_{\mathrm{T}}^{\text {miss }}[22]$. The $\vec{p}_{\mathrm{T}}^{\mathrm{miss}}$ is modified to account for corrections to the energy scale of the reconstructed jets in the event.

A more detailed description of the CMS detector, together with a definition of the coordinate system used and the relevant kinematic variables, can be found in ref. [23].

\section{Analysis strategy}

\subsection{The STXS framework}

In the STXS framework, kinematic regions based upon Higgs boson production modes are defined. These regions, or bins, exist in varying degrees of granularity, following sequential "stages". At the so-called STXS stage 0, the bins correspond closely to the different Higgs boson production mechanisms. Events where the absolute value of the Higgs boson rapidity, $\left|y_{\mathrm{H}}\right|$, is greater than 2.5 are not included in the definition of the bins because they are typically outside of the experimental acceptance. Measurements of stage- 0 cross sections in the $\mathrm{H} \rightarrow \gamma \gamma$ decay channel were presented by the CMS collaboration in ref. [12]. Additionally, an analysis probing the coupling between the top quark and Higgs boson in the diphoton decay channel was recently performed by the CMS collaboration [24]. Several other stage-0 measurements in different decay channels have also been made by both the ATLAS and CMS collaborations [25-31]. Each experiment has also presented results combining the various analyses $[8,9]$. 


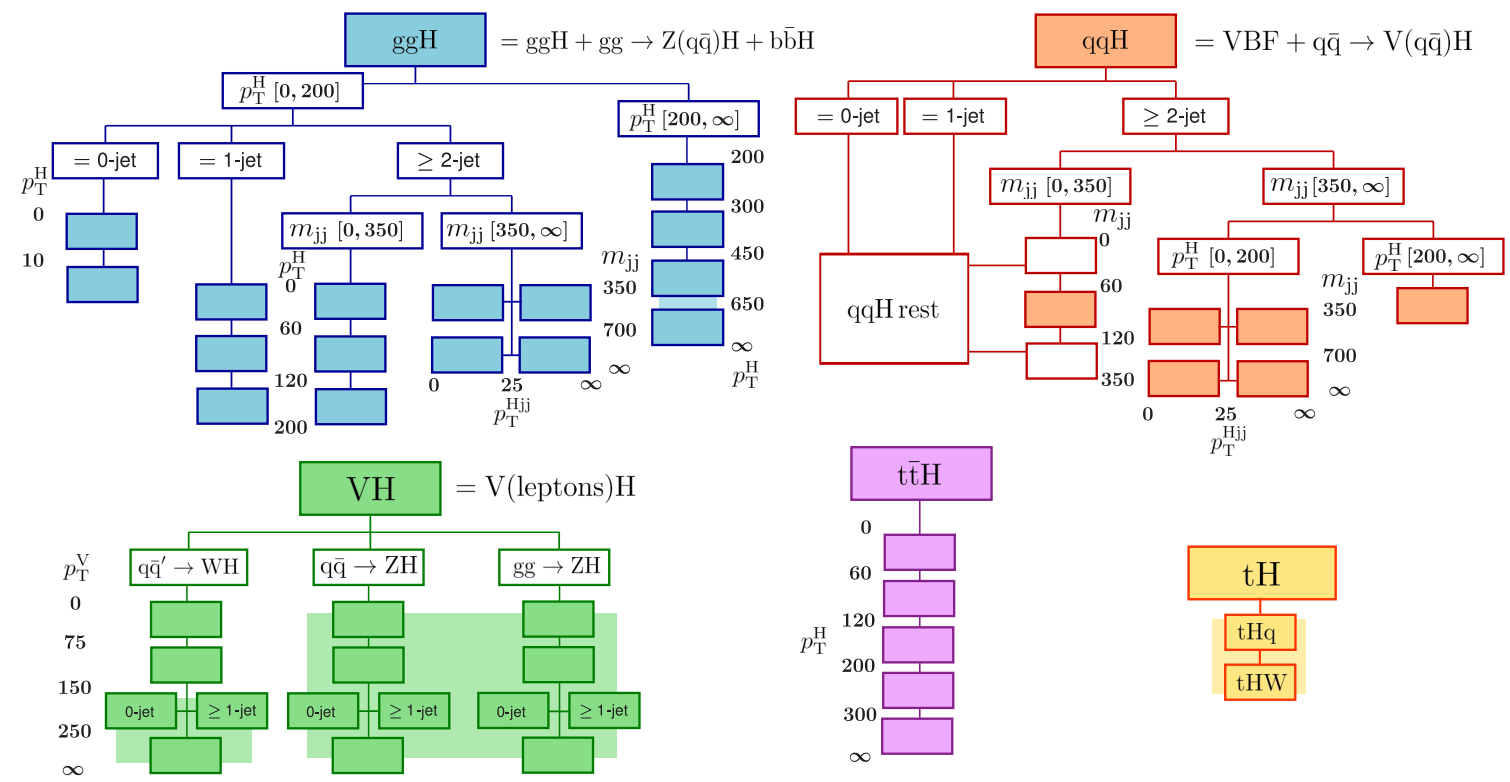

Figure 1. Diagram showing the full set of STXS stage-1.2 bins, adapted from ref. [10], defined for events with $\left|y_{\mathrm{H}}\right|<2.5$. The solid boxes represent each STXS stage-1.2 bin. The units of $p_{\mathrm{T}}^{\mathrm{H}}, m_{\mathrm{jj}}$, $p_{\mathrm{T}}^{\mathrm{Hjj}}$, and $p_{\mathrm{T}}^{\mathrm{V}}$ are in $\mathrm{GeV}$. The shaded regions indicate the STXS bins that are divided at stage 1.2, but are not measured independently in this analysis.

At STXS stage 1, a further splitting of the bins using the events' kinematic properties is performed [32]. This provides additional information for different theoretical interpretations of the measurements, and enhances the sensitivity to possible signatures of BSM physics. Furthermore, increasing the number of independent bins reduces the theorydependence of the measurements; within each bin SM kinematic properties are assumed, and thus splitting the bins allows these assumptions to be partially lifted.

Measurements at stage 1 of the framework have already been reported by the ATLAS collaboration [25, 26, 33]. Following these results, adjustments to the framework and its definitions were made, such that the most recent definition of STXS bins is referred to as STXS stage 1.2. The first measurement of STXS stage-1.2 cross sections was recently performed by the CMS collaboration [34].

The full set of STXS stage-1.2 bins is described below and an illustration is given in figure 1. The ggH region (blue) is split into STXS bins using the Higgs boson transverse momentum $\left(p_{\mathrm{T}}^{\mathrm{H}}\right)$, the number of jets, and additionally has a VBF-like region with high dijet mass $\left(m_{\mathrm{jj}}\right)$. This VBF-like region is split into four STXS bins according to $m_{\mathrm{jj}}$ and the transverse momentum of the Higgs boson plus dijet system $\left(p_{\mathrm{T}}^{\mathrm{Hjj}}\right)$. Events originating from $b \bar{b} \mathrm{H}$ production are grouped with the $\mathrm{ggH}$ production mode, as are those from gluon-initiated production in association with a vector boson $(\mathrm{ggZH})$ where the vector boson decays hadronically. The VBF and hadronic VH modes are considered together as electroweak qqH production (orange). Here the STXS bins are defined using the number of jets, $p_{\mathrm{T}}^{\mathrm{H}}, m_{\mathrm{jj}}$ and $p_{\mathrm{T}}^{\mathrm{Hjj}}$. The four STXS bins which define the $\mathrm{qqH}$ rest region are not explicitly probed in this analysis. The leptonic VH STXS bins (green) are split into three 
separate regions representing the $\mathrm{WH}, \mathrm{ZH}$, and gg ZH production modes, which are further divided according to the number of jets and the transverse momentum of the vector boson

$\left(p_{\mathrm{T}}^{\mathrm{V}}\right)$ that decays leptonically. The $\mathrm{t} \overline{\mathrm{t}} \mathrm{H}$ production mode (pink) is split only by $p_{\mathrm{T}}^{\mathrm{H}}$. Finally, the tH STXS bin includes contributions from both the tHq and tHW production modes. All references to STXS bins hereafter imply the STXS stage-1.2 bins. Further details on the exact definitions are contained in section 6, describing the event categorisation. All the production mechanisms shown in figure 1 are measured independently in this analysis.

\subsection{Analysis categorisation}

To perform measurements of Higgs boson properties, analysis categories must first be constructed where the narrow signal peak is distinguishable from the falling background $m_{\gamma \gamma}$ spectrum. The categorisation procedure uses properties of the reconstructed diphoton system and any additional final-state particles to improve the sensitivity of the analysis. As part of the categorisation, dedicated selection criteria and classifiers are used to select events consistent with the $\mathrm{tH}, \mathrm{t} \overline{\mathrm{t}} \mathrm{H}, \mathrm{VH}, \mathrm{VBF}$, and ggH production modes. This both increases the analysis sensitivity and enables measurements of individual production mode cross sections to be performed.

In order to measure cross sections of STXS bins individually, events deemed to be compatible with a given production mode are further divided into analysis categories that differentiate between the various STXS bins. For most production modes, the divisions are made using the detector-level equivalents of the particle-level quantities used to define the STXS bins; an example is using $p_{\mathrm{T}}^{\gamma \gamma}$ to construct analysis categories targeting STXS bins defined by $p_{\mathrm{T}}^{\mathrm{H}}$ values. Increasing the total number of analysis categories to target individual STXS bins in this way does not degrade the analysis' sensitivity to the individual production mode and total Higgs boson cross sections. For each production mode, the event categorisation is designed to target all of the STXS bins to which some sensitivity can be obtained in the diphoton decay channel with the available data.

Several different machine learning (ML) algorithms are used throughout this analysis for both regression and classification tasks. Examples include regressions that improve the agreement between simulation and data, and classification to improve the discrimination between signal and background processes. The usage of ML techniques for event categorisation is also found to improve the separation between different STXS bins, which further improves the sensitivity of STXS measurements. For the training of boosted decision trees (BDTs), either the XGBOOst [35] or the TMVA package [36] package is used. The TensorFlow [37] package is used to train deep neural networks (DNNs).

For the ggH phase space, almost all of the STXS bins can be measured individually, without any bin merging (blue in figure 1$)$. The exceptions are the high dijet mass $\left(m_{\mathrm{jj}}\right)$ STXS bins, which are difficult to distinguish from VBF events. Furthermore, the sensitivity to STXS bins with particularly high $p_{\mathrm{T}}^{\mathrm{H}}$ is limited. Analysis categories are constructed using a BDT to assign the most probable STXS bin for each event. The amount of background is reduced using another BDT, referred to as the diphoton BDT. The diphoton BDT is trained to discriminate between all Higgs boson signal events and all other modes of SM diphoton 
production. Throughout the analysis, events originating from the $\mathrm{b} \overline{\mathrm{b}} \mathrm{H}$ production mode are grouped together with ggH events.

The VBF production mode and VH production where the vector boson decays hadronically are considered together as (EW) $\mathrm{qqH}$ production (orange in figure 1). A set of analysis categories enriched in VBF-like events, where a dijet with high $m_{\mathrm{jj}}$ is present, is defined. These analysis categories make use of the same diphoton BDT used in the analysis categories targeting $\mathrm{ggH}$ to reduce the number of background events. Additionally, a BDT based on the kinematic properties of the characteristic VBF dijet system, known as the dijet BDT, is utilised. The dijet BDT is trained to distinguish between three different classes of events with a VBF-like topology: VBF events, ggH events, and events produced by all other SM processes. This enables VBF events to be effectively separated from both VBF-like ggH events and other SM backgrounds. At least one analysis category is defined to target each VBF-like qqH STXS bin. Additional analysis categories enriched in VH-like events, where the vector boson decays hadronically to give a dijet whose $m_{\mathrm{jj}}$ is consistent with a $\mathrm{W}$ or $\mathrm{Z}$ boson, are defined. These make use of a dedicated $\mathrm{VH}$ hadronic BDT to reduce both the number of background events and contamination from $\mathrm{ggH}$ events.

Analysis categories targeting VH leptonic production (green in figure 1) are divided into three categorisation regions, containing either zero, one, or two reconstructed charged leptons (electrons or muons). Each categorisation region uses a dedicated BDT to reduce the background contamination. It is not possible to measure STXS bins individually with the available data set. Nonetheless, where a sufficient number of events exists, analysis categories are constructed to provide sensitivity to merged groups of STXS bins.

In this analysis, $t \bar{t} H$ and $t H$ production cross sections are measured independently ( $\mathrm{t} \overline{\mathrm{t} H}$ STXS bins are purple in figure 1, whilst tH is yellow). For this purpose, a dedicated DNN referred to as the top DNN is trained to discriminate between tH and $t \bar{t} \mathrm{H}$ events. An analysis category enriched in tH events is defined that uses the top DNN to reduce the contamination from $t \bar{t} \mathrm{H}$ events, with a BDT used to reject background events from other sources.

The analysis categories targeting t $t \bar{t} \mathrm{H}$ production are based on those described in ref. [24], with separate channels for hadronic and leptonic top quark decays. In each channel, a dedicated BDT is trained to reject background events. Furthermore, the top DNN is used to reduce the amount of contamination from tH events. The analysis categories are divided to provide the sensitivity to the STXS bins, for which four $p_{\mathrm{T}}^{\mathrm{H}}$ ranges are defined.

It is possible for an event to pass the selection criteria for more than one analysis category. To unambiguously assign each event to only one analysis category, a priority sequence is defined. Events that could enter more than one analysis category are assigned to the analysis category with the highest priority. The priority sequence is based on the expected number of signal events, with a higher priority assigned to analysis categories with a lower expected signal yield. This ordering enables the construction of analysis categories containing sufficiently high fractions of the Higgs boson production mechanisms with lower SM cross sections, which is necessary to perform independent measurements of these processes. 
Events in data and the corresponding simulation for all three years of data-taking from 2016 to 2018 are grouped together in the final analysis categories. This gives better performance than constructing analysis categories for each year individually, requiring fewer analysis categories in total for a comparable sensitivity. Separating the analysis categories by year would enable differences in the detector conditions - such as the variation in $m_{\gamma \gamma}$ resolution - to be exploited. However this is found to be less important than the advantage of having a greater number of events with which to train multivariate classifiers and optimise the analysis category definitions. Furthermore, the variations in detector conditions are relatively modest, and in general not substantially greater than variations within a given year of data-taking, which allows all data collected in each of the three years to be analysed together.

Nonetheless, simulated events are generated for each year separately, with the corresponding detector conditions, before they are merged together. This accounts for the variation in the detector itself, in the event reconstruction procedure, and in the LHC beam parameters. Furthermore, corrections to the photon energy scale and other procedures relating to the event reconstruction are also performed for each year individually. Only when performing the final division of selected diphoton events into the analysis categories are the simulated and data events from different years processed together. The full description of all the analysis categories is given in section 6 .

Once the selection criteria for each analysis category are defined, results are obtained by performing a simultaneous fit to the resulting $m_{\gamma \gamma}$ distributions in all analysis categories. The results of several different measurements with different observables are reported in section 9. For measurements within the STXS framework, it is not possible to measure each STXS bin individually. Therefore for each fit, a set of observables is defined by merging some STXS bins. In this paper, the results of two scenarios with different parameterisations of the STXS bins are provided. In addition, measurements of SM signal strength modifiers are reported, both for inclusive Higgs boson production and per production mode. Finally, measurements of Higgs boson couplings within the $\kappa$-framework are also shown.

\section{Data samples and simulated events}

The analysis exploits proton-proton collision data at $\sqrt{s}=13 \mathrm{TeV}$, collected in 2016, 2017, and 2018 and corresponding to integrated luminosities of $35.9,41.5$, and $59.4 \mathrm{fb}^{-1}$, respectively. The integrated luminosities of the 2016-2018 data-taking periods are individually known with uncertainties in the $2.3-2.5 \%$ range [38-40], while the total (2016-2018) integrated luminosity has an uncertainty of $1.8 \%$, the improvement in precision reflecting the (uncorrelated) time evolution of some systematic effects. In this section, the data sets and simulated event samples for all three years are described. Any differences between the years are highlighted in the text.

Events are selected using a diphoton high-level trigger with asymmetric photon $p_{\mathrm{T}}$ thresholds of 30 (30) and 18 (22) GeV in 2016 (2017 and 2018) data. A calorimetric selection is applied at trigger level, based on the shape of the electromagnetic shower, the isolation of the photon candidate, and the ratio of the hadronic and electromagnetic energy 
deposits of the shower. The $R_{9}$ variable is defined as the energy sum of the $3 \times 3$ crystals centred on the most energetic crystal in the candidate electromagnetic cluster divided by the energy of the candidate. The value of $R_{9}$ is used to identify photons undergoing a conversion in the material upstream of the ECAL. Unconverted photons typically have narrower transverse shower profiles, resulting in higher values of the $R_{9}$ variable, compared to converted photons. The trigger efficiency is measured from $\mathrm{Z} \rightarrow$ ee events using the "tag-and-probe" technique [41]. The efficiency measured in data in bins of $p_{\mathrm{T}}, R_{9}$, and $\eta$ is used to weight the simulated events to replicate the trigger efficiency observed in data.

A Monte Carlo (MC) simulated signal sample for each Higgs boson production mechanism is generated using MADGRAPH5_aMC@NLO (version 2.4.2) at next-to-leading order accuracy [42] in perturbative quantum chromodynamics (QCD). For each production mode, events are generated with $m_{\mathrm{H}}=120,125$, and $130 \mathrm{GeV}$. Events produced via the gluon fusion mechanism are weighted as a function of $p_{\mathrm{T}}^{\mathrm{H}}$ and the number of jets in the event, to match the prediction from the NNLOPS program [43]. All parton-level samples are interfaced with PYTHIA8 version 8.226 (8.230) [44] for parton showering and hadronization, with the CUETP8M1 [45] (CP5 [46]) tune used for the simulation of 2016 (2017 and 2018) data. Parton distribution functions (PDFs) are taken from the NNPDF 3.0 [47] (3.1 [48]) set, when simulating 2016 (2017 and 2018) data. The production cross sections and branching fractions recommended by the LHC Higgs Working Group [10] are used. The relative fraction of each STXS bin for each inclusive production mode at particle level is taken from simulation and used to compute the SM prediction for the production cross section in each STXS bin. Additional signal samples generated with POWHEG 2.0 [49-54] at nextto-leading order accuracy in perturbative QCD are used to train some of the multivariate discriminants described in section 6 .

The dominant source of background events in this analysis is due to SM diphoton production. A smaller component comes from $\gamma+$ jet or jet + jet events, in which jets are misidentified as photons. In the final fits of the analysis, the background is estimated directly from the diphoton mass distribution in data. Simulated background events from different event generators are only used for the training of multivariate discriminants. The diphoton background is generated with the SHERPA (version 2.2.4) generator [55]. It includes the Born processes with up to 3 additional jets, as well as the box processes at leading order accuracy. The $\gamma+$ jet and jet + jet backgrounds are simulated at leading order with PYTHIA8, after applying a filter at generator level to enrich the production of jets with a high electromagnetic activity. The filter requires a potential photon signal coming from photons, electrons, or neutral hadrons with $p_{\mathrm{T}}>15 \mathrm{GeV}$. In addition, the filter requires no more than two charged particles $\left(p_{\mathrm{T}}>1.6 \mathrm{GeV}\right.$ and $\left.|\eta|<2.2\right)$ in a cone of radius $R=\sqrt{(\Delta \eta)^{2}+(\Delta \varphi)^{2}}<0.2$ (where $\varphi$ is the azimuthal angle in radians) around the photon candidate, mimicking the tracker isolation described in section 5 .

A sample of Drell-Yan events is simulated with MADGRAPH5_amC@NLO, and is used both to derive corrections for simulation and for validation purposes.

The response of the CMS detector is simulated using the GEANT4 package [56]. This includes the simulation of the multiple proton-proton interactions taking place in each bunch crossing. These can occur at the nominal bunch crossing (in-time pileup) or at 
the crossing of previous and subsequent bunches (out-of-time pileup), and the simulation accounts for both. Simulated out-of-time pileup is limited to a window of $[-12,+3]$ bunch crossings around the nominal, in which the effects on the observables reconstructed in the detector are most relevant. Simulated events are weighted to reproduce the distribution of the number of interaction vertices in data. The average number of interactions per bunch crossing in data in the 2016 (2017 and 2018) data sets is 23 (32).

\section{Event reconstruction}

\subsection{Photon reconstruction}

Efficiently reconstructing photons with an accurate and precise energy determination plays a very important role in the sensitivity of this analysis. This section describes in detail the procedures used to reconstruct the photon energy and the photon preselection criteria.

Photon candidates are reconstructed from energy clusters in the ECAL not linked to any charged-particle trajectories seeded in the pixel detector. The clusters are built around a "seed" crystal, identified as a local energy maximum above a given threshold. The clusters are grown with a so-called topological clustering, where crystals with at least one side in common with a crystal already in the cluster and with an energy above a given threshold are added to the existing cluster itself. Finally, the clusters are dynamically merged into "superclusters" to ensure good containment of the shower, accounting for geometrical variation along $\eta$, and optimising the robustness of the energy resolution against pileup. The energy of the photon is estimated by summing the energy of each crystal in the supercluster, calibrated and corrected for response variations in time [57]. The photon energy is corrected for the imperfect containment of the electromagnetic shower and the energy losses from converted photons. The correction is computed with a multivariate regression technique trained on simulated photons, which estimates simultaneously the energy of the photon and its uncertainty.

After the application of this simulation-derived correction, some differences remain between data and simulation. A sequence of additional corrections are applied to improve the agreement between the two, using $\mathrm{Z} \rightarrow$ ee events where the electrons are reconstructed as photons. First, any residual drift in the energy scale in data over time is corrected for in bins corresponding approximately to the duration of one LHC fill. The second step involves modifying the energy scale in data and the energy resolution in simulation. A set of corrections is derived to align the mean of the dielectron mass spectrum in data with the expected value from simulation, and to smear the resolution in simulation to match that observed in data. These corrections are derived simultaneously in bins of $|\eta|$ and $R_{9}$. Further details on this procedure are contained in ref. [58].

Figure 2 shows comparisons between data and simulation after all corrections are applied for two cases where both electrons are reconstructed in the ECAL barrel and endcaps, respectively. In both cases the dielectron invariant mass spectra for the data and simulation are compatible within the uncertainties.

Once the photon energy correction has been applied, photon candidates are preselected before being used to form diphoton candidates. Requirements are placed on the photons' 

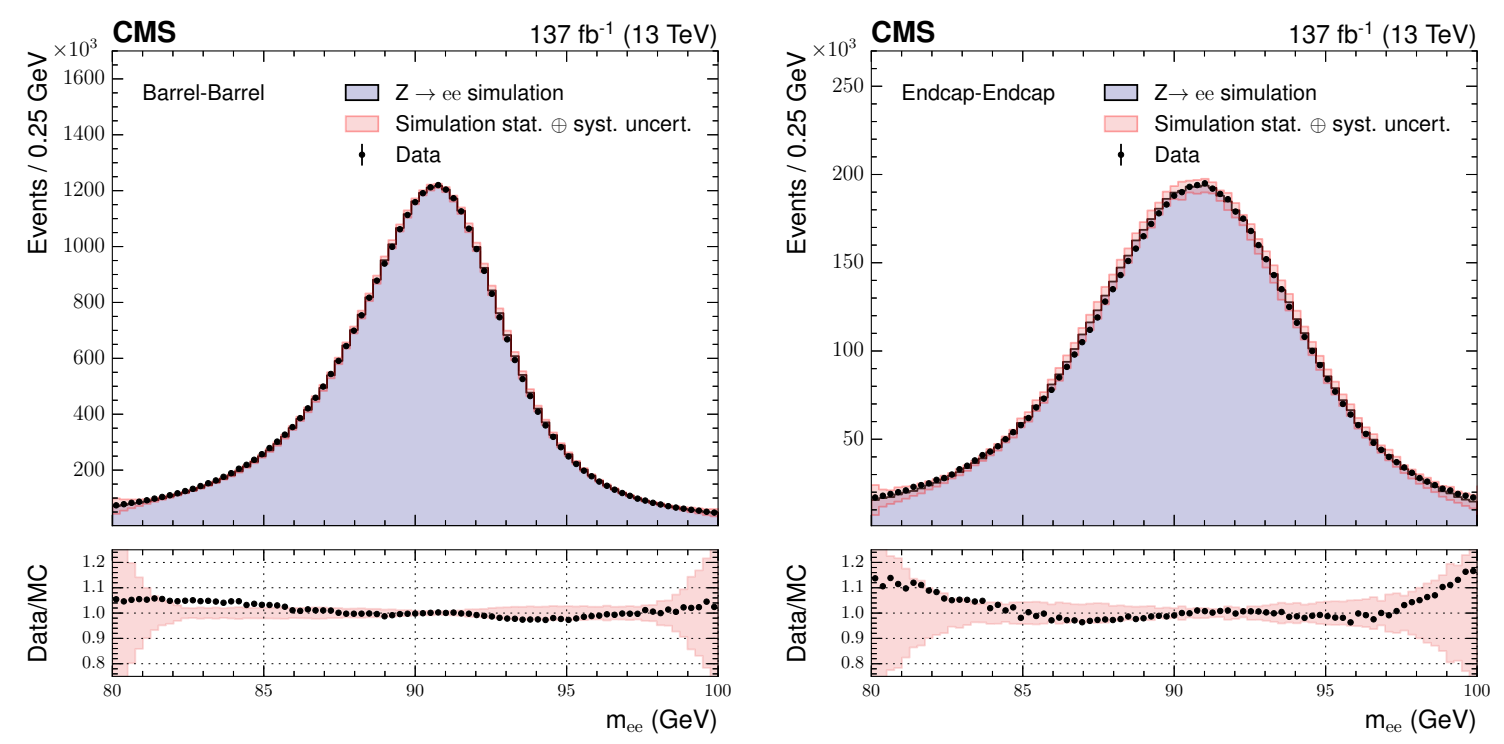

Figure 2. Comparison of the dielectron invariant mass spectra in data (black points) and simulation (blue histogram), after applying energy scale corrections to data and energy smearing to the simulation, for $\mathrm{Z} \rightarrow$ ee events with electrons reconstructed as photons. The statistical and systematic uncertainty on the simulation is shown by the pink band. The comparison is shown for events where both electrons are reconstructed in the ECAL barrel (left), and both in the ECAL endcaps (right). The lower panels show the ratio of the data to the MC simulation in black points, with the uncertainty on the ratio represented by the pink band. The full data set collected in 2016-2018 and the corresponding simulation are shown.

kinematic, shower shape, and isolation variables at values at least as stringent as those applied in the trigger. The preselection criteria are as follows:

- minimum $p_{\mathrm{T}}$ of the leading and subleading photons greater than 35 and $25 \mathrm{GeV}$, respectively;

- pseudorapidity of the photons $|\eta|<2.5$ and not in the barrel-endcap transition of $1.44<|\eta|<1.57$

- preselection on the $R_{9}$ variable and on $\sigma_{\eta \eta}$ - the lateral extension of the shower, defined as the energy-weighted spread within the $5 \times 5$ crystal matrix centred on the crystal with the largest energy deposit in the supercluster - to reject ECAL energy deposits incompatible with a single, isolated electromagnetic shower, such as those coming from neutral mesons;

- preselection on the ratio of the energy in the HCAL tower behind the supercluster's seed cluster to the energy in the supercluster $(\mathrm{H} / \mathrm{E})$, in order to reject hadronic showers;

- electron veto, which rejects the photon candidate if its supercluster in the ECAL is near to the extrapolated path of a track compatible with an electron. Tracks compatible with a reconstructed photon conversion vertex are not considered when applying this veto. 


\begin{tabular}{|cccccc|}
\hline \multirow{2}{*}{ Barrel } & $R_{9}$ & $\mathrm{H} / \mathrm{E}$ & $\sigma_{\eta \eta}$ & $\mathcal{I}_{\mathrm{ph}}(\mathrm{GeV})$ & $\mathcal{I}_{\mathrm{tk}}(\mathrm{GeV})$ \\
& {$[0.50,0.85]$} & $<0.08$ & $<0.015$ & $<4.0$ & $<6.0$ \\
& $>0.85$ & $<0.08$ & - & - & - \\
\multirow{2}{*}{ Endcaps } & {$[0.80,0.90]$} & $<0.08$ & $<0.035$ & $<4.0$ & $<6.0$ \\
& $>0.90$ & $<0.08$ & - & - & - \\
\hline
\end{tabular}

Table 1. Schema of the photon preselection requirements. The requirements depend both on whether a photon is in the barrel or endcap, and on its $R_{9}$ value.

- requirement on the photon isolation $\left(\mathcal{I}_{\mathrm{ph}}\right)$, defined as the $p_{\mathrm{T}}$ sum of the particles identified as photons inside a cone of size $R=0.3$ around the photon direction;

- requirement on the track isolation in a hollow cone $\left(\mathcal{I}_{\mathrm{tk}}\right)$, the $p_{\mathrm{T}}$ sum of all tracks in a cone of size $R=0.3$ around the photon candidate direction, excluding tracks in an inner cone of size $R=0.04$ to avoid counting tracks arising from photon conversion into electron-positron pairs;

- loose requirement on charged-hadron isolation $\left(\mathcal{I}_{\mathrm{ch}}\right)$, the $p_{\mathrm{T}}$ sum of charged hadrons inside a cone of size $R=0.3$ around the photon candidate.

The geometrical acceptance requirement is applied to the supercluster position in the ECAL. The requirement on the photon $p_{\mathrm{T}}$ is applied after the vertex assignment, which is described in further detail in section 5.3. The preselection thresholds are shown in table 1. Additionally, photons are required to satisfy at least one of $R_{9}>0.8, \mathcal{I}_{\mathrm{ch}} / p_{\mathrm{T}}^{\gamma}<0.3$, and $\mathcal{I}_{\text {ch }}<20 \mathrm{GeV}$.

The preselection efficiency is measured with the tag-and-probe technique using $\mathrm{Z} \rightarrow$ ee events in data, while the efficiency of the electron veto is measured in $Z \rightarrow \mu \mu \gamma$ events in data.

\subsection{Photon identification}

Photons in events passing the preselection criteria are further required to satisfy a photon identification criterion based on a BDT trained to separate genuine ("prompt") photons from jets mimicking a photon signature. This ID BDT is trained on a simulated sample of $\gamma+$ jet events, where prompt photons are used as the signal, while jets are used as the background. Input variables to the ID BDT include shower shape variables, isolation variables, the photon energy and $\eta$, and global event variables sensitive to pileup, such as the median energy density per unit area $\rho[12]$.

Simulated inputs for the photon ID BDT, both shower shape and isolation variables, are corrected to agree with data using a chained quantile regression (CQR) method [59]. This method was developed to improve the agreement in the photon ID BDT output between data and simulation, thus reducing the size of the associated systematic uncertainty relative to previous analyses. Corrections are derived using an unbiased set of electrons from $\mathrm{Z} \rightarrow$ ee events selected with a tag-and-probe method. The CQR comprises a set of BDTs that predict the cumulative distribution function $(\mathrm{CDF})$ of a given input variable. Its 
prediction is conditional upon three electron kinematic variables $\left(p_{\mathrm{T}},|\eta|, \phi\right)$ and $\rho$. The CDFs extracted in this way from data and simulated events are then used to derive a correction factor to be applied to any given simulated electron. These correction factors morph the CDF of the simulated shower shape onto the one observed in data.

The CQR method accounts for correlations among the shower shape variables and adjusts the correlation in the simulation to match the one observed in data. To achieve this, an ordered chain of the shower shape variables is constructed. The CDF of the first shower shape variable is predicted solely from the electron kinematic variables and event $\rho$ values, while the corrected values of the previously processed shower shape variables are also added as inputs for subsequent predictions. The order of the different shower shape variables in the chain is optimised to minimise the final discrepancy of the ID BDT score between data and simulation.

The isolation variables are not included in the chain since their correlation with the shower shape variables is negligible. Furthermore, there is a $p_{\mathrm{T}}$ threshold on the particle candidates included in the computation of the isolation variables. This causes these variables to follow a disjoint distribution, with a gap present between the peak at zero and a tail at positive values. The CDF of the isolation variables are therefore constant over the range of values between zero and the start of the tail, which prevents the use of the same technique used for the shower shape variables. The CQR method is thus extended with additional BDTs that are used to match, again based on the electron kinematic variables and the event $\rho$ value, the relative population of the peak and tail between data and simulation. The tails of the isolation variable distributions themselves are then morphed using the same technique for the shower shape variables.

A systematic uncertainty associated with the corrections is also included in the analysis. This is estimated by rederiving the corrections with equally sized subsets of the $\mathrm{Z} \rightarrow$ ee events used for training. Its magnitude corresponds to the standard deviation of the eventby-event differences in the corrected ID BDT output score obtained with the two training subsets. This uncertainty reflects the limited capacity of the network arising from the finite size of the training set. The size of the resulting experimental uncertainty is smaller than that required to cover discrepancies between data and simulation in previous versions of this analysis.

The distribution of the photon ID BDT for the lowest scoring photon for signal events and the different background components is shown in figure 3, together with a comparison of data and simulation using $\mathrm{Z} \rightarrow$ ee events where the electrons are reconstructed as photons. These $\mathrm{Z} \rightarrow$ ee events are chosen because of the similarity in the detector signature and reconstruction procedures for electrons and photons. Here, the electrons being reconstructed as photons means that the track information is not used, and the energy is determined using the algorithm and corrections corresponding to photons rather than electrons. The photon ID BDT distribution is also checked with photons using $\mathrm{Z} \rightarrow \mu \mu \gamma$ events, where data and simulation are found to agree within uncertainties.

As an additional preselection criterion, photons are required to have a photon identification BDT score of at least -0.9 . Both photons pass this additional requirement in more than $99 \%$ of simulated signal events. The efficiency of the requirement in simulation 

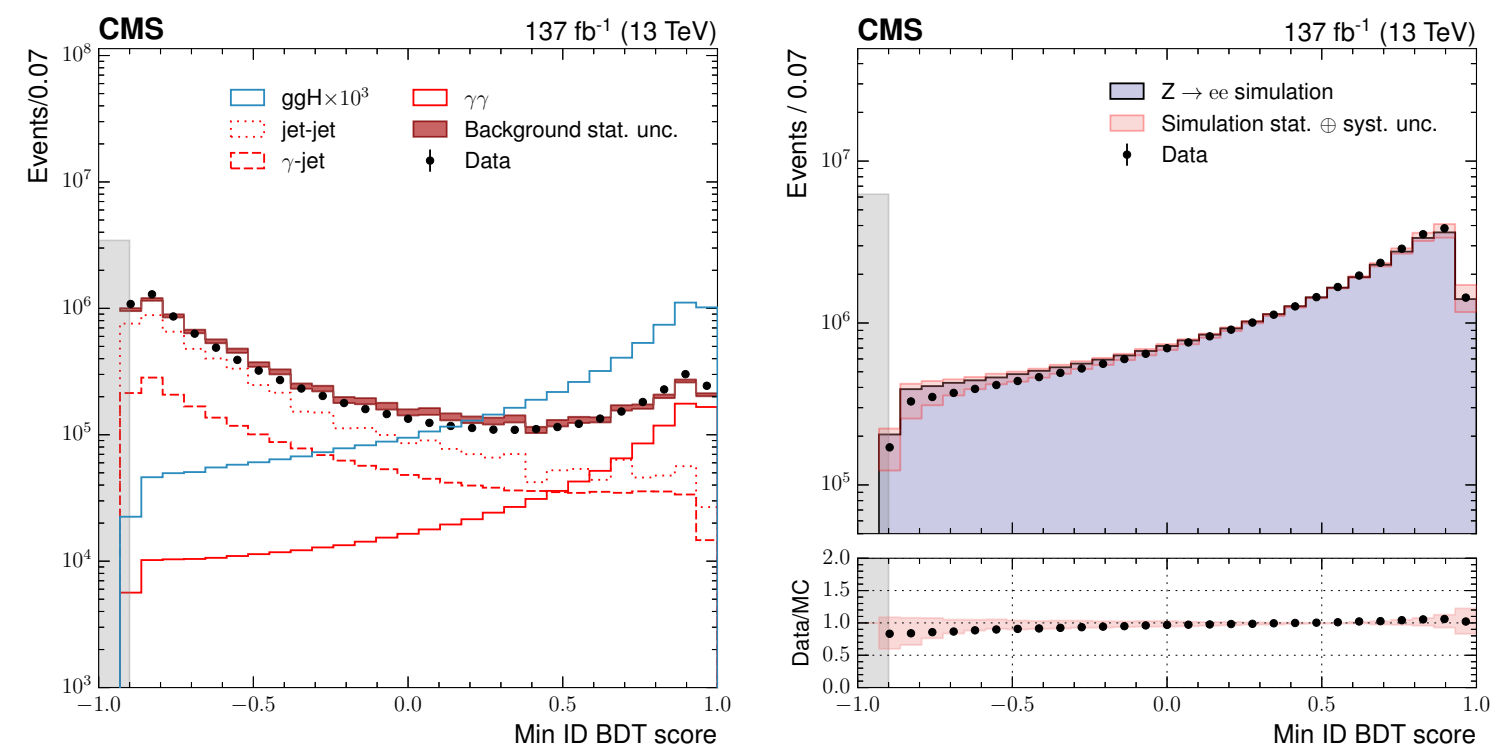

Figure 3. The left plot shows the distribution of the photon identification BDT score of the lowest scoring photon in diphoton pairs with $100<m_{\gamma \gamma}<180 \mathrm{GeV}$, for data events passing the preselection (black points), and for simulated background events (red band). Histograms are also shown for different components of the simulated background. The blue histogram corresponds to simulated Higgs boson signal events. The right plot shows the same distribution for $\mathrm{Z} \rightarrow$ ee events in data and simulation, where the electrons are reconstructed as photons. The statistical and systematic uncertainty in simulation is also shown (pink band). Photons with an identification BDT score in the grey shaded region (below -0.9) are not considered in the analysis. The full data set collected in 2016-2018 and the corresponding simulation are shown.

is corrected to match that in data using $\mathrm{Z} \rightarrow$ ee events, and a corresponding systematic uncertainty is introduced.

\subsection{Diphoton vertex identification}

The determination of the primary vertex from which the two photons originate has a direct impact on the $m_{\gamma \gamma}$ resolution. If the position along the beam axis $(z)$ of the interaction producing the diphoton is known to better than around $1 \mathrm{~cm}$, the $m_{\gamma \gamma}$ resolution is dominated by the photon energy resolution.

The RMS of the distribution in $z$ of the reconstructed vertices in data in 2016-2018 varies in the range $3.4-3.6 \mathrm{~cm}$. The corresponding distribution in each year's simulation is reweighted to match that in data.

The diphoton vertex assignment is performed using a BDT (the vertex identification $\mathrm{BDT}$ ) whose inputs are observables related to tracks recoiling against the diphoton system [12]. It is trained on simulated $\mathrm{ggH}$ events and identifies a single vertex in each event.

The performance of the vertex identification BDT is validated using $\mathrm{Z} \rightarrow \mu^{+} \mu^{-}$events. The vertices are refitted with the muon tracks omitted from the fit, to mimic a diphoton system. Figure 4 (left plot) shows the efficiency of correctly assigning the vertex, as a 

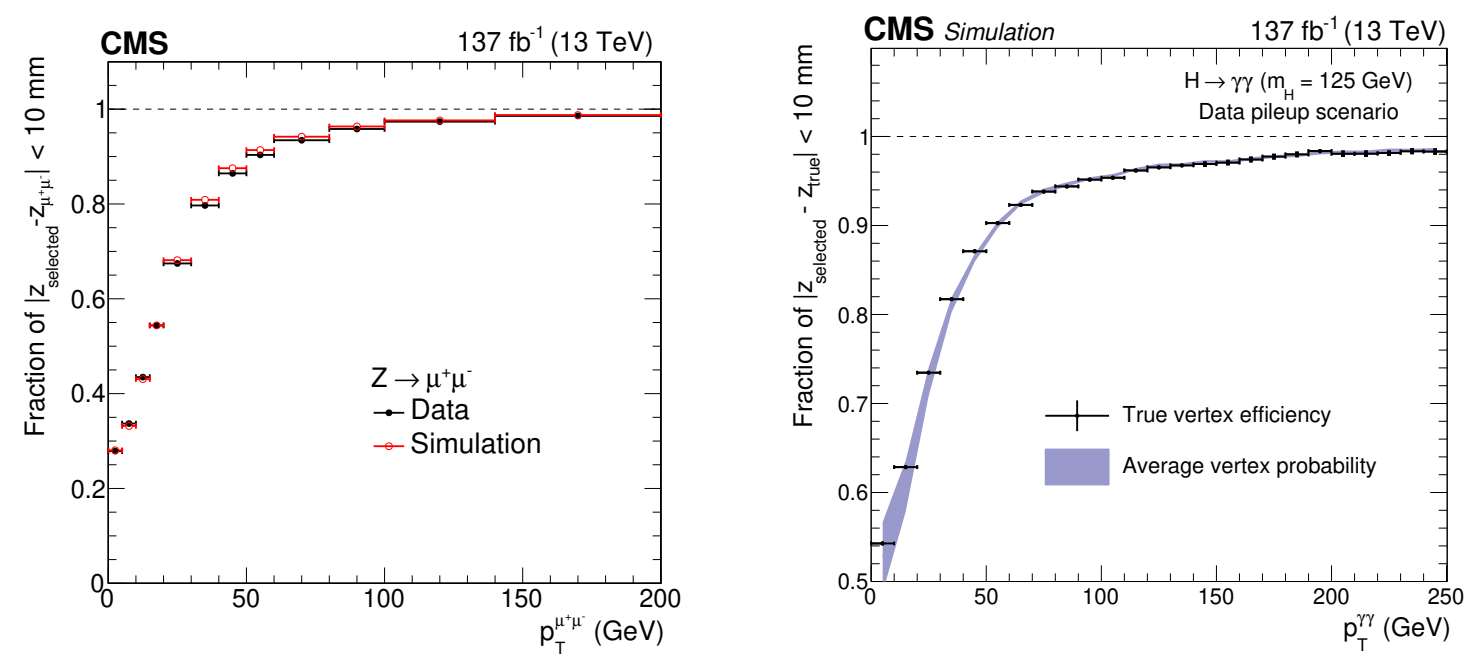

Figure 4. The left plot shows the validation of the $\mathrm{H} \rightarrow \gamma \gamma$ vertex identification algorithm on $\mathrm{Z} \rightarrow \mu^{+} \mu^{-}$events, where the muon tracks are omitted when performing the event reconstruction. This allows the fraction of events with the correctly assigned vertex estimated with simulation to be compared with data, as a function of the $p_{\mathrm{T}}$ of the dimuon system, serving as a validation of the vertex identification BDT. Simulated events are weighted to match the distributions of pileup and distribution of vertices along the beam axis in data. The right plot demonstrates that the average vertex probability to be within $1 \mathrm{~cm}$ of the true vertex agrees with the true vertex efficiency in simulated events. The full data set collected in 2016-2018 and the corresponding simulation are shown.

function of the dimuon $p_{\mathrm{T}}$. The data and simulation agree to within approximately $2 \%$ across the entire $p_{\mathrm{T}}$ range. Nonetheless, the simulation is subsequently corrected to match the efficiencies measured in data, whilst preserving the total number of events. A systematic uncertainty is introduced with a magnitude equal to the size of this correction.

The efficiency of assigning the diphoton vertex to be within $1 \mathrm{~cm}$ of the true vertex in simulated $\mathrm{H} \rightarrow \gamma \gamma$ events is approximately $79 \%$. The events with an incorrectly-assigned vertex are primarily $\mathrm{ggH}$ events with zero additional jets, and the associated systematic uncertainty affects ggH events only.

A second vertex-related multivariate discriminant, the vertex probability BDT, estimates the probability that the vertex, chosen by the vertex identification BDT, is within $1 \mathrm{~cm}$ of the vertex from which the diphoton originated. The vertex probability BDT is trained on simulated $\mathrm{H} \rightarrow \gamma \gamma$ events using input variables relating to the vertices in the event, their vertex identification BDT scores, the number of photons with associated conversion tracks, and the $p_{\mathrm{T}}$ of the diphoton system. Agreement is observed between the average vertex probability and the vertex efficiency in simulation, as shown in figure 4 (right plot).

\subsection{Additional objects}

Objects in the event other than the two photons are reconstructed as described in section 2. Charged hadrons originating from interaction vertices other than the one chosen 
by the vertex identification BDT are removed from the analysis. In addition, all jets are required to have $p_{\mathrm{T}}>25 \mathrm{GeV}$, be within $|\eta|<4.7$, and be separated from both photons by $\Delta R($ jet, $\gamma)>0.4$. Depending on the analysis category, more stringent constraints on the jet $p_{\mathrm{T}}$ and $|\eta|$ may be imposed; this is described in the text where relevant. In addition, some analysis categories require that jets also pass an identification criterion designed to reduce the number of selected jets originating from pileup collisions [60]. Jets from the hadronization of bottom quarks are tagged using a DNN that takes secondary vertices and PF candidates as inputs [61].

Electrons and muons are used in the analysis categories targeting t $\overline{\mathrm{t}} \mathrm{H}$ and leptonic $\mathrm{VH}$ production. Electrons are required to have $p_{\mathrm{T}}>10 \mathrm{GeV}$ and be within $|\eta|<2.4$, excluding the barrel-endcap transition region. Muons must have $p_{\mathrm{T}}>5 \mathrm{GeV}$ and fall within $|\eta|<2.4$. In addition, isolation and identification requirements are imposed on both [62, 63].

\section{Event categorisation}

The event selection in all analysis categories requires the two leading preselected photon candidates to have $p_{\mathrm{T}}^{\gamma 1}>m_{\gamma \gamma} / 3$ and $p_{\mathrm{T}}^{\gamma 2}>m_{\gamma \gamma} / 4$, respectively, with an invariant mass in the range $100<m_{\gamma \gamma}<180 \mathrm{GeV}$. The requirements on the scaled photon $p_{\mathrm{T}}$ prevent distortions at the lower end of the $m_{\gamma \gamma}$ spectrum. As described in section 3, events are divided into analysis categories to provide sensitivity to different production mechanisms and STXS bins. Each analysis category is designed to select as many events as possible from a given STXS bin, or set of bins, referred to here as the target bin or bins. The requirements for each analysis category should also select as few events from other, nontargeted STXS bins as possible, to enable simultaneous measurements of different cross sections. Finally, the selection should also reject as many background events as possible, to maximise the measurements' eventual sensitivity. This section describes the several different categorisation schemes used for different event topologies, and the relevant STXS bins for each.

The STXS bins themselves are defined using particle-level quantities. In all targeted bins, $\left|y_{\mathrm{H}}\right|$ is required to be less than 2.5. Jets are clustered using the anti- $k_{\mathrm{T}}$ algorithm [19] with a distance parameter of 0.4. All stable particles, except for those arising from the decay of the Higgs boson or the leptonic decay of an associated vector boson, are included in the clustering. Jets are also required to have $p_{\mathrm{T}}>30 \mathrm{GeV}$. The definition of leptons includes electrons, muons, and tau leptons. Further details of the objects used to define the STXS bins can be found in ref. [10].

In many of the categorisation schemes, ML algorithms are used to classify signal events or discriminate between signal and background processes. The output scores of the algorithms can then form part of the selection criteria used to define analysis categories. Where these ML techniques are used to classify events, two types of validation are performed. Firstly, in the typical case where simulated signal and background events are used to train the algorithm, a comparison of the simulated background to the corresponding data is performed. Good agreement between the two gives confidence that the background processes are accurately modelled and therefore that the ML algorithm performs well in 
its classification task. Since the background model used in the final maximum likelihood fit is derived directly from data, poor agreement in background-like regions cannot induce any biases, but only result in sub-optimal performance of the classifier. The second form of validation involves finding a signal-like region in which to compare the classifier output scores in simulation and data. Here the aim of the comparison is to instil confidence that simulated Higgs boson signal events, which do enter the final measurement, are sufficiently well-modelled. Therefore simulation and data should be expected to agree within statistical and systematic uncertainties in these cases. Furthermore, for all of the classifiers, the input variables are chosen such that $m_{\gamma \gamma}$ cannot be inferred. This prevents distortion of the $m_{\gamma \gamma}$ spectrum when applying selection thresholds on the output scores.

A summary of all the analysis categories, together with the STXS bin or bins each analysis category targets, is given in section 6.6.

\subsection{Event categories for ggH production}

The definitions of the ggH STXS bins are given in table 2, corresponding to the blue entries in figure 1. The bins are defined using $p_{\mathrm{T}}^{\mathrm{H}}$, the number of jets, and $m_{\mathrm{jj}}$. Those bins with $p_{\mathrm{T}}^{\mathrm{H}}>200 \mathrm{GeV}$ are referred to as "BSM" bins because they have a cross section that is predicted to be low in the SM, but which could be enhanced by the presence of additional BSM particles. Events originating from ggZH production in which the $\mathrm{Z}$ boson decays hadronically are included in the definition of $\mathrm{ggH}$. Analysis categories are defined to target each ggH STXS bin independently, except for those in the VBF-like phase space. Events from the VBF-like bins are categorised separately, as described in section 6.2.

The $\mathrm{ggH}$ categorisation procedure can be summarised as follows. First, events are classified using the so-called ggH BDT. The ggH BDT predicts the probability that a diphoton event belongs to a given ggH STXS class. Each class corresponds either to an individual STXS bin or to a set of multiple STXS bins. The first eight classes considered by the ggH BDT are individual STXS bins. These comprise the zero, one, and two jet bins with $p_{\mathrm{T}}^{\mathrm{H}}<200 \mathrm{GeV}$ and $m_{\mathrm{jj}}<350 \mathrm{GeV}$, corresponding to the eight leftmost ggH STXS bins in figure 1. To minimise model-dependence, the $\mathrm{ggH}$ BDT is not trained to distinguish between the STXS bins with $p_{\mathrm{T}}^{\mathrm{H}}>200 \mathrm{GeV}$. Instead, all events with $p_{\mathrm{T}}^{\mathrm{H}}>200 \mathrm{GeV}$ are treated as a single class, consisting of a set of four STXS bins. Hence, the task of the ggH BDT amounts to predicting one of nine ggH classes, which are uniquely defined by $p_{\mathrm{T}}^{\mathrm{H}}$ and the number of jets. Each event is then assigned to an analysis category based upon its most probable STXS bin, as determined by the $\mathrm{ggH}$ BDT. Events for which the maximum probability corresponds to the $p_{\mathrm{T}}^{\mathrm{H}}>200 \mathrm{GeV}$ class are assigned into an analysis category targeting one of the four STXS bins with $p_{\mathrm{T}}^{\mathrm{H}}>200 \mathrm{GeV}$. This assignment is performed using the event's reconstructed $p_{\mathrm{T}}^{\gamma \gamma}$ value. Finally, the analysis' sensitivity is maximised by further dividing the analysis categories using the diphoton BDT, which is trained to discriminate between signal and background processes and described in further detail below.

The $\mathrm{ggH}$ BDT is trained using simulated $\mathrm{ggH}$ events only. Input features to the $\mathrm{ggH}$ BDT are properties of the photons and quantities related to the kinematic properties of up to three $p_{\mathrm{T}}>20 \mathrm{GeV}$ jets. The photon features used are the photon kinematic variables, 


\begin{tabular}{|c|c|c|c|c|}
\hline \multirow{2}{*}{ STXS bin } & \multirow{2}{*}{$\begin{array}{c}\text { Definition } \\
\text { units of } p_{\mathrm{T}}^{\mathrm{H}}, m_{\mathrm{jj}} \text { and } p_{\mathrm{T}}^{\mathrm{Hjj}} \text { in } \mathrm{GeV}\end{array}$} & \multicolumn{2}{|c|}{ Fraction of cross section } & \multirow{2}{*}{$\sigma_{\mathrm{SM}} \mathcal{B}(\mathrm{fb})$} \\
\hline & & $\operatorname{ggH}$ & $\mathrm{gg} \rightarrow \mathrm{Z}(\mathrm{q} \overline{\mathrm{q}}) \mathrm{H}$ & \\
\hline $\operatorname{ggH}$ forward & $\left|y_{\mathrm{H}}\right|>2.5$ & $8.09 \%$ & $2.73 \%$ & 8.93 \\
\hline $\operatorname{ggH} 0 \mathrm{~J}$ low $p_{\mathrm{T}}^{\mathrm{H}}$ & Exactly 0 jets, $p_{\mathrm{T}}^{\mathrm{H}}<10$ & $13.87 \%$ & $0.01 \%$ & 15.30 \\
\hline $\operatorname{ggH} 0 \mathrm{~J}$ high $p_{\mathrm{T}}^{\mathrm{H}}$ & Exactly 0 jets, $10<p_{\mathrm{T}}^{\mathrm{H}}<200$ & $39.40 \%$ & $0.29 \%$ & 43.45 \\
\hline $\operatorname{ggH} 1 \mathrm{~J}$ low $p_{\mathrm{T}}^{\mathrm{H}}$ & Exactly 1 jet, $p_{\mathrm{T}}^{\mathrm{H}}<60$ & $14.77 \%$ & $2.00 \%$ & 16.29 \\
\hline $\operatorname{ggH} 1 \mathrm{~J}$ med $p_{\mathrm{T}}^{\mathrm{H}}$ & Exactly 1 jet, $60<p_{\mathrm{T}}^{\mathrm{H}}<120$ & $10.23 \%$ & $5.34 \%$ & 11.29 \\
\hline $\operatorname{ggH} 1 \mathrm{~J} \operatorname{high} p_{\mathrm{T}}^{\mathrm{H}}$ & Exactly 1 jet, $120<p_{\mathrm{T}}^{\mathrm{H}}<200$ & $1.82 \%$ & $3.53 \%$ & 2.01 \\
\hline $\operatorname{ggH} \geq 2 \mathrm{~J}$ low $p_{\mathrm{T}}^{\mathrm{H}}$ & At least 2 jets, $p_{\mathrm{T}}^{\mathrm{H}}<60, m_{\mathrm{jj}}<350$ & $2.56 \%$ & $5.74 \%$ & 2.83 \\
\hline $\operatorname{ggH} \geq 2 \mathrm{~J} \operatorname{med} p_{\mathrm{T}}^{\mathrm{H}}$ & At least 2 jets, $60<p_{\mathrm{T}}^{\mathrm{H}}<120, m_{\mathrm{jj}}<350$ & $4.10 \%$ & $19.63 \%$ & 4.56 \\
\hline $\operatorname{ggH} \geq 2 \mathrm{~J}$ high $p_{\mathrm{T}}^{\mathrm{H}}$ & At least 2 jets, $120<p_{\mathrm{T}}^{\mathrm{H}}<200, m_{\mathrm{jj}}<350$ & $1.88 \%$ & $29.55 \%$ & 2.13 \\
\hline $\operatorname{ggH}$ BSM $200<p_{\mathrm{T}}^{\mathrm{H}}<300$ & No jet requirements, $200<p_{\mathrm{T}}^{\mathrm{H}}<300$ & $0.98 \%$ & $13.93 \%$ & 1.11 \\
\hline $\operatorname{ggH}$ BSM $300<p_{\mathrm{T}}^{\mathrm{H}}<450$ & No jet requirements, $300<p_{\mathrm{T}}^{\mathrm{H}}<450$ & $0.25 \%$ & $3.86 \%$ & 0.28 \\
\hline $\operatorname{ggH} \mathrm{BSM} 450<p_{\mathrm{T}}^{\mathrm{H}}<650$ & No jet requirements, $450<p_{\mathrm{T}}^{\mathrm{H}}<650$ & $0.03 \%$ & $0.77 \%$ & 0.03 \\
\hline $\operatorname{ggH} \operatorname{BSM} p_{\mathrm{T}}^{\mathrm{H}}>650$ & No jet requirements, $p_{\mathrm{T}}^{\mathrm{H}}>650$ & $0.01 \%$ & $0.20 \%$ & 0.01 \\
\hline ggH VBF-like low $m_{\mathrm{jj}}$ low $p_{\mathrm{T}}^{\mathrm{Hjj}}$ & $\begin{array}{l}\text { At least } 2 \text { jets, } p_{\mathrm{T}}^{\mathrm{H}}<200, \\
350<m_{\mathrm{jj}}<700, p_{\mathrm{T}}^{\mathrm{Hjj}}<25\end{array}$ & $0.63 \%$ & $1.14 \%$ & 0.70 \\
\hline $\operatorname{ggH}$ VBF-like low $m_{\mathrm{jj}}$ high $p_{\mathrm{T}}^{\mathrm{H} j \mathrm{j}}$ & $\begin{array}{l}\text { At least } 2 \text { jets, } p_{\mathrm{T}}^{\mathrm{H}}<200, \\
350<m_{\mathrm{jj}}<700, p_{\mathrm{T}}^{\mathrm{H} j \mathrm{j}}>25\end{array}$ & $0.77 \%$ & $8.06 \%$ & 0.86 \\
\hline ggH VBF-like high $m_{\mathrm{jj}}$ low $p_{\mathrm{T}}^{\mathrm{H} j \mathrm{j}}$ & $\begin{array}{l}\text { At least } 2 \text { jets, } p_{\mathrm{T}}^{\mathrm{H}}<200 \\
m_{\mathrm{jj}}>700, p_{\mathrm{T}}^{\mathrm{Hjj}}<25\end{array}$ & $0.28 \%$ & $0.36 \%$ & 0.31 \\
\hline $\operatorname{ggH}$ VBF-like high $m_{\mathrm{jj}}$ high $p_{\mathrm{T}}^{\mathrm{Hjj}}$ & $\begin{array}{c}\text { At least } 2 \text { jets, } p_{\mathrm{T}}^{\mathrm{H}}<200 \\
m_{\mathrm{jj}}>700, p_{\mathrm{T}}^{\mathrm{H} j \mathrm{j}}>25\end{array}$ & $0.32 \%$ & $2.85 \%$ & 0.36 \\
\hline
\end{tabular}

Table 2. Definition of the ggH STXS bins. The product of the cross section and branching fraction $(\mathcal{B})$, evaluated at $\sqrt{s}=13 \mathrm{TeV}$ and $m_{\mathrm{H}}=125 \mathrm{GeV}$, is given for each bin in the last column. The fraction of the total production mode cross section from each STXS bin is also shown. Events originating from ggZH production, in which the $\mathrm{Z}$ decays hadronically, are grouped together with the corresponding ggH STXS bin in the STXS measurements and are shown as a separate column in the table. The $\mathrm{b} \bar{b} \mathrm{H}$ production mode, whose $\sigma_{\mathrm{SM}} \mathcal{B}=1.054 \mathrm{fb}$, is grouped together with the $\operatorname{ggH}$ 0J high $p_{\mathrm{T}}^{\mathrm{H}}$ bin. Unless stated otherwise, the STXS bins are defined for $\left|y_{\mathrm{H}}\right|<2.5$. Events with $\left|y_{\mathrm{H}}\right|>2.5$ are mostly outside of the experimental acceptance and therefore have a negligible contribution to all analysis categories.

ID BDT scores, $m_{\gamma \gamma}$ resolution estimates, and the vertex probability estimate. The $p_{\mathrm{T}}^{\gamma \gamma}$ value is also included as an input. As previously mentioned, the set of variables is chosen such that $m_{\gamma \gamma}$ cannot be inferred from the inputs; for this reason, the $p_{\mathrm{T}} / m_{\gamma \gamma}$ values of each photon, rather than $p_{\mathrm{T}}$, are used. The variables related to jets include the kinematic variables and pileup ID scores of the three leading jets in the event.

The resulting STXS bin assignment performs better than simply using the reconstructed $p_{\mathrm{T}}^{\gamma \gamma}$ and number of jets. The fraction of selected ggH events in simulation that are assigned to the correct STXS bin increases from 77 to $82 \%$ when using the ggH BDT rather than the reconstructed $p_{\mathrm{T}}^{\gamma \gamma}$ and number of jets. This improvement can be explained by the fact that the ggH BDT is able to exploit the correlations between the photon and jet kinematic properties. In this way, the well-measured photon quantities can be used 


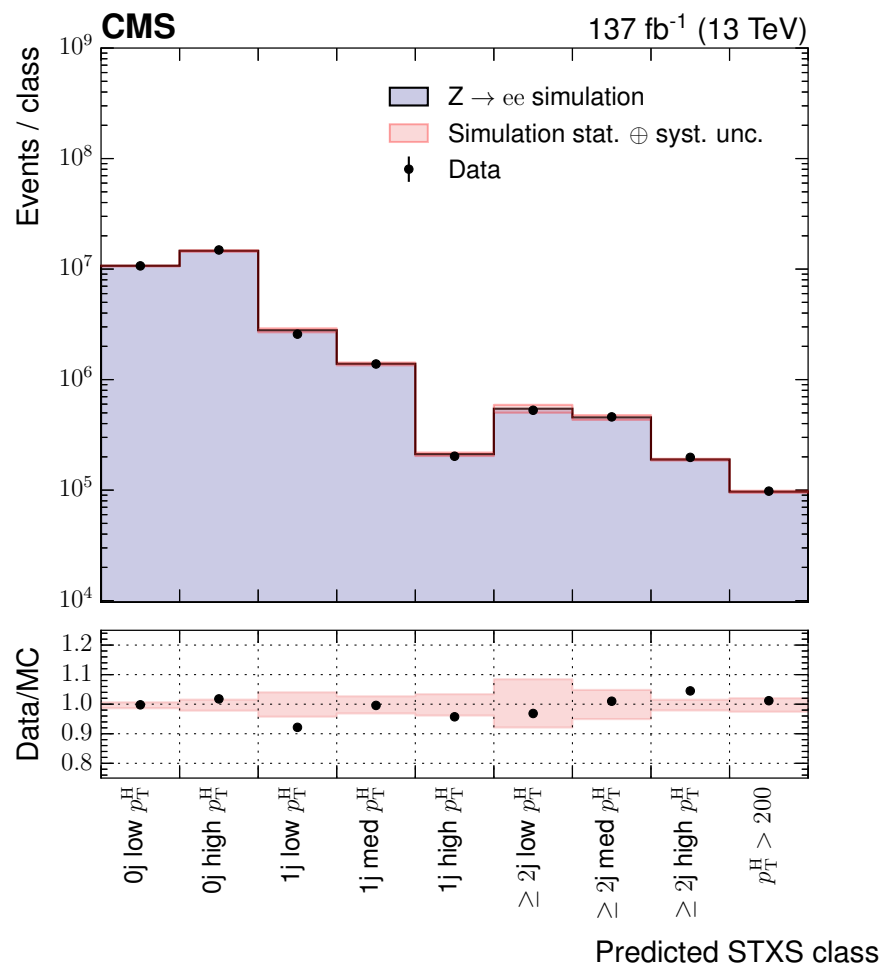

Figure 5. The most probable STXS class from the ggH BDT in $\mathrm{Z} \rightarrow$ ee events where the electrons are reconstructed as photons is shown. The points show the predicted class for data, whilst the histogram shows predicted score for simulated Drell-Yan events, including statistical and systematic uncertainties (pink band). The full data set collected in 2016-2018 and the corresponding simulation are shown.

to infer information about the less well-measured jets. As a result, the contamination of analysis categories due to migration across jet bins is reduced; the migration across $p_{\mathrm{T}}^{\gamma \gamma}$ boundaries is much smaller and essentially unchanged by the $\mathrm{ggH}$ BDT. The $\mathrm{ggH}$ BDT therefore slightly improves the analysis sensitivity, most noticeably in the zero- and one-jet bins. Furthermore, the correlations between cross section parameters in the final fits are reduced.

To validate the modelling of the $\mathrm{ggH}$ BDT and its input variables, the agreement in the STXS class prediction between data and simulation in $\mathrm{Z} \rightarrow$ ee events, with electrons reconstructed as photons, is checked. Figure 5 shows the number of events predicted to belong to each event class. The uncertainties in the photon ID BDT, the photon energy resolution, and the jet energy scale and resolution are included. There is good agreement between data and simulation in this signal-like region.

The diphoton BDT is used, after events are classified by the ggH BDT, to reduce the background from SM diphoton production, thereby maximising the analysis sensitivity. The diphoton BDT is trained with all Higgs boson signal events against SM diphoton production as background. A high score is assigned to events with photons showing signallike kinematic properties, good $m_{\gamma \gamma}$ resolution, and high photon identification BDT score. 

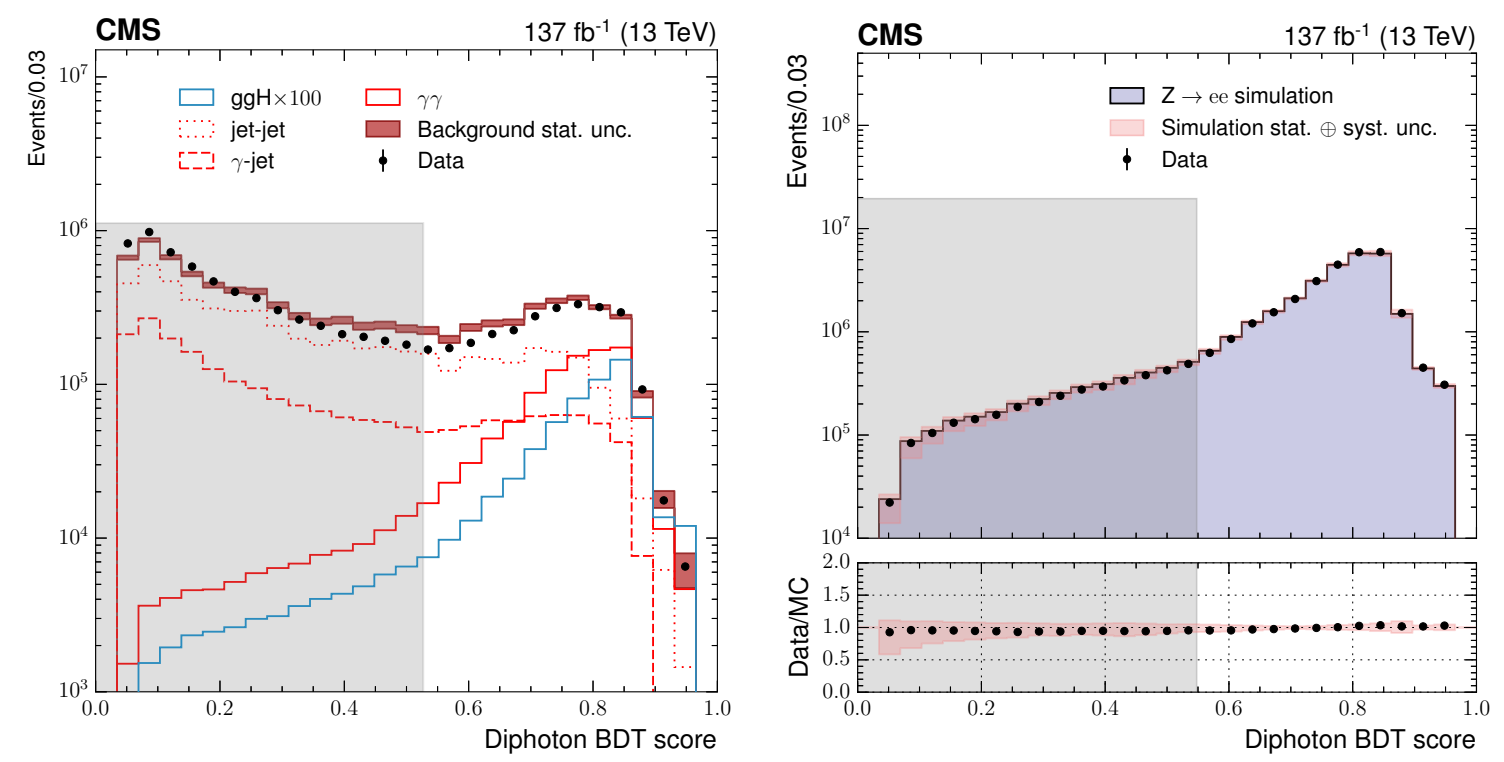

Figure 6. The left plot shows the distribution of the diphoton BDT score in events with $m_{\gamma \gamma}$ in the range 100-120 or $130-180 \mathrm{GeV}$, for data events passing the preselection (black points), and for simulated background events (red band). Histograms are also shown for different components of the simulated background in red. The blue histogram corresponds to simulated Higgs boson signal events $(\times 100)$. The right plot shows the same distribution in $\mathrm{Z} \rightarrow$ ee events where the electrons are reconstructed as photons. The points show the score for data, the histogram shows the score for simulated Drell-Yan events, including statistical and systematic uncertainties (pink band). The regions shaded grey contain diphoton BDT scores below the lowest threshold used to define an analysis category. The full data set collected in 2016-2018 and the corresponding simulation are shown.

The input variables to the classifier are the photon kinematic variables, ID BDT scores, $m_{\gamma \gamma}$ resolution estimates and the vertex probability estimate.

Figure 6 shows the output score of the diphoton BDT for signal and background events, together with corresponding data from the $m_{\gamma \gamma}$ sidebands, meaning $100<m_{\gamma \gamma}<120 \mathrm{GeV}$ or $130<m_{\gamma \gamma}<180 \mathrm{GeV}$. A validation of the diphoton BDT obtained in $\mathrm{Z} \rightarrow$ ee events, where the electrons are reconstructed as photons, is also shown in figure 6. Here the data and simulation agree within the statistical and systematic uncertainties.

After being classified by the ggH BDT, events are divided into analysis categories using the diphoton BDT, with the boundaries chosen to maximise the expected sensitivity. The resulting analysis categories are referred to as "tags". For ggH production, there is at least one tag targeting each individual STXS bin, except for the VBF-like bins. The tag names are given in decreasing order of the expected ratio of signal-to-background events (S/B). For example, the tag with the highest S/B targeting the $\mathrm{ggH}$ zero jet bin with $p_{\mathrm{T}}^{\mathrm{H}}<10 \mathrm{GeV}$ is denoted 0J low $p_{\mathrm{T}}^{\gamma \gamma}$ Tag 0 .

The expected signal and background yields in each ggH analysis category are shown in table 3. The yields shown in this and subsequent tables correspond to those in the final analysis categories, meaning that events selected by analysis categories with higher priority are not considered. 


\begin{tabular}{|c|c|c|c|c|c|c|c|c|c|}
\hline \multirow{3}{*}{ Analysis categories } & \multicolumn{8}{|c|}{ SM $125 \mathrm{GeV}$ Higgs boson expected signal } & \multirow{3}{*}{$\mathrm{S} /(\mathrm{S}+\mathrm{B})$} \\
\hline & \multirow{2}{*}{ Total } & \multicolumn{2}{|c|}{ Target } & \multicolumn{3}{|c|}{ Fraction of total events } & \multirow[b]{2}{*}{ Top } & \multirow{2}{*}{$\begin{array}{c}\sigma_{\text {eff }} \\
(\mathrm{GeV})\end{array}$} & \\
\hline & & STXS bin(s) & $\operatorname{ggH}$ & $\mathrm{b} \overline{\mathrm{b}} \mathrm{H}$ & $\mathrm{qqH}$ & VH lep & & & \\
\hline $0 \mathrm{~J}$ low $p_{\mathrm{T}}^{\gamma \gamma}$ Tag0 & 296.2 & $86.6 \%$ & $97.9 \%$ & $1.1 \%$ & $0.8 \%$ & $0.1 \%$ & - & 1.89 & 0.06 \\
\hline $0 \mathrm{~J}$ low $p_{\mathrm{T}}^{\gamma \gamma}$ Tag1 & 340.0 & $88.5 \%$ & $98.0 \%$ & $1.0 \%$ & $0.8 \%$ & $0.1 \%$ & - & 2.31 & 0.03 \\
\hline $0 \mathrm{~J}$ low $p_{\mathrm{T}}^{\gamma \gamma}$ Tag2 & 279.6 & $89.3 \%$ & $98.1 \%$ & $1.0 \%$ & $0.8 \%$ & $0.1 \%$ & - & 2.53 & 0.02 \\
\hline $0 \mathrm{~J}$ high $p_{\mathrm{T}}^{\gamma \gamma}$ Tag0 & 612.4 & $81.9 \%$ & $95.6 \%$ & $1.4 \%$ & $2.6 \%$ & $0.4 \%$ & - & 1.64 & 0.09 \\
\hline $0 \mathrm{~J}$ high $p_{\mathrm{T}}^{\gamma \gamma} \mathrm{Tag} 1$ & 1114.6 & $79.4 \%$ & $95.4 \%$ & $1.3 \%$ & $2.8 \%$ & $0.4 \%$ & - & 2.19 & 0.05 \\
\hline $0 \mathrm{~J}$ high $p_{\mathrm{T}}^{\gamma \gamma}$ Tag2 & 1162.6 & $78.3 \%$ & $95.3 \%$ & $1.4 \%$ & $2.7 \%$ & $0.5 \%$ & - & 2.56 & 0.02 \\
\hline 1J low $p_{\mathrm{T}}^{\gamma \gamma} \operatorname{Tag} 0$ & 132.0 & $66.2 \%$ & $88.8 \%$ & $0.8 \%$ & $9.4 \%$ & $0.8 \%$ & $0.1 \%$ & 1.53 & 0.11 \\
\hline 1J low $p_{\mathrm{T}}^{\gamma \gamma}$ Tag1 & 340.0 & $66.3 \%$ & $88.6 \%$ & $0.8 \%$ & $9.6 \%$ & $0.9 \%$ & $0.1 \%$ & 1.95 & 0.05 \\
\hline 1J low $p_{\mathrm{T}}^{\gamma \gamma}$ Tag2 & 260.6 & $66.2 \%$ & $88.3 \%$ & $0.8 \%$ & $9.7 \%$ & $1.0 \%$ & $0.1 \%$ & 2.37 & 0.02 \\
\hline $1 \mathrm{~J}$ med $p_{\mathrm{T}}^{\gamma \gamma}$ Tag0 & 184.1 & $65.2 \%$ & $81.7 \%$ & $0.5 \%$ & $16.3 \%$ & $1.4 \%$ & $0.2 \%$ & 1.65 & 0.15 \\
\hline $1 \mathrm{~J}$ med $p_{\mathrm{T}}^{\gamma \gamma}$ Tag1 & 310.2 & $66.3 \%$ & $83.6 \%$ & $0.4 \%$ & $14.3 \%$ & $1.6 \%$ & $0.1 \%$ & 1.91 & 0.08 \\
\hline $1 \mathrm{~J}$ med $p_{\mathrm{T}}^{\gamma \gamma}$ Tag2 & 291.4 & $65.0 \%$ & $83.7 \%$ & $0.5 \%$ & $13.8 \%$ & $1.8 \%$ & $0.2 \%$ & 2.13 & 0.03 \\
\hline $1 \mathrm{~J}$ high $p_{\mathrm{T}}^{\gamma \gamma} \operatorname{Tag} 0$ & 37.3 & $61.9 \%$ & $75.7 \%$ & $0.2 \%$ & $22.8 \%$ & $1.0 \%$ & $0.2 \%$ & 1.55 & 0.30 \\
\hline 1J high $p_{\mathrm{T}}^{\gamma \gamma}$ Tag1 & 31.2 & $61.7 \%$ & $75.0 \%$ & $0.3 \%$ & $23.4 \%$ & $1.1 \%$ & $0.2 \%$ & 1.73 & 0.16 \\
\hline 1J high $p_{\mathrm{T}}^{\gamma \gamma}$ Tag2 & 80.9 & $62.2 \%$ & $76.5 \%$ & $0.2 \%$ & $21.5 \%$ & $1.6 \%$ & $0.2 \%$ & 1.97 & 0.07 \\
\hline$\geq 2 \mathrm{~J}$ low $p_{\mathrm{T}}^{\gamma \gamma}$ Tag 0 & 17.7 & $52.7 \%$ & $76.7 \%$ & $0.6 \%$ & $19.0 \%$ & $1.3 \%$ & $2.4 \%$ & 1.56 & 0.06 \\
\hline$\geq 2 \mathrm{~J}$ low $p_{\mathrm{T}}^{\gamma \gamma} \operatorname{Tag} 1$ & 57.6 & $54.0 \%$ & $74.4 \%$ & $0.6 \%$ & $20.5 \%$ & $1.4 \%$ & $3.0 \%$ & 1.88 & 0.03 \\
\hline$\geq 2 \mathrm{~J}$ low $p_{\mathrm{T}}^{\gamma \gamma}$ Tag2 & 43.9 & $50.5 \%$ & $72.7 \%$ & $0.6 \%$ & $20.8 \%$ & $1.7 \%$ & $4.2 \%$ & 2.46 & 0.01 \\
\hline$\geq 2 \mathrm{~J} \operatorname{med} p_{\mathrm{T}}^{\gamma \gamma} \operatorname{Tag} 0$ & 21.2 & $64.9 \%$ & $80.6 \%$ & $0.3 \%$ & $16.3 \%$ & $1.0 \%$ & $1.8 \%$ & 1.42 & 0.17 \\
\hline$\geq 2 \mathrm{~J}$ med $p_{\mathrm{T}}^{\gamma \gamma}$ Tag1 & 70.1 & $61.4 \%$ & $77.9 \%$ & $0.3 \%$ & $18.1 \%$ & $1.1 \%$ & $2.6 \%$ & 1.82 & 0.07 \\
\hline$\geq 2 \mathrm{~J} \operatorname{med} p_{\mathrm{T}}^{\gamma \gamma}$ Tag2 & 135.4 & $57.5 \%$ & $74.8 \%$ & $0.4 \%$ & $19.7 \%$ & $1.4 \%$ & $3.8 \%$ & 2.08 & 0.03 \\
\hline$\geq 2 \mathrm{~J}$ high $p_{\mathrm{T}}^{\gamma \gamma}$ Tag0 & 29.0 & $65.5 \%$ & $77.8 \%$ & $0.2 \%$ & $18.7 \%$ & $1.3 \%$ & $2.1 \%$ & 1.48 & 0.23 \\
\hline$\geq 2 \mathrm{~J}$ high $p_{\mathrm{T}}^{\gamma \gamma}$ Tag 1 & 52.5 & $62.3 \%$ & $76.1 \%$ & $0.2 \%$ & $19.6 \%$ & $1.5 \%$ & $2.6 \%$ & 1.76 & 0.11 \\
\hline$\geq 2 \mathrm{~J}$ high $p_{\mathrm{T}}^{\gamma \gamma}$ Tag2 & 45.5 & $58.4 \%$ & $73.8 \%$ & $0.2 \%$ & $20.4 \%$ & $1.9 \%$ & $3.7 \%$ & 1.92 & 0.05 \\
\hline BSM $200<p_{\mathrm{T}}^{\gamma \gamma}<300$ Tag0 & 30.7 & $75.8 \%$ & $77.5 \%$ & $0.2 \%$ & $19.4 \%$ & $1.2 \%$ & $1.6 \%$ & 1.41 & 0.39 \\
\hline BSM $200<p_{\mathrm{T}}^{\gamma \gamma}<300$ Tag1 & 39.6 & $69.9 \%$ & $73.8 \%$ & $0.1 \%$ & $21.5 \%$ & $1.7 \%$ & $2.8 \%$ & 1.90 & 0.11 \\
\hline BSM $300<p_{\mathrm{T}}^{\gamma \gamma}<450$ Tag0 & 15.5 & $74.8 \%$ & $76.3 \%$ & $0.1 \%$ & $19.7 \%$ & $1.7 \%$ & $2.2 \%$ & 1.53 & 0.34 \\
\hline BSM $300<p_{\mathrm{T}}^{\gamma \gamma}<450$ Tag1 & 2.6 & $66.3 \%$ & $67.9 \%$ & $0.1 \%$ & $22.5 \%$ & $2.6 \%$ & $7.0 \%$ & 1.42 & 0.09 \\
\hline $\mathrm{BSM} 450<p_{\mathrm{T}}^{\gamma \gamma}<650$ & 3.1 & $58.1 \%$ & $61.8 \%$ & $0.1 \%$ & $30.0 \%$ & $2.4 \%$ & $5.6 \%$ & 1.55 & 0.20 \\
\hline $\mathrm{BSM} p_{\mathrm{T}}^{\gamma \gamma}>650$ & 0.9 & $72.5 \%$ & $72.3 \%$ & $0.1 \%$ & $21.0 \%$ & $2.9 \%$ & $3.8 \%$ & 1.21 & 0.36 \\
\hline
\end{tabular}

Table 3. The expected number of signal events for $m_{\mathrm{H}}=125 \mathrm{GeV}$ in analysis categories targeting ggH production, excluding those targeting the VBF-like phase space, shown for an integrated luminosity of $137 \mathrm{fb}^{-1}$. The fraction of the total number of events arising from each production mode in each analysis category is provided, as is the fraction of events originating from the targeted STXS bin or bins. Entries with values less than $0.05 \%$ are not shown. Here qqH includes contributions

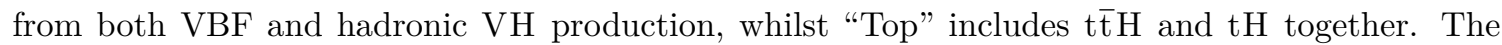
$\sigma_{\text {eff }}$, defined as the smallest interval containing $68.3 \%$ of the $m_{\gamma \gamma}$ distribution, is listed for each analysis category. The final column shows the expected ratio of signal to signal-plus-background, $\mathrm{S} /(\mathrm{S}+\mathrm{B})$, where $\mathrm{S}$ and $\mathrm{B}$ are the numbers of expected signal and background events in a $\pm 1 \sigma_{\text {eff }}$ window centred on $m_{\mathrm{H}}$. 


\begin{tabular}{|c|c|c|c|c|c|}
\hline \multirow{2}{*}{ STXS bin } & \multirow{2}{*}{$\begin{array}{c}\text { Definition } \\
\text { units of } p_{\mathrm{T}}^{\mathrm{H}}, m_{\mathrm{jj}} \text { and } p_{\mathrm{T}}^{\mathrm{Hjj}} \text { in } \mathrm{GeV}\end{array}$} & \multicolumn{3}{|c|}{ Fraction of cross section } & \multirow{2}{*}{$\sigma_{\mathrm{SM}} \mathcal{B}(\mathrm{fb})$} \\
\hline & & VBF & $\mathrm{q} \overline{\mathrm{q}}^{\prime} \rightarrow \mathrm{W}\left(\mathrm{q} \overline{\mathrm{q}}^{\prime}\right) \mathrm{H}$ & $\mathrm{q} \overline{\mathrm{q}} \rightarrow \mathrm{Z}(\mathrm{q} \overline{\mathrm{q}}) \mathrm{H}$ & \\
\hline qqH forward & $\left|y_{\mathrm{H}}\right|>2.5$ & $6.69 \%$ & $12.57 \%$ & $9.84 \%$ & 0.98 \\
\hline $\mathrm{qqH} 0 \mathrm{~J}$ & Exactly 0 jets & $6.95 \%$ & $5.70 \%$ & $3.73 \%$ & 0.77 \\
\hline qqH $1 \mathrm{~J}$ & Exactly 1 jet & $32.83 \%$ & $31.13 \%$ & $25.03 \%$ & 3.82 \\
\hline $\mathrm{qqH} m_{\mathrm{jj}}<60$ & At least 2 jets, $m_{\mathrm{jj}}<60$ & $1.36 \%$ & $3.58 \%$ & $2.72 \%$ & 0.23 \\
\hline qqH VH-like & At least 2 jets, $60<m_{\mathrm{jj}}<120$ & $2.40 \%$ & $29.43 \%$ & $28.94 \%$ & 1.23 \\
\hline $\mathrm{qqH} 120<m_{\mathrm{jj}}<350$ & At least 2 jets, $120<m_{\mathrm{jj}}<350$ & $12.34 \%$ & $13.92 \%$ & $12.59 \%$ & 1.53 \\
\hline $\mathrm{qqH}$ VBF-like low $m_{\mathrm{jj}}$ low $p_{\mathrm{T}}^{\mathrm{Hjj}}$ & $\begin{array}{c}\text { At least } 2 \text { jets, } p_{\mathrm{T}}^{\mathrm{H}}<200 \\
350<m_{\mathrm{jj}}<700, p_{\mathrm{T}}^{\mathrm{Hjj}}<25\end{array}$ & $10.26 \%$ & $0.44 \%$ & $0.35 \%$ & 0.90 \\
\hline qqH VBF-like low $m_{\mathrm{jj}}$ high $p_{\mathrm{T}}^{\mathrm{Hjj}}$ & $\begin{array}{l}\text { At least } 2 \text { jets, } p_{\mathrm{T}}^{\mathrm{H}}<200, \\
350<m_{\mathrm{jj}}<700, p_{\mathrm{T}}^{\mathrm{H} j \mathrm{j}}>25\end{array}$ & $3.85 \%$ & $1.86 \%$ & $1.74 \%$ & 0.39 \\
\hline qqH VBF-like high $m_{\mathrm{jj}}$ low $p_{\mathrm{T}}^{\mathrm{Hjj}}$ & $\begin{array}{l}\text { At least } 2 \text { jets, } p_{\mathrm{T}}^{\mathrm{H}}<200 \\
m_{\mathrm{jj}}>700, p_{\mathrm{T}}^{\mathrm{Hjj}}<25\end{array}$ & $15.09 \%$ & $0.09 \%$ & $0.08 \%$ & 1.30 \\
\hline qqH VBF-like high $m_{\mathrm{jj}}$ high $p_{\mathrm{T}}^{\mathrm{Hjj}}$ & $\begin{array}{c}\text { At least } 2 \text { jets, } p_{\mathrm{T}}^{\mathrm{H}}<200 \\
m_{\mathrm{jj}}>700, p_{\mathrm{T}}^{\mathrm{Hjj}}>25\end{array}$ & $4.25 \%$ & $0.40 \%$ & $0.39 \%$ & 0.38 \\
\hline $\mathrm{qqH}$ BSM & At least 2 jets, $m_{\mathrm{jj}}>350, p_{\mathrm{T}}^{\mathrm{H}}>200$ & $3.98 \%$ & $0.88 \%$ & $0.71 \%$ & 0.37 \\
\hline
\end{tabular}

Table 4. Definition of the qqH STXS bins. The product of the cross section and branching fraction $(\mathcal{B})$, evaluated at $\sqrt{s}=13 \mathrm{TeV}$ and $m_{\mathrm{H}}=125 \mathrm{GeV}$, is given for each bin in the last column. The fraction of the total production mode cross section from each STXS bin is also shown. Unless stated otherwise, the STXS bins are defined for $\left|y_{\mathrm{H}}\right|<2.5$. Events with $\left|y_{\mathrm{H}}\right|>2.5$ are mostly outside of the experimental acceptance and therefore have a negligible contribution to all analysis categories.

\subsection{Event categories for VBF production}

In the STXS framework, the qqH production mode includes both VBF events and VH events where the vector boson decays hadronically. Within qqH production, there are five STXS bins that correspond to typical VBF-like events, with a single bin for VH-like events. The precise definitions of the qqH STXS bins are given in table 4. These correspond to the orange entries in figure 1.

Events with a dijet system characteristic of the VBF production mode have a dedicated categorisation scheme in this analysis, described in this section. Those events where the dijet is instead consistent with the decay of a vector boson are categorised separately, as described in section 6.3. No analysis categories are constructed to target the zero or one jet qqH STXS bins, nor those with $m_{\mathrm{jj}}<60 \mathrm{GeV}$ or $120<m_{\mathrm{jj}}<350 \mathrm{GeV}$.

Following the STXS binning scheme, the particle-level definition of the VBF-like dijet system requires two jets with $p_{\mathrm{T}}>30 \mathrm{GeV}$, and whose $m_{\mathrm{jj}}>350 \mathrm{GeV}$. These bins are defined analogously for $\mathrm{EW} \mathrm{qqH}$ production as well as from $\mathrm{ggH}$ production. When constructing the corresponding analysis categories at reconstruction level, a dijet preselection is applied that requires two jets within $|\eta|<4.7$, with $p_{\mathrm{T}}>40(30) \mathrm{GeV}$ for the leading (subleading) jet, in addition to $m_{\mathrm{jj}}>350 \mathrm{GeV}$. Jets are also required to pass a threshold on a pileup identification score.

The so-called dijet BDT is trained to estimate the probability that an event passing the VBF preselection originated from VBF, $\mathrm{ggH}$, or non-Higgs boson SM diphoton production. The inputs to the dijet BDT include various jet kinematic and angular variables, as well as the $p_{\mathrm{T}} / m_{\gamma \gamma}$ of each photon and angular variables involving both jets and photons. These 
inputs for $\mathrm{VBF}, \mathrm{ggH}$, and non-Higgs boson SM production of two prompt photons are taken from simulation. However, the modelling of backgrounds, where at least one of the two photons is a misreconstructed jet, is poor, predominantly due to the fact that very few simulated events pass the selection criteria. In this analysis, an approach is adopted whereby the simulated background events with nonprompt photons are replaced with data from a dedicated control sample.

The control sample is defined using the sideband of the photon ID BDT distribution, by requiring at least one photon ID BDT score to be below -0.5 . The events in this control sample can potentially have both a different normalisation and different kinematic properties from those in the signal region. To correct this, the events are reweighted in bins of the $p_{\mathrm{T}}$ and $|\eta|$ of the photon passing the ID BDT requirement. The required weights are derived from simulation, by estimating both the fraction of background events that contain nonprompt photons and the ratio of the expected number of events in the signal region to the control sample. The product of these two factors is applied as a weight to each data event in the control sample, and these reweighted events are subsequently used to train the dijet BDT.

The resulting distributions of the dijet BDT input variables are compared to the $m_{\gamma \gamma}$ sideband data and are found to be in reasonable agreement. Furthermore, the increase in the number of events available for the training of the dijet BDT leads to an improvement in its discrimination power.

The two independent output probabilities of the dijet BDT, taken to be the VBF probability and the $\mathrm{ggH}$ probability, are validated in $\mathrm{Z} \rightarrow$ ee + jets events with the electrons reconstructed as photons. The dijet preselection criteria required to enter the VBF-like analysis categories are also applied to the $\mathrm{Z} \rightarrow$ ee + jets events. The VBF probability distribution in simulation and data sidebands is shown in the left plot of figure 7 , while the right plot demonstrates good agreement between data and simulation in $\mathrm{Z} \rightarrow$ ee + jets events. A similar level of agreement is observed in the $\mathrm{ggH}$ probability distribution.

Due to the use of the data control sample with photon ID BDT score below -0.5 in the dijet BDT training, an additional requirement that the two photons have a photon ID BDT score of larger than -0.2 is placed on events entering the VBF-like analysis categories. The final analysis categories are constructed following the structure of the STXS binning scheme. Events can be assigned to analysis categories targeting one of five VBF-like STXS bins, as shown in figure 1. The first is defined as having a high $p_{\mathrm{T}}^{\mathrm{H}}$, with a threshold set at $200 \mathrm{GeV}$. The remaining four bins have $p_{\mathrm{T}}^{\mathrm{H}}<200 \mathrm{GeV}$. They are defined by boundaries on $p_{\mathrm{T}}^{\mathrm{H}}$ and $p_{\mathrm{T}}^{\mathrm{Hjj}}$ at $25 \mathrm{GeV}$ and $m_{\mathrm{jj}}$ at $700 \mathrm{GeV}$. The $p_{\mathrm{T}}^{\mathrm{Hjj}}$ threshold is chosen to separate events containing two jets from those containing three or more, which are referred to as two-jet-like $\left(p_{\mathrm{T}}^{\mathrm{Hjj}}<25 \mathrm{GeV}\right)$ and three-jet-like $\left(p_{\mathrm{T}}^{\mathrm{Hjj}}>25 \mathrm{GeV}\right)$ bins, respectively. The analysis categories are defined using the reconstructed observables corresponding to each particle-level quantity; these are the $p_{\mathrm{T}}^{\gamma \gamma}$, the reconstructed $m_{\mathrm{jj}}$, and the reconstructed $p_{\mathrm{T}}^{\mathrm{Hjj}}$.

Events are further divided into analysis categories using both the dijet BDT output probabilities and the diphoton BDT score. For each of the five STXS bins, a set of analysis categories is constructed with events originating from VBF production considered as signal. An optimisation is performed defining lower bounds on the dijet VBF probability and 

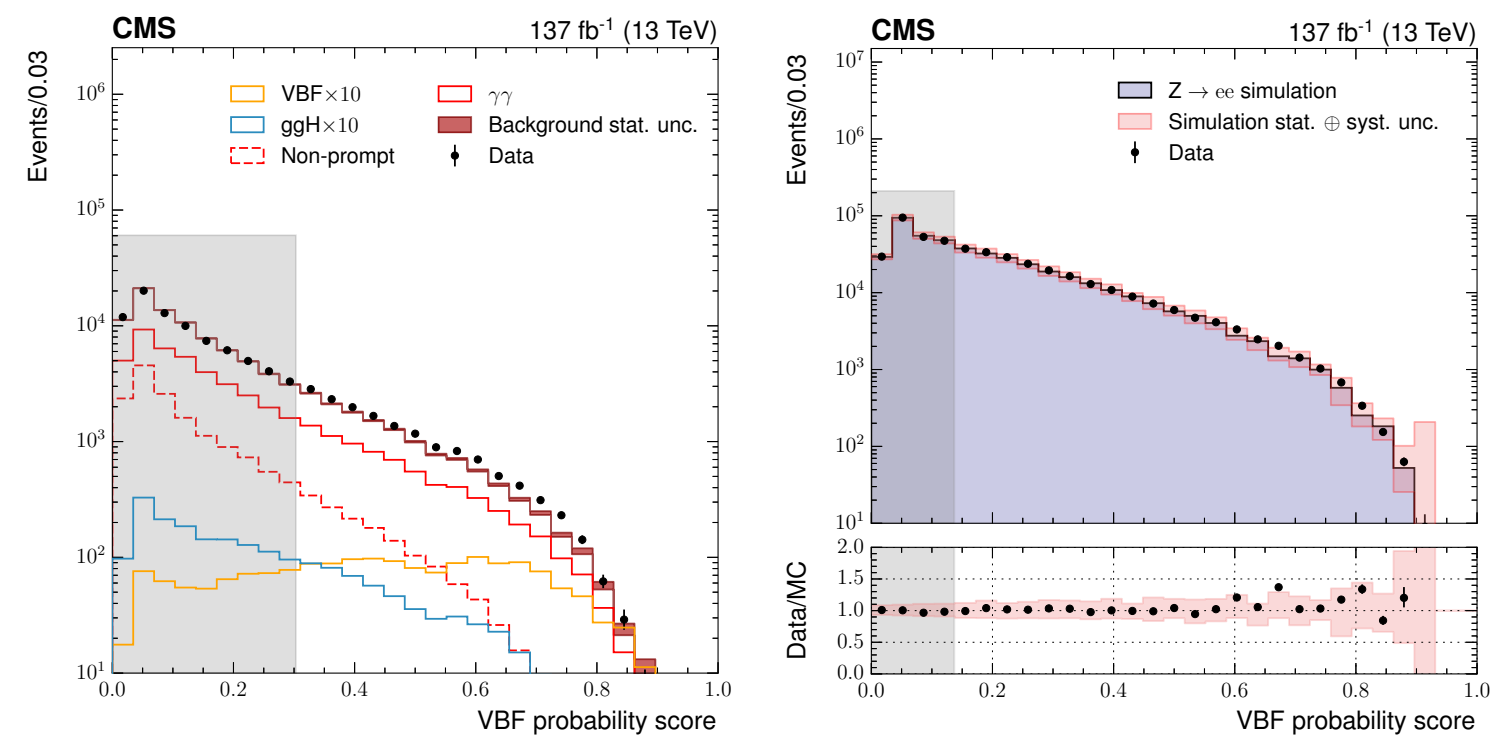

Figure 7. The left plot shows the distribution of the dijet BDT output VBF probability in events with $m_{\gamma \gamma}$ in the range 100-120 or 130-180 GeV, for data events passing the dijet preselection (black points), and for simulated background events (red band). Histograms are also shown for different components of the simulated background in red. The orange histogram corresponds to simulated VBF signal events, with the ggH events shown in blue (both $\times 100)$. The right plot shows the same distribution in $\mathrm{Z} \rightarrow$ ee events where the electrons are reconstructed as photons. The points show the score for data, the histogram shows the score for simulated Drell-Yan events, including statistical and systematic uncertainties (pink band). The regions shaded grey contain VBF probability values below the lowest threshold used to define an analysis category. The full data set collected in 20162018 and the corresponding simulation are shown.

diphoton BDT score, with an upper bound on the dijet $\operatorname{ggH}$ probability. Two analysis categories are constructed to target each STXS bin, the expected composition of which is given in table 5 .

An additional set of analysis categories is defined covering the four STXS bins with $p_{\mathrm{T}}^{\mathrm{H}}<200 \mathrm{GeV}$, but considering ggH events as signal instead of VBF. Two analysis categories targeting the set of four STXS bins together are constructed. Here lower bounds are set on the dijet $\mathrm{ggH}$ probability and diphoton BDT score, with an upper bound placed on the dijet VBF probability. The expected composition of these is also given in table 5 .

\subsection{Event categories for hadronic VH production}

In the EW qqH STXS binning scheme, there is a bin representing hadronic VH production, defined at the particle level by $60<m_{\mathrm{jj}}<120 \mathrm{GeV}$. Analysis categories targeting this bin are constructed in a similar way to those targeting VBF-like dijet events. The principal difference is in the selection of the two jets. The hadronic VH preselection requires two jets within $|\eta|<2.4$ and with $p_{\mathrm{T}}>30 \mathrm{GeV}$, and satisfying a pileup jet identification criterion. In addition, the reconstructed $m_{\mathrm{jj}}$ is required to be consistent with a decay of a vector boson, $60<m_{\mathrm{jj}}<120 \mathrm{GeV}$. 


\begin{tabular}{|c|c|c|c|c|c|c|c|c|c|}
\hline \multirow{3}{*}{ Analysis categories } & \multicolumn{8}{|c|}{ SM $125 \mathrm{GeV}$ Higgs boson expected signal } & \multirow{3}{*}{$\mathrm{S} /(\mathrm{S}+\mathrm{B})$} \\
\hline & \multirow{2}{*}{ Total } & \multicolumn{2}{|c|}{ Target } & \multicolumn{3}{|c|}{ Fraction of total events } & \multirow[b]{2}{*}{ Top } & \multirow{2}{*}{$\begin{array}{c}\sigma_{\text {eff }} \\
(\mathrm{GeV})\end{array}$} & \\
\hline & & STXS bin(s) & $\operatorname{ggH}$ & VBF & VH had & VH lep & & & \\
\hline ggH VBF-like Tag0 & 14.1 & $37.7 \%$ & $65.9 \%$ & $27.3 \%$ & $3.8 \%$ & $0.8 \%$ & $2.3 \%$ & 1.85 & 0.14 \\
\hline ggH VBF-like Tag1 & 32.5 & $30.2 \%$ & $61.3 \%$ & $29.8 \%$ & $4.1 \%$ & $1.1 \%$ & $3.7 \%$ & 1.83 & 0.10 \\
\hline $\mathrm{qqH}$ low $m_{\mathrm{jj}}$ low $p_{\mathrm{T}}^{\mathrm{H} \mathrm{jj}} \operatorname{Tag} 0$ & 17.2 & $48.2 \%$ & $36.6 \%$ & $62.6 \%$ & $0.4 \%$ & $0.1 \%$ & $0.3 \%$ & 1.89 & 0.20 \\
\hline $\mathrm{qqH}$ low $m_{\mathrm{jj}}$ low $p_{\mathrm{T}}^{\mathrm{H} j \mathrm{j}} \mathrm{Tag} 1$ & 13.5 & $48.5 \%$ & $35.5 \%$ & $63.4 \%$ & $0.6 \%$ & $0.1 \%$ & $0.3 \%$ & 1.74 & 0.19 \\
\hline $\mathrm{qqH}$ high $m_{\mathrm{jj}}$ low $p_{\mathrm{T}}^{\mathrm{H} j \mathrm{j}}$ Tag0 & 27.0 & $70.4 \%$ & $17.1 \%$ & $82.7 \%$ & $0.2 \%$ & - & $0.1 \%$ & 1.78 & 0.49 \\
\hline $\mathrm{qqH}$ high $m_{\mathrm{jj}}$ low $p_{\mathrm{T}}^{\mathrm{Hjj}}$ Tag1 & 12.9 & $58.2 \%$ & $20.8 \%$ & $78.7 \%$ & $0.3 \%$ & $0.1 \%$ & $0.2 \%$ & 1.99 & 0.27 \\
\hline $\mathrm{qqH}$ low $m_{\mathrm{jj}}$ high $p_{\mathrm{T}}^{\mathrm{Hjj}}$ Tag0 & 10.4 & $15.0 \%$ & $56.0 \%$ & $41.3 \%$ & $1.3 \%$ & $0.4 \%$ & $1.0 \%$ & 1.92 & 0.12 \\
\hline $\mathrm{qqH}$ low $m_{\mathrm{jj}}$ high $p_{\mathrm{T}}^{\mathrm{Hjj}} \operatorname{Tag} 1$ & 20.2 & $17.0 \%$ & $57.9 \%$ & $36.9 \%$ & $2.4 \%$ & $0.7 \%$ & $2.1 \%$ & 1.74 & 0.08 \\
\hline $\mathrm{qqH}$ high $m_{\mathrm{jj}}$ high $p_{\mathrm{T}}^{\mathrm{Hjj}}$ Tag0 & 18.1 & $25.6 \%$ & $28.1 \%$ & $70.8 \%$ & $0.4 \%$ & $0.1 \%$ & $0.5 \%$ & 1.88 & 0.29 \\
\hline $\mathrm{qqH}$ high $m_{\mathrm{jj}}$ high $p_{\mathrm{T}}^{\mathrm{H} j \mathrm{j}}$ Tag1 & 17.5 & $23.8 \%$ & $39.5 \%$ & $57.8 \%$ & $0.9 \%$ & $0.3 \%$ & $1.5 \%$ & 1.98 & 0.13 \\
\hline qqH BSM Tag0 & 11.2 & $71.2 \%$ & $24.4 \%$ & $74.8 \%$ & $0.1 \%$ & $0.1 \%$ & $0.6 \%$ & 1.62 & 0.56 \\
\hline qqH BSM Tag1 & 6.8 & $56.4 \%$ & $36.9 \%$ & $59.9 \%$ & $1.1 \%$ & $0.4 \%$ & $1.7 \%$ & 1.67 & 0.39 \\
\hline qqH VH-like Tag0 & 16.3 & $55.8 \%$ & $36.5 \%$ & $2.8 \%$ & $55.0 \%$ & $1.4 \%$ & $4.2 \%$ & 1.72 & 0.25 \\
\hline qqH VH-like Tag1 & 47.1 & $26.8 \%$ & $64.9 \%$ & $4.7 \%$ & $26.4 \%$ & $1.2 \%$ & $2.9 \%$ & 1.66 & 0.13 \\
\hline
\end{tabular}

Table 5. The expected number of signal events for $m_{\mathrm{H}}=125 \mathrm{GeV}$ in analysis categories targeting VBF-like phase space and VH production in which the vector boson decays hadronically, shown for an integrated luminosity of $137 \mathrm{fb}^{-1}$. The fraction of the total number of events arising from each production mode in each analysis category is provided, as is the fraction of events originating from the targeted STXS bin or bins. Entries with values less than $0.05 \%$ are not shown. Here ggH includes contributions from the ggZ $(\mathrm{q} \overline{\mathrm{q}}) \mathrm{H}$ and $\mathrm{b} \overline{\mathrm{b}} \mathrm{H}$ production modes, whilst "Top" represents both $\mathrm{t} \overline{\mathrm{t}} \mathrm{H}$ and $\mathrm{t} \mathrm{H}$ production together. The $\sigma_{\text {eff }}$, defined as the smallest interval containing $68.3 \%$ of the $m_{\gamma \gamma}$ distribution, is listed for each analysis category. The final column shows the expected ratio of signal to signal-plus-background, $\mathrm{S} /(\mathrm{S}+\mathrm{B})$, where $\mathrm{S}$ and $\mathrm{B}$ are the numbers of expected signal and background events in a $\pm 1 \sigma_{\text {eff }}$ window centred on $m_{\mathrm{H}}$.

A BDT referred to as the $\mathrm{VH}$ hadronic BDT is trained with $\mathrm{VH}$ hadronic events as signal, against ggH and non-Higgs boson SM diphoton production together as background. The training events of $\mathrm{VH}, \mathrm{ggH}$, and $\mathrm{SM}$ production of two prompt photons are taken from simulation. The remaining background containing nonprompt photons is derived from a control sample in the same way as that employed for the dijet BDT training. The control sample is defined by requiring that at least one photon has a photon ID BDT score of less than -0.5 , but otherwise passes the $\mathrm{VH}$ hadronic preselection. The resulting events are weighted to reproduce the expected number of background events and used in the BDT training of the $\mathrm{VH}$ hadronic BDT.

The input variables for the VH hadronic BDT are similar to those for the dijet BDT. Variables that aid in identifying events consistent with the vector boson decay are added, including the cosine of the difference of two angles: that of the diphoton system in the diphoton-dijet centre-of-mass frame, and that of the diphoton-dijet system in the lab frame.

The final two analysis categories use the output scores of both the VH hadronic BDT and the diphoton BDT to increase sensitivity, in addition to requiring a photon ID BDT score of greater than -0.2 . 

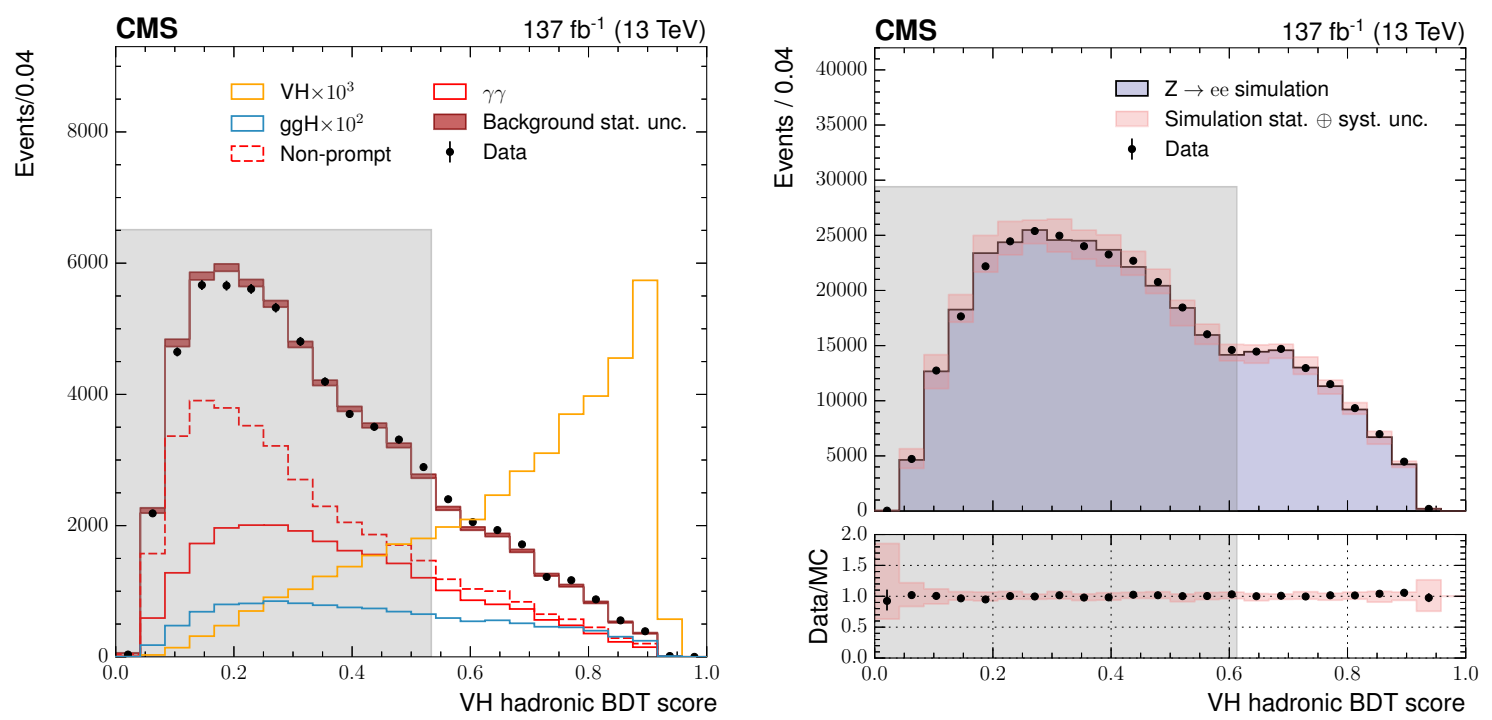

Figure 8. The left plot shows the distribution of the VH hadronic BDT output score in events with $m_{\gamma \gamma}$ in the range $100-120$ or $130-180 \mathrm{GeV}$, for data events passing the preselection (black points), and for simulated background events (red band). Histograms are also shown for different components of the simulated background in red. The sum of all background distributions is scaled to the data. The orange histogram corresponds to simulated VH hadronic signal events. The right plot shows the same distribution in $\mathrm{Z} \rightarrow$ ee + jets events where the electrons are reconstructed as photons. The points show the score for data, the histogram shows the score for simulated Drell-Yan events, including statistical and systematic uncertainties (pink band). The regions shaded grey contain VH hadronic BDT scores below the lowest threshold used to define an analysis category. The full data set collected in 2016-2018 and the corresponding simulation are shown.

The output score of the VH hadronic BDT in simulation and data sidebands is shown in the left plot of figure 8. The VH hadronic BDT is also validated in $\mathrm{Z} \rightarrow$ ee + jets events with electrons reconstructed as photons, after the VH hadronic preselection is applied. The two distributions in simulation and data are shown in the right plot figure 8 and exhibit good agreement.

The expected signal and background yields in each VBF and hadronic VH analysis category are shown in table 5.

\subsection{Event categories for leptonic VH production}

The analysis categories described here target events in which the Higgs boson is produced in association with a $\mathrm{W}$ or $\mathrm{Z}$ vector boson that subsequently decays leptonically. Depending on the particular leptonic decay mode of the vector boson, the possible final states include zero, one, or two charged leptons. The full definitions of each VH leptonic STXS bin are given in table 6 , corresponding to the green entries in figure 1 . The bins are defined using $p_{\mathrm{T}}^{\mathrm{V}}$ and the number of jets in the event.

For each of the three channels, a dedicated BDT classifier is used to discriminate between the VH signal and background events. Each of these three BDTs are trained on simulated signal and background events. The exception is the zero-lepton final state, for 


\begin{tabular}{|c|c|c|c|c|c|}
\hline \multirow{2}{*}{ STXS bin } & \multirow{2}{*}{$\begin{array}{c}\text { Definition } \\
\text { units of } p_{\mathrm{T}}^{\mathrm{V}} \text { in } \mathrm{GeV}\end{array}$} & \multicolumn{3}{|c|}{ Fraction of cross section } & \multirow{2}{*}{$\sigma_{\mathrm{SM}} \mathcal{B}(\mathrm{fb})$} \\
\hline & & $\mathrm{q} \overline{\mathrm{q}}^{\prime} \rightarrow \mathrm{WH}$ & $\mathrm{q} \overline{\mathrm{q}} \rightarrow \mathrm{ZH}$ & $\mathrm{gg} \rightarrow \mathrm{ZH}$ & \\
\hline WH lep forward & & $12.13 \%$ & - & - & 0.123 \\
\hline ZH lep forward & $\left|y_{\mathrm{H}}\right|>2.5$ & - & $11.21 \%$ & - & 0.058 \\
\hline ggZH lep forward & & - & - & $2.71 \%$ & 0.002 \\
\hline WH lep $p_{\mathrm{T}}^{\mathrm{V}}<75$ & No jet requirements, $p_{\mathrm{T}}^{\mathrm{V}}<75$ & $46.55 \%$ & - & - & 0.473 \\
\hline WH lep $75<p_{\mathrm{T}}^{\mathrm{V}}<150$ & No jet requirements, $75<p_{\mathrm{T}}^{\mathrm{V}}<150$ & $29.30 \%$ & - & - & 0.298 \\
\hline WH lep 0J $150<p_{\mathrm{T}}^{\mathrm{V}}<250$ & Exactly 0 jets, $150<p_{\mathrm{T}}^{\mathrm{V}}<250$ & $5.10 \%$ & - & - & 0.052 \\
\hline WH lep $\geq 1 \mathrm{~J} 150<p_{\mathrm{T}}^{\mathrm{V}}<250$ & At least 1 jet, $150<p_{\mathrm{T}}^{\mathrm{V}}<250$ & $3.97 \%$ & - & - & 0.040 \\
\hline WH lep $p_{\mathrm{T}}^{\mathrm{V}}>250$ & No jet requirements, $p_{\mathrm{T}}^{\mathrm{V}}>250$ & $2.95 \%$ & - & - & 0.030 \\
\hline ZH lep $p_{\mathrm{T}}^{\mathrm{V}}<75$ & No jet requirements, $p_{\mathrm{T}}^{\mathrm{V}}<75$ & - & $45.65 \%$ & - & 0.237 \\
\hline ZH lep $75<p_{\mathrm{T}}^{\mathrm{V}}<150$ & No jet requirements, $75<p_{\mathrm{T}}^{\mathrm{V}}<150$ & - & $30.70 \%$ & - & 0.160 \\
\hline ZH lep 0J $150<p_{\mathrm{T}}^{\mathrm{V}}<250$ & Exactly 0 jets, $150<p_{\mathrm{T}}^{\mathrm{V}}<250$ & - & $5.16 \%$ & - & 0.027 \\
\hline ZH lep $\geq 1 \mathrm{~J} 150<p_{\mathrm{T}}^{\mathrm{V}}<250$ & At least 1 jet, $150<p_{\mathrm{T}}^{\mathrm{V}}<250$ & - & $4.27 \%$ & - & 0.022 \\
\hline ZH lep $p_{\mathrm{T}}^{\mathrm{V}}>250$ & No jet requirements, $p_{\mathrm{T}}^{\mathrm{V}}>250$ & - & $3.01 \%$ & - & 0.016 \\
\hline ggZH lep $p_{\mathrm{T}}^{\mathrm{V}}<75$ & No jet requirements, $p_{\mathrm{T}}^{\mathrm{V}}<75$ & - & - & $15.96 \%$ & 0.013 \\
\hline ggZH lep $75<p_{\mathrm{T}}^{\mathrm{V}}<150$ & No jet requirements, $75<p_{\mathrm{T}}^{\mathrm{V}}<150$ & - & - & $43.32 \%$ & 0.036 \\
\hline ggZH lep 0J $150<p_{\mathrm{T}}^{\mathrm{V}}<250$ & Exactly 0 jets, $150<p_{\mathrm{T}}^{\mathrm{V}}<250$ & - & - & $9.08 \%$ & 0.008 \\
\hline ggZH lep $\geq 1 \mathrm{~J} 150<p_{\mathrm{T}}^{\mathrm{V}}<250$ & At least 1 jet, $150<p_{\mathrm{T}}^{\mathrm{V}}<250$ & - & - & $20.49 \%$ & 0.017 \\
\hline $\operatorname{ggZH}$ lep $p_{\mathrm{T}}^{\mathrm{V}}>250$ & No jet requirements, $p_{\mathrm{T}}^{\mathrm{V}}>250$ & - & - & $8.45 \%$ & 0.007 \\
\hline
\end{tabular}

Table 6. Definition of the VH leptonic STXS bins. The product of the cross section and branching fraction $(\mathcal{B})$, evaluated at $\sqrt{s}=13 \mathrm{TeV}$ and $m_{\mathrm{H}}=125 \mathrm{GeV}$, is given for each bin in the last column. The fraction of the total production mode cross section from each STXS bin is also shown. Unless stated otherwise, the STXS bins are defined for $\left|y_{\mathrm{H}}\right|<2.5$. Events with $\left|y_{\mathrm{H}}\right|>2.5$ are mostly outside of the experimental acceptance and therefore have a negligible contribution to all analysis categories. Only leptonic decays of the $\mathrm{W}$ and $\mathrm{Z}$ bosons are included in these definitions.

which some simulated backgrounds are replaced by events derived from data, as described below. The simulated SM background processes include photons plus jets, Drell-Yan, diboson production, and top quark pair production. The production modes of the Higgs boson other than VH are also treated as backgrounds. Where there are a sufficient number of expected signal events, the categorisation regions are further split into analysis categories sensitive to merged groups of STXS bins.

The categorisation region with two same-flavour reconstructed leptons in the final state focuses on the $\mathrm{Z}(\ell \ell) \mathrm{H}$ production mode. Additional selection criteria are imposed to select two leptons consistent with the decay of a $\mathrm{Z}$ boson, including a requirement that the dilepton mass $\left(m_{\ell \ell}\right)$ is between 60 and $120 \mathrm{GeV}$.

The so-called ZH leptonic BDT is used to discriminate the $\mathrm{Z}(\ell \ell) \mathrm{H}$ signal events from backgrounds including both other Higgs boson production modes and non-Higgs-boson SM processes. Its input variables are kinematic properties of the photons, leptons, and jets present in the event, including angular variables describing the separation between the photons and leptons. In addition, jet identification variables such as the $\mathrm{b}$ tag score are used as inputs, which helps to discriminate against backgrounds containing top quarks.

The distributions of the ZH leptonic BDT score for simulated signal and background events, along with the same for the data sidebands, are shown in figure 9 . With the 

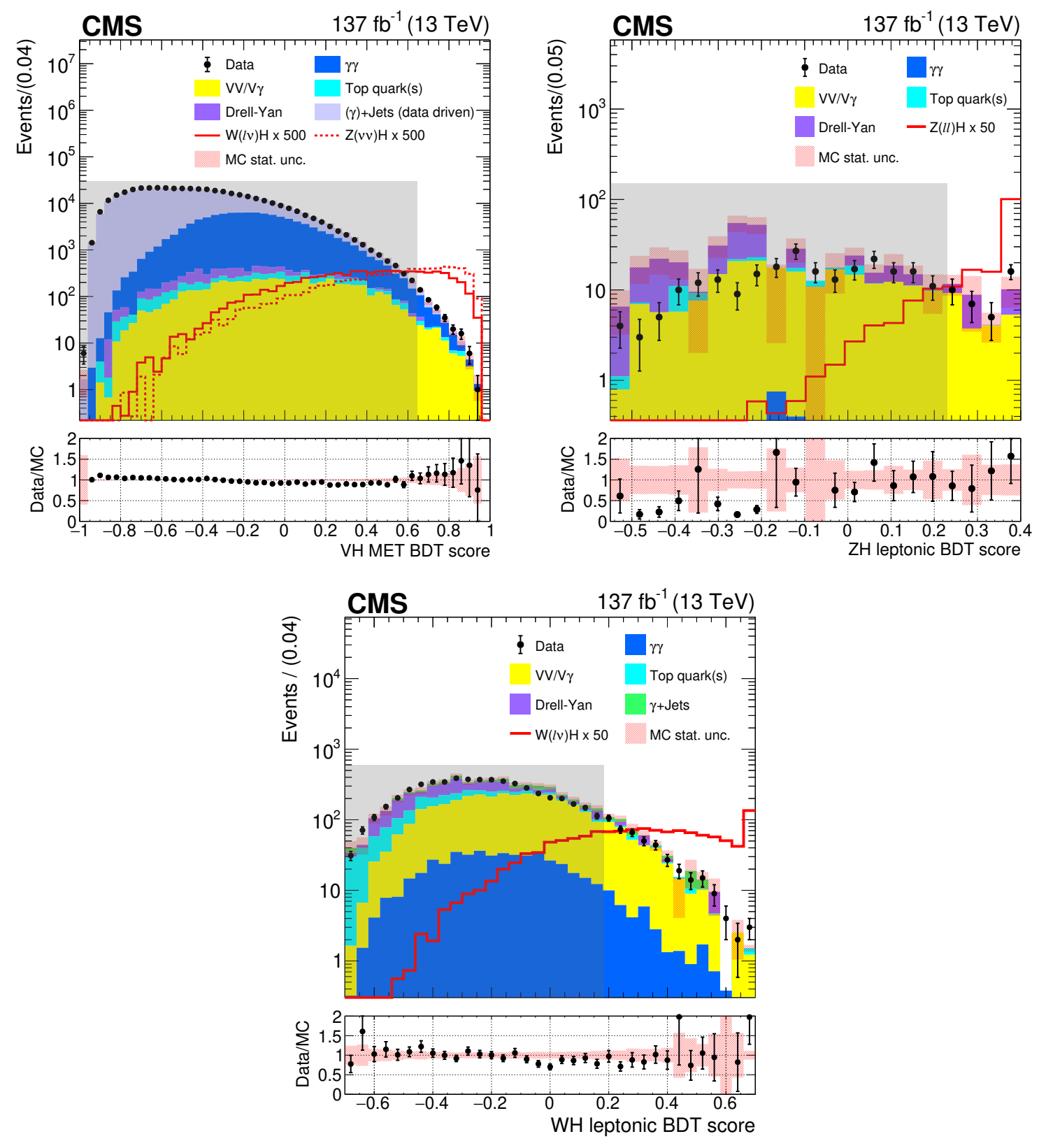

Figure 9. Output scores for the three VH leptonic BDTs. The VH MET BDT is shown in the upper left, with the $\mathrm{ZH}$ leptonic BDT in the upper right, and the WH leptonic BDT below. In each case, the signal and background simulation are shown as histograms with the data as black points. Events are taken from the $m_{\gamma \gamma}$ sidebands, satisfying either $100<m_{\gamma \gamma}<120 \mathrm{GeV}$ or $130<m_{\gamma \gamma}<180 \mathrm{GeV}$. The statistical uncertainty in the data points is denoted as vertical bars and that on the background simulation by the pink band. The simulated signal and background distributions are normalised to the luminosity of the data. To increase its visibility, the signal is scaled by a factor of 500 for the VH MET BDT, with a factor of 50 applied for both ZH leptonic and WH leptonic BDTs. The regions shaded grey are not considered in the analysis. The full data set collected in 2016-2018 and the corresponding simulation are shown. 
available data set, this categorisation region is not sensitive to the corresponding individual STXS bins. For this reason, further splitting of the analysis categories is not performed. The sensitivity to inclusive leptonic $\mathrm{ZH}$ production is maximised by defining two analysis categories using the BDT score.

To gain sensitivity to the $\mathrm{W}(\ell \nu) \mathrm{H}$ production mode, events with one reconstructed lepton are selected. Additional selection criteria are applied on the photon ID BDT to further reject background events containing nonprompt photons, and on the invariant mass of the reconstructed lepton with each photon to reduce the contamination of Drell-Yan events with an electron misidentified as a photon.

With this selection, the WH leptonic BDT is trained with simulated $\mathrm{W}(\ell \nu) \mathrm{H}$ signal events against other Higgs boson modes and non-Higgs-boson SM backgrounds. The input features of the $\mathrm{WH}$ leptonic BDT are similar to those used in the $\mathrm{ZH}$ leptonic BDT, including photon, lepton, and jet kinematic variables. In addition, the transverse mass of the leading lepton and $p_{\mathrm{T}}^{\text {miss }}$ are used. The distributions of the WH leptonic BDT score for the signal and background simulation samples and data sidebands is shown figure 9 .

This single-lepton final state is sensitive to a reduced set of STXS bins. Three sets of analysis categories are defined, with $p_{\mathrm{T}}^{\gamma \gamma}$ thresholds at 75 and $150 \mathrm{GeV}$. The $p_{\mathrm{T}}^{\gamma \gamma}$ variable is used because it provides the most accurate estimate of the particle level $p_{\mathrm{T}}^{\mathrm{V}}$ used to define the STXS bins; the presence of a neutrino in the final state means that the vector boson itself cannot be fully reconstructed. The sensitivity to each set of STXS bins is optimised by deriving analysis categories based on the WH leptonic BDT score. Two analysis categories are constructed with $p_{\mathrm{T}}^{\gamma \gamma}<75 \mathrm{GeV}$ and $75<p_{\mathrm{T}}^{\gamma \gamma}<150 \mathrm{GeV}$, whilst one analysis category is defined with $p_{\mathrm{T}}^{\gamma \gamma}>150 \mathrm{GeV}$.

The analysis categories targeting $\mathrm{VH}$ production where there are no reconstructed leptons in the event are referred to as the VH MET tags. These analysis categories receive contributions from both the $\mathrm{Z}(\nu \nu) \mathrm{H}$ and $\mathrm{W}(\ell \nu) \mathrm{H}$ production modes. In addition to vetoing events with leptons, $p_{\mathrm{T}}^{\text {miss }}>50 \mathrm{GeV}$ is required and the azimuthal angle between the diphoton system and $\vec{p}_{\mathrm{T}}^{\text {miss }}$ must be greater than two radians.

With this selection the VH MET BDT is trained to discriminate between signal and background processes. The input features of the VH MET BDT rely on the same diphoton variables as in the $\mathrm{ZH}$ and $\mathrm{WH}$ leptonic BDTs, together with $p_{\mathrm{T}}^{\text {miss }}$ and jet variables. One of the dominant backgrounds in this final state consists of $\gamma+$ jets events where one of the jets is misidentified as a photon. The simulation does not model this process well and the number of such events available is limited. Hence the $\gamma+$ jets background component is modelled from a sample of data events where one of the photon candidates fails to satisfy the photon ID BDT requirement of -0.9 . To enable this, a control sample is constructed by inverting the requirement on the photon ID BDT score. These events otherwise fulfil the full set of selection requirements for the VH MET BDT channel. A new value of the photon ID BDT score is generated for each event. This is achieved by assigning a random value drawn from the photon ID BDT distribution of simulated $\gamma+$ jets events which pass the full set of selection criteria. The events are then appropriately weighted and used in the VH MET BDT training instead of the corresponding simulated samples. This is the same method first developed for the analysis described in ref. [24], but differs to the 


\begin{tabular}{|c|c|c|c|c|c|c|c|c|c|c|}
\hline \multirow{3}{*}{ Analysis categories } & \multirow{3}{*}{ Total } & \multicolumn{8}{|c|}{ SM $125 \mathrm{GeV}$ Higgs boson expected signal } & \multirow{3}{*}{$\mathrm{S} /(\mathrm{S}+\mathrm{B})$} \\
\hline & & \multirow{2}{*}{$\begin{array}{c}\text { Target } \\
\text { STXS bin(s) }\end{array}$} & \multicolumn{5}{|c|}{ Fraction of total events } & \multirow[b]{2}{*}{ Top } & \multirow{2}{*}{$\begin{array}{c}\sigma_{\text {eff }} \\
(\mathrm{GeV})\end{array}$} & \\
\hline & & & $\operatorname{ggH}$ & $\mathrm{qqH}$ & WH lep & ZH lep & ggZH lep & & & \\
\hline ZH lep Tag0 & 2.4 & $99.6 \%$ & - & - & - & $82.0 \%$ & $17.7 \%$ & $0.4 \%$ & 1.67 & 0.57 \\
\hline ZH lep Tag1 & 0.9 & $97.5 \%$ & $0.1 \%$ & - & $0.2 \%$ & $80.7 \%$ & $16.9 \%$ & $2.2 \%$ & 1.85 & 0.32 \\
\hline WH lep $p_{\mathrm{T}}^{\mathrm{V}}<75$ Tag0 & 2.0 & $81.1 \%$ & - & $0.2 \%$ & $95.0 \%$ & $3.3 \%$ & $0.2 \%$ & $1.3 \%$ & 1.89 & 0.43 \\
\hline WH lep $p_{\mathrm{T}}^{\mathrm{V}}<75$ Tag1 & 4.5 & $75.7 \%$ & $2.6 \%$ & $0.5 \%$ & $87.2 \%$ & $7.0 \%$ & $0.3 \%$ & $2.4 \%$ & 1.85 & 0.19 \\
\hline WH lep $75<p_{\mathrm{T}}^{\mathrm{V}}<150$ Tag0 & 3.0 & $77.7 \%$ & $0.7 \%$ & $0.3 \%$ & $93.2 \%$ & $3.4 \%$ & $0.8 \%$ & $1.6 \%$ & 1.94 & 0.56 \\
\hline WH lep $75<p_{\mathrm{T}}^{\mathrm{V}}<150$ Tag1 & 3.3 & $60.8 \%$ & $1.7 \%$ & $1.4 \%$ & $83.1 \%$ & $7.7 \%$ & $1.6 \%$ & $4.4 \%$ & 2.02 & 0.33 \\
\hline $\mathrm{WH}$ lep $p_{\mathrm{T}}^{\mathrm{V}}>150$ Tag0 & 3.5 & $79.9 \%$ & $0.5 \%$ & $0.4 \%$ & $91.5 \%$ & $3.6 \%$ & $1.1 \%$ & $2.8 \%$ & 1.84 & 0.77 \\
\hline VH MET Tag0 & 2.2 & $97.9 \%$ & $0.4 \%$ & $0.9 \%$ & $23.5 \%$ & $56.9 \%$ & $17.6 \%$ & $0.8 \%$ & 2.22 & 0.48 \\
\hline VH MET Tag1 & 3.6 & $90.5 \%$ & $4.6 \%$ & $3.1 \%$ & $28.8 \%$ & $46.0 \%$ & $15.7 \%$ & $1.9 \%$ & 2.30 & 0.34 \\
\hline VH MET Tag2 & 6.6 & $72.2 \%$ & $15.5 \%$ & $8.8 \%$ & $27.7 \%$ & $33.5 \%$ & $11.0 \%$ & $3.5 \%$ & 2.15 & 0.18 \\
\hline
\end{tabular}

Table 7. The expected number of signal events for $m_{\mathrm{H}}=125 \mathrm{GeV}$ in analysis categories targeting Higgs boson production in association with a leptonically decaying $\mathrm{W}$ or $\mathrm{Z}$ boson, shown for an integrated luminosity of $137 \mathrm{fb}^{-1}$. The fraction of the total number of events arising from each production mode in each analysis category is provided, as is the fraction of events originating from the targeted STXS bin or bins. Entries with values less than $0.05 \%$ are not shown. Here ggH includes contributions from the $\mathrm{ggZ}(\mathrm{q} \overline{\mathrm{q}}) \mathrm{H}$ and $\mathrm{b} \overline{\mathrm{b}} \mathrm{H}$ production modes, $\mathrm{qqH}$ incorporates both VBF and VH production with hadronic vector boson decays, and "Top" represents both $t \overline{\mathrm{t}} \mathrm{H}$ and $\mathrm{tH}$ production together. The $\sigma_{\text {eff }}$, defined as the smallest interval containing $68.3 \%$ of the $m_{\gamma \gamma}$ distribution, is listed for each analysis category. The final column shows the expected ratio of signal to signal-plus-background, $\mathrm{S} /(\mathrm{S}+\mathrm{B})$, where $\mathrm{S}$ and $\mathrm{B}$ are the numbers of expected signal and background events in a $\pm 1 \sigma_{\text {eff }}$ window centred on $m_{\mathrm{H}}$.

method used in the training of the VBF and VH hadronic BDTs. The resulting increased number of events on which to train, as well as the improved modelling of the input variable distributions, improves the performance of the VH MET BDT.

The distributions of the VH MET BDT output score for the signal and background simulation samples together with the same for the data sidebands are shown figure 9 .

The final expected signal and background yields for each ZH leptonic, WH leptonic, and VH MET analysis category are shown in table 7.

\subsection{Event categories for top quark associated production}

The coupling between the Higgs boson and the top quark affects $\mathrm{H} \rightarrow \gamma \gamma$ cross sections both via $\mathrm{ggH}$ production, entering in the gluon loop, and via decay in the diphoton decay loop. In addition, the coupling can be accessed directly by measuring the rate of $\mathrm{H} \rightarrow \gamma \gamma$ events when the Higgs boson is produced in association with one or more top quarks. The observation of $t \bar{t} \mathrm{H}$ production in the diphoton decay channel was recently reported by CMS and ATLAS [24, 64]. There, multivariate discriminants are trained separately for hadronic and leptonic decays of the top quarks to construct analysis categories enriched in $t \bar{t} \mathrm{H}$ events. In this analysis, the same techniques for the event categorisation are used. Additional analysis categories are constructed to provide sensitivity to individual STXS bins, the definitions of which are given in table 8. These correspond to the purple entries in figure 1 for $t \bar{t} \mathrm{H}$, and the single yellow entry for $\mathrm{tH}$. 


\begin{tabular}{|c|c|c|c|c|c|}
\hline \multirow[b]{2}{*}{ STXS bin } & \multirow{2}{*}{$\begin{array}{c}\text { Definition } \\
\text { units of } p_{\mathrm{T}}^{\mathrm{H}} \text { in } \mathrm{GeV}\end{array}$} & \multicolumn{3}{|c|}{ Fraction of cross section } & \multirow[b]{2}{*}{$\sigma_{\mathrm{SM}} \mathcal{B}(\mathrm{fb})$} \\
\hline & & $\mathrm{t} \overline{\mathrm{t}} \mathrm{H}$ & $\mathrm{tHq}$ & tHW & \\
\hline$t \bar{t} H$ forward & & $1.35 \%$ & - & - & 0.016 \\
\hline tH forward & $\left|y_{\mathrm{H}}\right|>2.5$ & - & $2.79 \%$ & $1.06 \%$ & 0.005 \\
\hline $\mathrm{t} \overline{\mathrm{t}} \mathrm{H} p_{\mathrm{T}}^{\mathrm{H}}<60$ & No jet requirements, $p_{\mathrm{T}}^{\mathrm{H}}<60$ & $22.42 \%$ & - & - & 0.259 \\
\hline $\mathrm{t} \overline{\mathrm{t}} \mathrm{H} 60<p_{\mathrm{T}}^{\mathrm{H}}<120$ & No jet requirements, $60<p_{\mathrm{T}}^{\mathrm{H}}<120$ & $34.61 \%$ & - & - & 0.400 \\
\hline $\mathrm{t} \overline{\mathrm{t}} \mathrm{H} 120<p_{\mathrm{T}}^{\mathrm{H}}<200$ & No jet requirements, $120<p_{\mathrm{T}}^{\mathrm{H}}<200$ & $25.60 \%$ & - & - & 0.296 \\
\hline $\mathrm{t} \overline{\mathrm{t}} \mathrm{H} 200<p_{\mathrm{T}}^{\mathrm{H}}<300$ & No jet requirements, $200<p_{\mathrm{T}}^{\mathrm{H}}<300$ & $10.72 \%$ & - & - & 0.124 \\
\hline $\mathrm{t} \overline{\mathrm{t}} \mathrm{H} p_{\mathrm{T}}^{\mathrm{H}}>300$ & No jet requirements, $p_{\mathrm{T}}^{\mathrm{H}}>300$ & $5.31 \%$ & - & - & 0.061 \\
\hline $\mathrm{tH}$ & No additional requirements & - & $97.21 \%$ & $98.94 \%$ & 0.204 \\
\hline
\end{tabular}

Table 8. Definition of the $\mathrm{t} \overline{\mathrm{t}} \mathrm{H}, \mathrm{tH}$, and $\mathrm{b} \overline{\mathrm{b}} \mathrm{H}$ STXS bins. The product of the cross section and branching fraction $(\mathcal{B})$, evaluated at $\sqrt{s}=13 \mathrm{TeV}$ and $m_{\mathrm{H}}=125 \mathrm{GeV}$, is given for each bin in the last column. The fraction of the total production mode cross section from each STXS bin is also shown. Unless stated otherwise, the STXS bins are defined for $\left|y_{\mathrm{H}}\right|<2.5$. Events with $\left|y_{\mathrm{H}}\right|>2.5$ are mostly outside of the experimental acceptance and therefore have a negligible contribution to all analysis categories.

Production of the Higgs boson in association with a single top quark is also measured in this analysis. A dedicated analysis category enriched in tHq events where the top decays leptonically is constructed. The tHq leptonic and $t \bar{t} H$ leptonic final states are very similar; an effort is therefore made to distinguish between the two.

A DNN referred to as the top DNN is trained with $t \bar{t} H$ as signal and tHq as background. It is used both by the tHq leptonic tag to reduce t $\bar{t} \mathrm{H}$ contamination, and by the $\mathrm{t} \overline{\mathrm{t}} \mathrm{H}$ leptonic analysis categories to reduce the contamination from tHq. The tHq leptonic tag is considered first in the tag priority sequence because of its lower expected signal yield. Each of the three categorisation regions ( $\mathrm{tHq}$ leptonic, $\mathrm{t} \overline{\mathrm{t}} \mathrm{H}$ leptonic, and $\mathrm{t} \overline{\mathrm{t}} \mathrm{H}$ hadronic) then uses a dedicated discriminant referred to as BDT-bkg. The purpose of the BDT-bkg is to reduce backgrounds from non-Higgs-boson SM diphoton production and split events further by expected S/B into the final analysis categories.

For an event to be considered for the tHq leptonic analysis category, it must have at least one lepton, at least one $\mathrm{b}$-tagged jet, and at least one additional jet. The top DNN and the tHq leptonic BDT-bkg are trained with these selection criteria applied. The top DNN takes both kinematic information from individual objects characteristic of top decays and global event information as inputs. The objects considered are the six leading jets and two leading leptons in $p_{\mathrm{T}}$. The four-momenta, along with the $\mathrm{b}$ tagging score and lepton identification scores, are included for each object. The global event features include the $p_{\mathrm{T}}^{\text {miss }}$, number of jets, and photon kinematic variables and identification scores.

The tHq leptonic BDT-bkg uses similar input variables to distinguish tHq events from non-Higgs boson SM backgrounds, both of which are taken from simulation to perform the training. Kinematic variables and $\mathrm{b}$ tag scores for the three leading jets and $\mathrm{b}$-tagged jets in $p_{\mathrm{T}}$ are considered, as well as photon kinematic variables, and angular variables relating the jet and photon directions. 

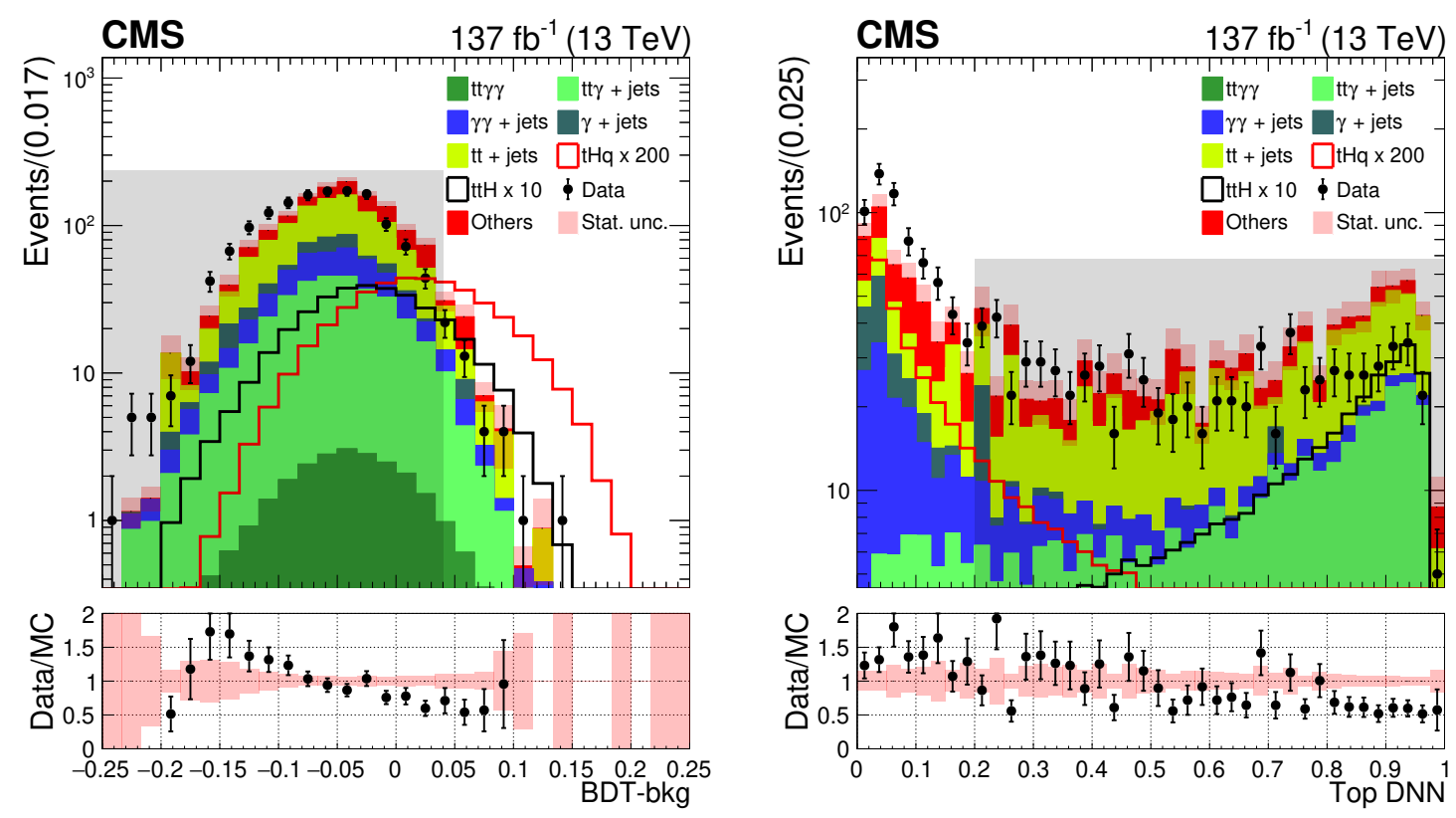

Figure 10. Distributions of tHq BDT-bkg score (left) and the top DNN (right), which are used together to define the tHq leptonic analysis category. Events are taken from the $m_{\gamma \gamma}$ sidebands, satisfying either $100<m_{\gamma \gamma}<120 \mathrm{GeV}$ or $130<m_{\gamma \gamma}<180 \mathrm{GeV}$. The statistical uncertainty in the background estimation is represented by the pink band. The regions shaded grey contain BDT-bkg and top DNN scores below and above the respective thresholds for the tHq analysis category. The full data set collected in 2016-2018 and the corresponding simulation are shown.

The distributions of the output scores for both the top DNN and the tHq BDT-bkg are shown in figure 10. In both cases, the agreement between data and simulation in this background-like region is imperfect. However, this does not affect the results of this analysis because the final background model is derived directly from data.

The final analysis category is defined by placing a requirement on both the score of the top DNN and the tHq leptonic BDT-bkg. Due to the low expected tHq signal yield, only one analysis category is constructed.

The analysis categories targeting t $\bar{t} \mathrm{H}$ production are divided into two channels, representing either fully hadronic or leptonic decays of the t $\bar{t}$ system. The hadronic channel is defined by having zero isolated leptons, whilst the leptonic channel requires one or more isolated leptons, meaning it includes events where one or both top quarks decay leptonically. In the hadronic channel, three or more jets must be present, of which at least one is tagged as originating from a bottom quark. The leptonic channel requires the presence of one or more jets, and also includes a loose requirement on the top DNN to reject tHq events. This loose preselection for both channels maximises the available number of events for the training of the BDT-bkg in each channel and the top DNN in the leptonic channel.

For each channel, the BDT-bkg is trained on simulated signal and background events. The exception is that in the hadronic channel, $\gamma+$ jets events are modelled from data. This provides both an improved description of the input features and a greater number of events 
on which to train the BDT-bkg. The procedure used to derive these events is identical to that described in section 6.4.

The inputs to the t $\bar{t} \mathrm{H}$ BDT-bkg discriminants in each channel are kinematic properties of the jets, leptons, photons, and diphoton pair. It is not possible to infer the diphoton mass from the inputs. In addition to these features, the outputs of dedicated DNNs designed to reject specific backgrounds and the output of a dedicated "top quark tagger BDT" are used [65].

The additional DNNs are trained with $t \bar{t} \mathrm{H}$ signal events against one source of background only. There are three such DNNs in total: one for each of the $\gamma \gamma+$ jets and $t \bar{t}+\gamma \gamma$ backgrounds in the hadronic channel, and one for the $t \bar{t}+\gamma \gamma$ background in the leptonic channel. These backgrounds are chosen because both they are well-modelled in simulation and because it is possible to generate a high number of simulated events on which to train. Furthermore, $t \bar{t}+\gamma \gamma$ events in particular are the principal background in the analysis categories most sensitive to t $\overline{\mathrm{t}} \mathrm{H}$ production. With these sufficiently large training samples, the background-specific DNNs are able to exploit features such as the full four-momentum vectors of physics objects. Adding these features directly to the inputs of the BDT-bkg do not improve its performance; the DNNs are required as an intermediate step to utilise this information effectively.

The top quark tagger BDT is designed to distinguish events with top quarks decaying into three jets from events that do not contain top quarks. It is trained on jet triplets from simulation of $t \bar{t}$ events, with inputs related to the kinematics, $b$ tag scores, and jet shape information. The signal is jet triplets matched at generator-level to a top quark, and background is taken as random jet triplets [65].

The output distributions of the BDT-bkg for both the hadronic and leptonic channels are shown in figure 11. To validate the modelling of the BDT-bkg in each channel, a $\mathrm{t} \overline{\mathrm{t}} \mathrm{Z}, \mathrm{Z} \rightarrow$ ee control region is used. The $\mathrm{t} \overline{\mathrm{t}} \mathrm{Z}$ events have similar kinematical properties to $t \bar{t} \mathrm{H}$ events, and are therefore suitable for testing the agreement between data and simulation in the BDT-bkg score distributions. Additional requirements on the dielectron

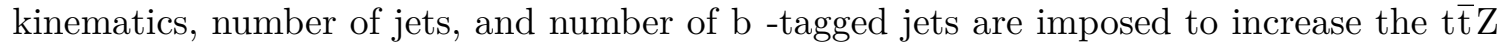
purity. The resulting comparisons between data and simulation are shown in figure 11 for the hadronic and leptonic channels.

Finally, events are split using the reconstructed $p_{\mathrm{T}}^{\gamma \gamma}$ value to targeting specific STXS bins. Analysis categories are then defined through requirements placed on the BDT-bkg output, with the boundaries chosen to maximise the expected sensitivity to each bin.

The expected signal and background yields in each analysis category targeting top quark associated Higgs boson production are shown in table 9 .

\subsection{Summary of the event categorisation}

The full set of analysis categories targeting the $\mathrm{ggH}, \mathrm{VBF}$, hadronic and leptonic $\mathrm{VH}, \mathrm{t} \overline{\mathrm{t}} \mathrm{H}$, and tHq production mechanisms are summarised in table 10. The different categorisation regions are shown in descending order of tag priority, starting with the tHq leptonic tag. If an event passes the selection criteria for more than one analysis category, it is assigned 

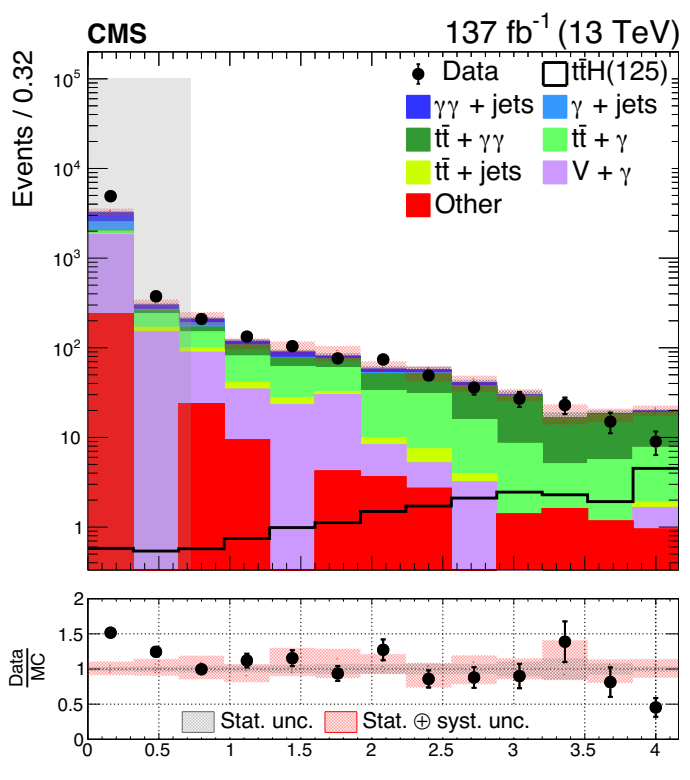

BDT-bkg
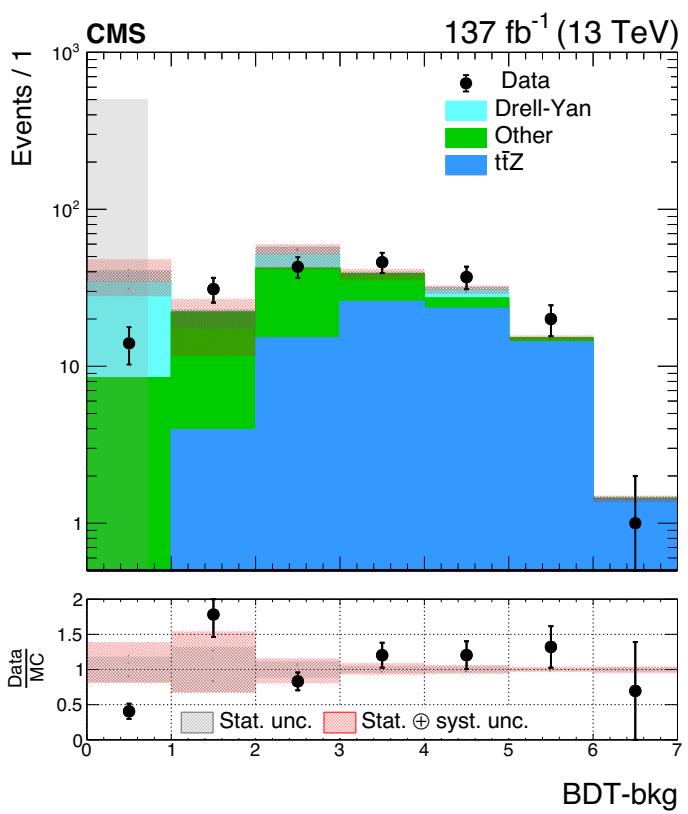
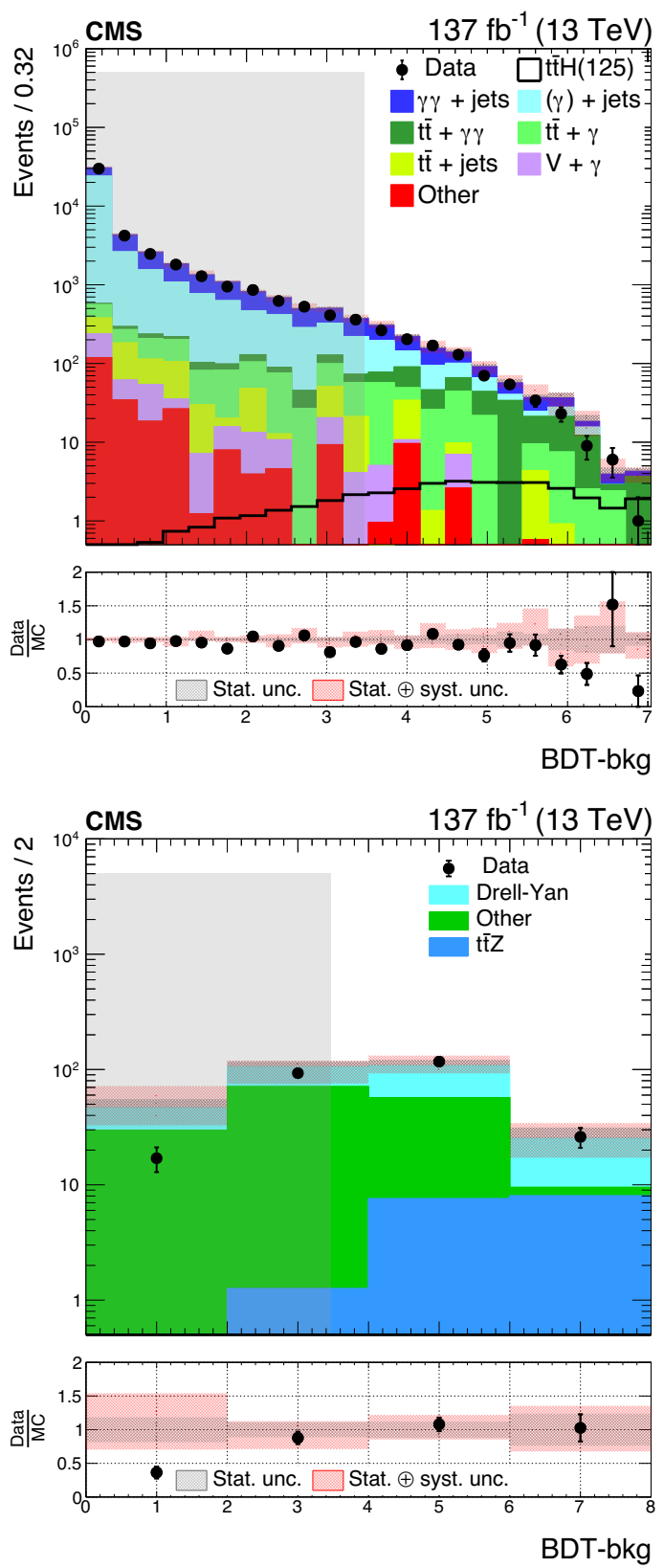

Figure 11. Distributions of BDT-bkg output used in the analysis categories targeting t $\overline{\mathrm{t}} \mathrm{H}$ production, for the leptonic (left) and the hadronic (right) channels. The upper two plots show events taken from the $m_{\gamma \gamma}$ sidebands, satisfying either $100<m_{\gamma \gamma}<120 \mathrm{GeV}$ or $130<m_{\gamma \gamma}<180 \mathrm{GeV}$. The lower two contain events from the $t \bar{t} \mathrm{Z}$ control regions, described in the text. The grey region contains BDT-bkg scores below the lowest threshold for the $t \bar{t} H$ analysis categories. Total background uncertainties (statistical $\oplus$ systematic) are represented by the black (pink) shaded bands. 


\begin{tabular}{|c|c|c|c|c|c|c|c|c|c|c|}
\hline \multirow{3}{*}{ Analysis categories } & \multirow{3}{*}{ Total } & \multicolumn{8}{|c|}{ SM $125 \mathrm{GeV}$ Higgs boson expected signal } & \multirow{3}{*}{$\mathrm{S} /(\mathrm{S}+\mathrm{B})$} \\
\hline & & \multicolumn{2}{|l|}{ Target } & \multicolumn{4}{|c|}{ Fraction of total events } & \multirow[b]{2}{*}{ tHW } & \multirow{2}{*}{$\begin{array}{c}\sigma_{\text {eff }} \\
(\mathrm{GeV})\end{array}$} & \\
\hline & & STXS bin(s) & $\operatorname{ggH}$ & $\mathrm{qqH}$ & VH lep & $t \bar{t} \mathrm{H}$ & $\mathrm{tHq}$ & & & \\
\hline tHq lep & 1.8 & $23.9 \%$ & $3.5 \%$ & $3.7 \%$ & $34.0 \%$ & $28.8 \%$ & $23.9 \%$ & $6.0 \%$ & 1.62 & 0.42 \\
\hline $\mathrm{t} \overline{\mathrm{t}} \mathrm{H}$ lep $p_{\mathrm{T}}^{\gamma \gamma}<60$ Tag0 & 0.8 & $93.8 \%$ & - & - & $0.7 \%$ & $98.2 \%$ & $0.7 \%$ & $0.5 \%$ & 1.71 & 0.72 \\
\hline $\mathrm{t} \overline{\mathrm{t}} \mathrm{H}$ lep $p_{\mathrm{T}}^{\gamma \gamma}<60$ Tag1 & 1.0 & $94.4 \%$ & - & - & $0.5 \%$ & $97.9 \%$ & $1.5 \%$ & $0.7 \%$ & 1.69 & 0.53 \\
\hline $\mathrm{t} \overline{\mathrm{t}} \mathrm{H}$ lep $p_{\mathrm{T}}^{\gamma \gamma}<60$ Tag2 & 1.8 & $87.7 \%$ & - & $0.5 \%$ & $5.1 \%$ & $90.7 \%$ & $3.2 \%$ & $1.1 \%$ & 1.94 & 0.19 \\
\hline $\mathrm{t} \overline{\mathrm{t}} \mathrm{H}$ lep $60<p_{\mathrm{T}}^{\gamma \gamma}<120$ Tag0 & 1.4 & $95.0 \%$ & - & - & $1.0 \%$ & $97.3 \%$ & $1.0 \%$ & $0.8 \%$ & 1.60 & 0.64 \\
\hline $\mathrm{t} \overline{\mathrm{t}} \mathrm{H}$ lep $60<p_{\mathrm{T}}^{\gamma \gamma}<120$ Tag1 & 0.6 & $90.8 \%$ & - & $0.7 \%$ & $1.0 \%$ & $95.6 \%$ & $1.6 \%$ & $1.1 \%$ & 1.61 & 0.55 \\
\hline tढ̄H lep $60<p_{\mathrm{T}}^{\gamma \gamma}<120$ Tag2 & 2.1 & $90.9 \%$ & - & $0.1 \%$ & $2.8 \%$ & $93.7 \%$ & $2.5 \%$ & $1.3 \%$ & 1.92 & 0.38 \\
\hline $\mathrm{t} \overline{\mathrm{t}} \mathrm{H}$ lep $120<p_{\mathrm{T}}^{\gamma \gamma}<200$ Tag0 & 3.6 & $90.1 \%$ & $0.3 \%$ & $0.2 \%$ & $2.7 \%$ & $92.8 \%$ & $2.0 \%$ & $2.0 \%$ & 1.63 & 0.71 \\
\hline $\mathrm{t} \overline{\mathrm{t}} \mathrm{H}$ lep $120<p_{\mathrm{T}}^{\gamma \gamma}<200$ Tag 1 & 0.8 & $77.9 \%$ & $2.0 \%$ & $0.5 \%$ & $11.3 \%$ & $80.6 \%$ & $3.2 \%$ & $2.5 \%$ & 1.72 & 0.43 \\
\hline $\mathrm{t} \overline{\mathrm{t}} \mathrm{H}$ lep $200<p_{\mathrm{T}}^{\gamma \gamma}<300$ Tag0 & 2.5 & $85.9 \%$ & $0.1 \%$ & - & $4.1 \%$ & $88.1 \%$ & $3.0 \%$ & $4.8 \%$ & 1.54 & 0.68 \\
\hline $\mathrm{t} \overline{\mathrm{t}} \mathrm{H}$ lep $p_{\mathrm{T}}^{\gamma \gamma}>300$ Tag0 & 2.1 & $61.7 \%$ & $1.0 \%$ & - & $18.0 \%$ & $69.3 \%$ & $3.0 \%$ & $8.7 \%$ & 1.57 & 0.69 \\
\hline $\mathrm{t} \overline{\mathrm{t}} \mathrm{H}$ had $p_{\mathrm{T}}^{\gamma \gamma}<60$ Tag 0 & 1.2 & $94.2 \%$ & $1.7 \%$ & $0.2 \%$ & - & $96.6 \%$ & $0.9 \%$ & $0.4 \%$ & 1.68 & 0.49 \\
\hline $\mathrm{t} \overline{\mathrm{t}} \mathrm{H}$ had $p_{\mathrm{T}}^{\gamma \gamma}<60$ Tag1 & 0.4 & $93.5 \%$ & $0.1 \%$ & $0.9 \%$ & - & $96.7 \%$ & $1.7 \%$ & $0.6 \%$ & 1.66 & 0.38 \\
\hline $\mathrm{t} \overline{\mathrm{t}} \mathrm{H}$ had $p_{\mathrm{T}}^{\gamma \gamma}<60$ Tag2 & 3.1 & $89.8 \%$ & $1.6 \%$ & $1.5 \%$ & $0.3 \%$ & $92.9 \%$ & $3.0 \%$ & $0.7 \%$ & 1.88 & 0.15 \\
\hline $\mathrm{t} \overline{\mathrm{t}} \mathrm{H}$ had $60<p_{\mathrm{T}}^{\gamma \gamma}<120$ Tag 0 & 1.8 & $92.6 \%$ & $0.6 \%$ & - & $0.1 \%$ & $97.6 \%$ & $1.1 \%$ & $0.6 \%$ & 1.55 & 0.77 \\
\hline $\mathrm{t} \overline{\mathrm{t}} \mathrm{H}$ had $60<p_{\mathrm{T}}^{\gamma \gamma}<120$ Tag1 & 0.4 & $90.8 \%$ & $4.6 \%$ & $0.8 \%$ & - & $91.9 \%$ & $1.9 \%$ & $0.8 \%$ & 1.35 & 0.39 \\
\hline $\mathrm{t} \overline{\mathrm{t}} \mathrm{H}$ had $60<p_{\mathrm{T}}^{\gamma \gamma}<120$ Tag2 & 5.2 & $88.7 \%$ & $1.0 \%$ & $2.2 \%$ & $0.5 \%$ & $91.8 \%$ & $3.5 \%$ & $1.0 \%$ & 1.90 & 0.23 \\
\hline $\mathrm{t} \overline{\mathrm{t}} \mathrm{H}$ had $120<p_{\mathrm{T}}^{\gamma \gamma}<200$ Tag 0 & 3.6 & $91.4 \%$ & $1.5 \%$ & $0.4 \%$ & $0.1 \%$ & $94.7 \%$ & $2.2 \%$ & $1.3 \%$ & 1.53 & 0.66 \\
\hline $\mathrm{t} \overline{\mathrm{t}} \mathrm{H}$ had $120<p_{\mathrm{T}}^{\gamma \gamma}<200$ Tag 1 & 2.1 & $83.3 \%$ & $4.6 \%$ & $2.9 \%$ & $0.5 \%$ & $86.2 \%$ & $4.2 \%$ & $1.7 \%$ & 1.76 & 0.40 \\
\hline $\mathrm{t} \overline{\mathrm{t}} \mathrm{H}$ had $120<p_{\mathrm{T}}^{\gamma \gamma}<200$ Tag2 & 1.7 & $74.3 \%$ & $10.0 \%$ & $4.6 \%$ & $0.6 \%$ & $76.5 \%$ & $6.3 \%$ & $2.0 \%$ & 1.65 & 0.29 \\
\hline $\mathrm{t} \overline{\mathrm{t}} \mathrm{H}$ had $120<p_{\mathrm{T}}^{\gamma \gamma}<200$ Tag3 & 2.6 & $62.2 \%$ & $15.4 \%$ & $8.4 \%$ & $1.2 \%$ & $64.7 \%$ & $8.5 \%$ & $1.9 \%$ & 1.73 & 0.14 \\
\hline $\mathrm{t} \overline{\mathrm{t}} \mathrm{H}$ had $200<p_{\mathrm{T}}^{\gamma \gamma}<300$ Tag 0 & 2.0 & $90.1 \%$ & $0.5 \%$ & $0.4 \%$ & $0.1 \%$ & $92.3 \%$ & $3.8 \%$ & $2.9 \%$ & 1.44 & 0.72 \\
\hline $\mathrm{t} \overline{\mathrm{t}} \mathrm{H}$ had $200<p_{\mathrm{T}}^{\gamma \gamma}<300$ Tag1 & 1.5 & $74.6 \%$ & $8.8 \%$ & $3.1 \%$ & $0.7 \%$ & $77.0 \%$ & $6.8 \%$ & $3.5 \%$ & 1.47 & 0.54 \\
\hline $\mathrm{t} \overline{\mathrm{t}} \mathrm{H}$ had $200<p_{\mathrm{T}}^{\gamma \gamma}<300$ Tag2 & 1.7 & $56.5 \%$ & $18.8 \%$ & $8.4 \%$ & $0.4 \%$ & $58.0 \%$ & $10.5 \%$ & $3.8 \%$ & 1.59 & 0.30 \\
\hline $\mathrm{t} \overline{\mathrm{t}} \mathrm{H}$ had $p_{\mathrm{T}}^{\gamma \gamma}>300$ Tag 0 & 2.5 & $73.8 \%$ & $8.3 \%$ & $1.6 \%$ & $0.8 \%$ & $74.9 \%$ & $7.7 \%$ & $6.8 \%$ & 1.44 & 0.77 \\
\hline $\mathrm{t} \overline{\mathrm{t}} \mathrm{H}$ had $p_{\mathrm{T}}^{\gamma \gamma}>300$ Tag 1 & 1.9 & $45.6 \%$ & $27.1 \%$ & $7.3 \%$ & $1.4 \%$ & $46.0 \%$ & $11.4 \%$ & $6.7 \%$ & 1.56 & 0.57 \\
\hline
\end{tabular}

Table 9. The expected number of signal events for $m_{\mathrm{H}}=125 \mathrm{GeV}$ in analysis categories targeting Higgs boson production in association with top quark, shown for an integrated luminosity of $137 \mathrm{fb}^{-1}$. The fraction of the total number of events arising from each production mode in each analysis category is provided, as is the fraction of events originating from the targeted STXS bin or bins. Entries with values less than $0.05 \%$ are not shown. Here ggH includes contributions from the $\operatorname{ggZ}(\mathrm{q} \overline{\mathrm{q}}) \mathrm{H}$ and $\mathrm{b} \overline{\mathrm{b}} \mathrm{H}$ production modes, whilst qqH incorporates both $\mathrm{VBF}$ and hadronic $\mathrm{VH}$ production. The $\sigma_{\text {eff }}$, defined as the smallest interval containing $68.3 \%$ of the $m_{\gamma \gamma}$ distribution, is listed for each analysis category. The final column shows the expected ratio of signal to signal-plusbackground, $\mathrm{S} /(\mathrm{S}+\mathrm{B})$, where $\mathrm{S}$ and $\mathrm{B}$ are the numbers of expected signal and background events in a $\pm 1 \sigma_{\text {eff }}$ window centred on $m_{\mathrm{H}}$. 
to the tag with the highest priority. Each STXS bin, or merged group of bins, and the number of analysis categories targeting it are shown.

\section{$7 \quad$ Statistical procedure}

The statistical procedure used in this analysis is identical to that described in ref. [66], as developed by the ATLAS and CMS collaborations. Simultaneous binned maximum likelihood fits are performed to the $m_{\gamma \gamma}$ distributions of all analysis categories, in the range $100<m_{\gamma \gamma}<180 \mathrm{GeV}$. A likelihood function is defined for each analysis category using analytic models to describe the $m_{\gamma \gamma}$ distributions of signal and background events, with nuisance parameters to account for the experimental and theoretical systematic uncertainties.

The analytic signal model is derived from simulation, with a model constructed for each particle level STXS bin in each reconstructed analysis category. Both the shape and normalisation of the model are parametrised as functions of $m_{\mathrm{H}}$.

The background model is determined directly from the observed $m_{\gamma \gamma}$ distribution in data. The analytic model for each analysis category can take one of a range of different functional forms, all of which represent a smoothly falling spectrum.

The best fit values and confidence intervals for the parameters of interest are estimated using a profile likelihood test statistic

$$
q(\vec{\alpha})=-2 \ln \left(\frac{L(\vec{\alpha}, \hat{\vec{\theta}} \overrightarrow{\vec{\alpha}})}{L(\hat{\vec{\alpha}}, \hat{\vec{\theta}})}\right) .
$$

The likelihood functions in the numerator and denominator of eq. (7.1) are constructed using the product over the likelihood functions defined for each analysis category. The quantities $\hat{\vec{\alpha}}$ and $\hat{\vec{\theta}}$ describe the unconditional maximum likelihood estimates for the parameters of interest and the nuisance parameters, respectively, whereas $\hat{\vec{\theta}}_{\vec{\alpha}}$ corresponds to the conditional maximum likelihood estimate for fixed values of the parameters of interest, $\vec{\alpha}$. In this analysis, the parameters of interest can be signal strengths, cross sections or coupling modifiers, depending on the fit being performed. In all fits, $m_{\mathrm{H}}$ is fixed to its most precisely measured value of $125.38 \mathrm{GeV}$ [58]. This choice is made to ensure that all measurements are reported with respect to the theoretical predictions consistent with the best available knowledge of $m_{\mathrm{H}}$. Further discussion of the implications of this choice and the difference with respect to profiling $m_{\mathrm{H}}$ is given in section 9.1.

The best fit parameter values, $\hat{\vec{\alpha}}$, are identified as those that maximise the likelihood. For one-dimensional measurements, such as the signal strength and STXS fits, the 68 and $95 \%$ confidence intervals are defined by the union of intervals for which $q(\vec{\alpha})<0.99$ and $<3.84$, respectively. In the case where there are multiple parameters of interest in the fit, the intervals are determined treating the other parameters as nuisance parameters. For two-dimensional measurements, such as those performed to coupling modifiers in the $\kappa$ framework, the 68 and $95 \%$ confidence regions are defined by the set of parameter values that satisfy $q(\vec{\alpha})<2.30$ and $<5.99$, respectively. To compute the SM expected results, 


\begin{tabular}{|c|c|c|}
\hline $\begin{array}{l}\text { Categorisation } \\
\text { region }\end{array}$ & $\begin{array}{l}\text { Particle level STXS bin, } \\
\text { (units in } \mathrm{GeV} \text { ) }\end{array}$ & $\begin{array}{l}\text { Number of } \\
\text { categories }\end{array}$ \\
\hline tHq leptonic & $\mathrm{tHq}$ & 1 \\
\hline \multirow{5}{*}{$\mathrm{t} \bar{t} \mathrm{H}$ leptonic } & $\mathrm{t} \overline{\mathrm{t}} \mathrm{H} p_{\mathrm{T}}^{\mathrm{H}}<60$ & 3 \\
\hline & $\mathrm{t} \overline{\mathrm{t}} \mathrm{H} 60<p_{\mathrm{T}}^{\mathrm{H}}<120$ & 3 \\
\hline & $\mathrm{t} \overline{\mathrm{t}} \mathrm{H} 120<p_{\mathrm{T}}^{\mathrm{H}}<200$ & 2 \\
\hline & $\mathrm{t} \overline{\mathrm{t}} \mathrm{H} 200<p_{\mathrm{T}}^{\mathrm{H}}<400$ & 1 \\
\hline & $\mathrm{t} \overline{\mathrm{t}} \mathrm{H} p_{\mathrm{T}}^{\mathrm{H}}>300$ & 1 \\
\hline \multirow{2}{*}{$\mathrm{ZH}$ leptonic } & all ZH lep and & 2 \\
\hline & ggZH lep bins (10 bins total) & 2 \\
\hline \multirow{3}{*}{ WH leptonic } & WH lep $p_{\mathrm{T}}^{\mathrm{V}}<75$ & 2 \\
\hline & all WH lep $75<p_{\mathrm{T}}^{\mathrm{V}}<150(3$ bins total $)$ & 2 \\
\hline & WH lep $p_{\mathrm{T}}^{\mathrm{V}}>150$ & 1 \\
\hline VH MET & all VH leptonic bins (15 bins total) & 3 \\
\hline \multirow{5}{*}{$\mathrm{t} \overline{\mathrm{t}} \mathrm{H}$ hadronic } & $\mathrm{t} \overline{\mathrm{t}} \mathrm{H} p_{\mathrm{T}}^{\mathrm{H}}<60$ & 3 \\
\hline & $\mathrm{t} \overline{\mathrm{t}} \mathrm{H} 60<p_{\mathrm{T}}^{\mathrm{H}}<120$ & 3 \\
\hline & $\mathrm{t} \overline{\mathrm{t}} \mathrm{H} 120<p_{\mathrm{T}}^{\mathrm{H}}<200$ & 4 \\
\hline & $\mathrm{t} \overline{\mathrm{t}} \mathrm{H} 200<p_{\mathrm{T}}^{\mathrm{H}}<400$ & 3 \\
\hline & $\mathrm{t} \overline{\mathrm{t}} \mathrm{H} p_{\mathrm{T}}^{\mathrm{H}}>300$ & 2 \\
\hline \multirow{6}{*}{ VBF } & qqH VBF-like low $m_{\mathrm{jj}}$ low $p_{\mathrm{T}}^{\mathrm{Hjj}}$ & 2 \\
\hline & $\mathrm{qqH}$ VBF-like low $m_{\mathrm{jj}}$ high $p_{\mathrm{T}}^{\mathrm{Hjj}}$ & 2 \\
\hline & qqH VBF-like high $m_{\mathrm{jj}}$ low $p_{\mathrm{T}}^{\mathrm{Hjj}}$ & 2 \\
\hline & $\mathrm{qqH}$ VBF-like high $m_{\mathrm{jj}}$ high $p_{\mathrm{T}}^{\mathrm{Hjj}}$ & 2 \\
\hline & qqH BSM & 2 \\
\hline & all $\mathrm{ggH}$ VBF-like ( 4 bins total) & 2 \\
\hline VH hadronic & qqH VH-like & 2 \\
\hline \multirow{12}{*}{$\operatorname{ggH}$} & $\operatorname{ggH} 0 \mathrm{~J}$ low $p_{\mathrm{T}}^{\mathrm{H}}$ & 3 \\
\hline & $\operatorname{ggH} 0 \mathrm{~J}$ high $p_{\mathrm{T}}^{\mathrm{H}}$ & 3 \\
\hline & $\operatorname{ggH} 1 \mathrm{~J}$ low $p_{\mathrm{T}}^{\mathrm{H}}$ & 3 \\
\hline & $\operatorname{ggH} 1 \mathrm{~J} \operatorname{med} p_{\mathrm{T}}^{\mathrm{H}}$ & 3 \\
\hline & $\operatorname{ggH} 1 \mathrm{~J}$ high $p_{\mathrm{T}}^{\mathrm{H}}$ & 3 \\
\hline & $\operatorname{ggH} \geq 2 \mathrm{~J}$ low $p_{\mathrm{T}}^{\mathrm{H}}$ & 3 \\
\hline & $\operatorname{ggH} \geq 2 \mathrm{~J} \operatorname{med} p_{\mathrm{T}}^{\mathrm{H}}$ & 3 \\
\hline & $\operatorname{ggH} \geq 2 \mathrm{~J}$ high $p_{\mathrm{T}}^{\mathrm{H}}$ & 3 \\
\hline & $\operatorname{ggH} 200<p_{\mathrm{T}}^{\mathrm{H}}<300$ & 2 \\
\hline & $\operatorname{ggH} 300<p_{\mathrm{T}}^{\mathrm{H}}<450$ & 2 \\
\hline & $\operatorname{ggH} 450<p_{\mathrm{T}}^{\mathrm{H}}<650$ & 1 \\
\hline & $\operatorname{ggH} p_{\mathrm{T}}^{\mathrm{H}}>650$ & 1 \\
\hline No categories & $\begin{array}{l}\mathrm{qqH} 0 \mathrm{~J}, 1 \mathrm{~J}, m_{\mathrm{jj}}<60,120<m_{\mathrm{jj}}<350, \\
\mathrm{~b} \overline{\mathrm{b}} \mathrm{H}, \mathrm{tHW},(6 \text { bins total })\end{array}$ & 0 \\
\hline
\end{tabular}

Table 10. Description of the different categorisation regions, listed in descending order of priority in the first column. The second column shows each targeted STXS bin, or merged group of bins, together with the number of associated analysis categories. The last row contains the bins for which no analysis categories are constructed. 
the observed data is replaced by an Asimov data set generated with all parameter values set to the SM expectation [67].

The methods used to construct the signal and background models are described in detail in the remainder of this section.

\subsection{Signal model}

The signal shape for the $m_{\gamma \gamma}$ distribution in each analysis category and for a nominal $m_{\mathrm{H}}$ is constructed from the simulation of each production process.

Since the distribution of $m_{\gamma \gamma}$ depends on whether the vertex associated with the candidate diphoton was correctly identified within $1 \mathrm{~cm}$, the correct vertex and wrong vertex scenarios are considered separately when constructing the signal model. In a given analysis category, a separate function is constructed for events originating from each STXS bin in each vertex scenario, by fitting the $m_{\gamma \gamma}$ distribution using a sum of at most five Gaussian functions. This choice provides sufficient flexibility in the fit whilst maintaining computational efficiency. The number of Gaussian functions is determined using an $\mathcal{F}$ test [68], avoiding overfitting statistical fluctuations due to the limited size of the simulated samples.

The final fit function for each analysis category is obtained by summing the individual functions for all STXS bins in both vertex scenarios. Figure 12 shows signal models for each year individually, and for the sum of the three years together. The $\sigma_{\text {eff }}$ is defined as half of the smallest interval containing $68.3 \%$ of the $m_{\gamma \gamma}$ distribution.

\subsection{Background model}

The model used to describe the background is extracted from data using the discrete profiling method [11, 69]. This technique estimates the systematic uncertainty associated with choosing a particular analytic function to fit the background $m_{\gamma \gamma}$ distribution. The choice of the background function is treated as a discrete nuisance parameter in the likelihood fit to the data.

A large set of candidate function families is considered, including exponential functions, Bernstein polynomials, Laurent series, and power law functions [69]. For each family of functions, an $\mathcal{F}$-test [68] is performed to determine the maximum order of parameters to be used, while the minimum order is determined by placing a requirement on the goodnessof-fit to the data.

When fitting these functions to the $m_{\gamma \gamma}$ distribution, the value of twice the negative logarithm of the likelihood $(-2 \Delta \ln \mathrm{L})$ is minimised. A penalty is added to $-2 \Delta \ln \mathrm{L}$ to take into account the number of floating parameters in each candidate function. When making a measurement of a given parameter of interest, the discrete profiling method minimises the overall $-2 \Delta \ln \mathrm{L}$ considering all allowed functions for each analysis category. Checks are performed to ensure that describing the background $m_{\gamma \gamma}$ distribution in this way introduces negligible bias to the final results. 

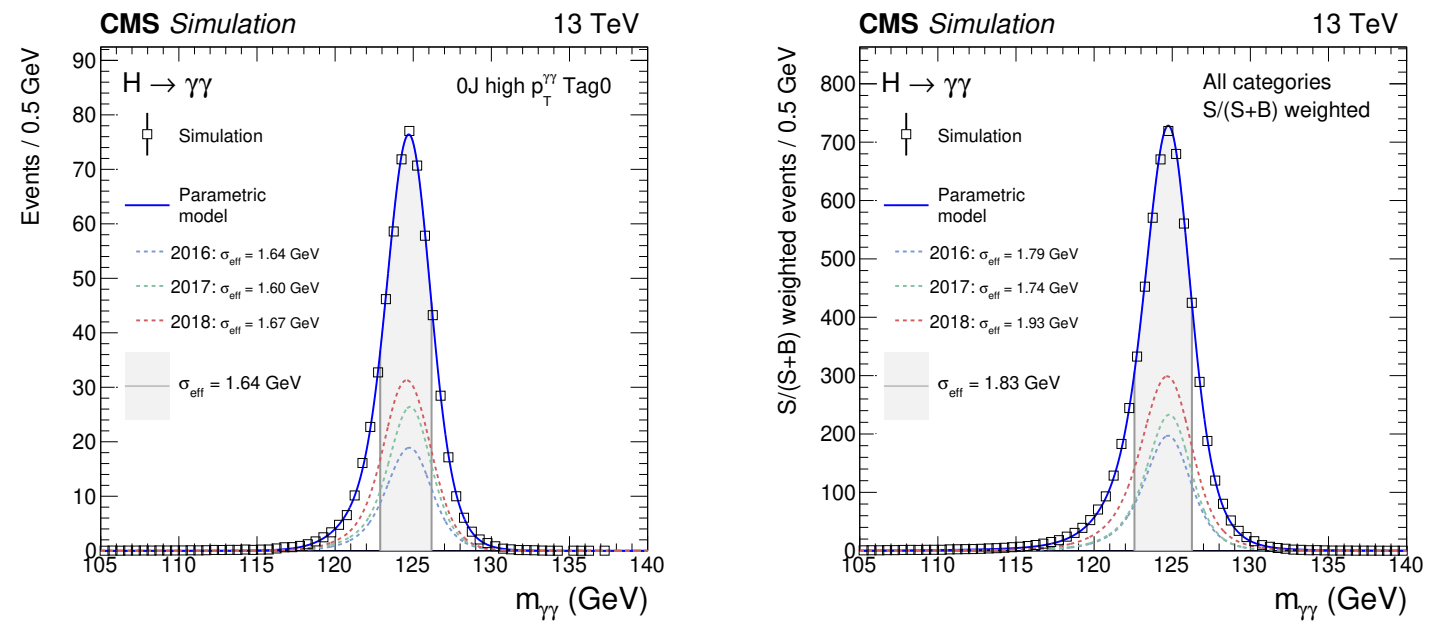

Figure 12. The shape of the parametric signal model for each year of simulated data, and for the sum of all years together, is shown. The open squares represent weighted simulation events and the blue line the corresponding model. Also shown is the $\sigma_{\text {eff }}$ value (half the width of the narrowest interval containing $68.3 \%$ of the $m_{\gamma \gamma}$ distribution) in the grey shaded area. The contribution of the signal model from each year of data taking is illustrated with the dotted lines. The models are shown for an analysis category targeting ggH $0 \mathrm{~J}$ high $p_{\mathrm{T}}^{\mathrm{H}}$ production (left), and for the weighted sum of all analysis categories (right). Here each analysis category is weighted by $\mathrm{S} /(\mathrm{S}+\mathrm{B})$, where $\mathrm{S}$ and $\mathrm{B}$ are the numbers of expected signal and background events, respectively, in a $\pm 1 \sigma_{\text {eff }} m_{\gamma \gamma}$ window centred on $m_{\mathrm{H}}$.

\section{Systematic uncertainties}

In this analysis, the systematic uncertainty associated with the background estimation from data is handled using the discrete profiling method, as described above. There are many systematic uncertainties that affect the signal model; these are handled in one of two ways. Uncertainties that modify the shape of the $m_{\gamma \gamma}$ distribution are incorporated into the signal model as nuisance parameters, where the mean and width of each Gaussian function can be affected. These uncertainties are typically experimental uncertainties relating to the energy of the individual photons. Conversely if the shape of the $m_{\gamma \gamma}$ distribution is unaffected, the uncertainty is treated as a log-normal variation in the event yield. These uncertainties include theoretical sources and experimental uncertainties such as those affecting the BDTs used to categorise events. The magnitude of each uncertainty's impact is determined individually for each STXS bin in each analysis category.

\subsection{Theoretical uncertainties}

Theoretical uncertainties affect both the overall cross section prediction for a given STXS bin, and the distributions of kinematic variables used in the event selection and categorisation. When measurements of cross sections are performed, the uncertainties in the overall cross sections are omitted, and instead are considered as uncertainties in the SM predictions. The uncertainties related to the event kinematic properties, which affect the 
efficiency and acceptance of the analysis, are still taken into account. Uncertainties affecting the overall cross section normalisations and those affecting the event kinematic properties are included when measuring signal strengths and coupling modifiers. When deriving the effect on the kinematic distributions, the impact on the STXS bin cross section normalisation is factored out to avoid double counting.

The sources of theoretical uncertainty considered in this analysis are as follows:

- Renormalisation and factorisation scale uncertainties: the uncertainty arising from variations of the renormalisation and factorisation scales used when computing the expected SM cross section and event kinematic properties. These account for the missing higher-order terms in perturbative calculations. The recommendations provided in ref. [10] are followed. The uncertainty in the overall normalisation is estimated by: varying the renormalisation by a factor of two; varying the factorisation scale by a factor of two; varying both in the same direction simultaneously. Depending on the production process, the size of the uncertainty in the overall normalisation varies from around $0.5 \%$ for $\mathrm{VBF}$ production to $15 \%$ for tHq production.

To estimate the uncertainty in the event kinematic properties, the distribution of events falling into each analysis category is recalculated when varying both the renormalisation and factorisation scales by a factor of two in the same direction simultaneously. The overall cross section for a given STXS bin is kept constant. These uncertainties, representing migrations between analysis categories, are decorrelated for different production modes and different regions of the Higgs boson phase space, resulting in 22 independent nuisance parameters. The migration uncertainties are in general around $1 \%$.

- Uncertainties in the ggH STXS fractions: for ggH production, additional sources are included that account for the uncertainty in the modelling of the $p_{\mathrm{T}}^{\mathrm{H}}$ distributions, the number of jets in the event, and the $\mathrm{ggH}$ contamination in the VBF categories. A number of sources are introduced to reflect the migration of events around the $p_{\mathrm{T}}^{\mathrm{H}}$ bin boundaries, at $10 \mathrm{GeV}$ for zero-jet events and 60 and $120 \mathrm{GeV}$ for events with at-least one jet, such that their magnitude depends on the number of jets and the $p_{\mathrm{T}}^{\mathrm{H}}$. An additional source covers the uncertainty in $p_{\mathrm{T}}^{\mathrm{H}}$ in the Lorentz-boosted region arising from the assumption of infinite top quark mass in the ggH loop. This is determined by comparing the $p_{\mathrm{T}}^{\mathrm{H}}$ distribution to the prediction from finite-mass calculations. Two further sources account for the migration between the zero, one, and two or more jet bins. The uncertainty in the ggH production of events with a VBF-like dijet system is covered by two sources corresponding to the prediction in the two- and three-jet-like bins. In addition, two nuisance parameters are introduced to account for migrations across the $m_{\mathrm{jj}}$ bin boundaries, at 350 and $700 \mathrm{GeV}$. The total magnitudes of these uncertainties vary from around 5 to $30 \%$, with events that have one or more jets and high values of $p_{\mathrm{T}}^{\mathrm{H}}$ typically having the greatest associated uncertainty. These uncertainties affect the overall cross section normalisations, and so are attributed to the SM prediction in the cross section measurements.

- Uncertainties in the qqH STXS fractions: similarly for qqH production, additional sources are introduced to account for the uncertainty in the modelling of the $p_{\mathrm{T}}^{\mathrm{H}}, m_{\mathrm{jj}}$, 
and $p_{\mathrm{T}}^{\mathrm{Hjj}}$ distributions, and the number of jets in the event. A total of six sources are defined to reflect migrations of events across $m_{\mathrm{jj}}$ boundaries at 60, 120, 350, 700, 1000 , and $1500 \mathrm{GeV}$. Two additional nuisance parameters account for migrations across the $p_{\mathrm{T}}^{\mathrm{H}}=200 \mathrm{GeV}$ and $p_{\mathrm{T}}^{\mathrm{Hjj}}=25 \mathrm{GeV}$ bin boundaries. Finally, a single source is defined to account for a migration between the zero and one, and the two or more jet bins. In each case, the uncertainty is computed by varying the renormalisation and factorisation scales and recalculating the fractional breakdown of $\mathrm{qqH}$ STXS stage- 1.2 cross sections. The total magnitude varies between bins but is at most $8 \%$. Again, these are considered as uncertainties in the SM predictions when performing cross section measurements.

- Uncertainties in the $t \bar{t} H$ STXS fractions: for $t \bar{t} H$ production, four nuisance parameters are used to account for the uncertainty in the $p_{\mathrm{T}}^{\mathrm{H}}$ distributions. Each nuisance parameter represents migration across one of the boundaries at the $p_{\mathrm{T}}^{\mathrm{H}}$ values of 60 , 120, 200, and $300 \mathrm{GeV}$ that define the ttH STXS bins. The magnitudes of these uncertainties are derived by varying the renormalisation and factorisation scales, and have values of up to $9 \%$. When performing cross section measurements, the sources are treated as uncertainties on the SM prediction.

- Uncertainties in the VH leptonic STXS fractions: for VH leptonic production, additional sources are introduced to account for the uncertainty in the modelling of the $p_{\mathrm{T}}^{\mathrm{V}}$ distributions, and the number of jets in the event. Four independent sources are defined to reflect the migrations of events across the $p_{\mathrm{T}}^{\mathrm{V}}$ boundaries at 75,150 , and $250 \mathrm{GeV}$, in addition to the migration between the zero and greater than one-jet bins for events with $p_{\mathrm{T}}^{\mathrm{V}}$ of $150-250 \mathrm{GeV}$. These sources are defined separately for the $\mathrm{WH}$ leptonic, ZH leptonic, and ggZH leptonic production modes, leading to 12 independent nuisance parameters. In each case, the uncertainty is computed by varying the renormalisation and factorisation scales and recalculating the fractional breakdown of VH leptonic STXS stage-1.2 cross sections. The total magnitude varies between bins but is at most $5 \%$ for the dominant $\mathrm{WH}$ and $\mathrm{ZH}$ leptonic production modes. Again, these are considered as uncertainties in the SM predictions when performing cross section measurements.

- Uncertainty in the ggH contamination of the top quark associated categories: the theoretical predictions for $\mathrm{ggH}$ are less reliable in a regime where the Higgs boson is produced in association with a large number of jets. Three different contributions are considered: the uncertainty from the parton shower modelling, estimated by taking the observed difference in the jet multiplicity between MADGRAPH5_aMC@NLO predictions and data in $\mathrm{t} \overline{\mathrm{t}}+$ jets events [70], the uncertainty in the gluon splitting modelling, estimated by scaling the fraction of events from $\mathrm{ggH}$ with real $\mathrm{b}$ quark jets in simulation by the measured difference between data and simulation of $\sigma(\mathrm{t} \overline{\mathrm{t}} \mathrm{b} \overline{\mathrm{b}}) / \sigma(\mathrm{t} \overline{\mathrm{t} j \mathrm{j}})[71]$ and the uncertainty due to the limited size of the simulated samples.

The combined impact of these uncertainties in the top quark associated signal strength is about $2 \%$. 
- Parton distribution function uncertainties: these account for the uncertainty due to imperfect knowledge of the composition of the proton, which affects the partons that are most likely to initiate high energy events. The overall normalisation uncertainties for each Higgs boson production process also include the uncertainty in the value of the strong coupling constant $\alpha_{\mathrm{S}}$, and are taken from ref. [10]. Uncertainties in the event kinematic properties are calculated following the PDF4LHC_100 prescription [47, 72-74] using the MC2HESSIAN procedure [75, 76]. As with the renormalisation and factorisation scale uncertainties, the normalisation for a given STXS bin is kept constant when calculating the migrations between analysis categories. The overall normalisation uncertainties are $1-5 \%$, with the migrations significantly smaller, usually less than $1 \%$.

- Uncertainty in the strong coupling constant: the uncertainty in the value of $\alpha_{\mathrm{S}}$ is included in the treatment of the PDF uncertainties, following the PDF4LHC prescription. The impact on the overall normalisation is largest for $\mathrm{ggH}$ production, with a value of $2.6 \%$. An additional source is included to account for changes in the event kinematic properties due to the uncertainty in $\alpha_{\mathrm{S}}$. This is calculated using a similar procedure to the renormalisation and factorisation scale migration uncertainties, but instead varying the value of $\alpha_{\mathrm{S}}$, and corresponds to uncertainties that are in general less than $1 \%$.

- Uncertainty in the $H \rightarrow \gamma \gamma$ branching fraction: the probability of the Higgs boson decaying to two photons is required to calculate the SM expected cross section, but this branching fraction is not known exactly. The uncertainty is currently estimated to be around 3\% [10]. This uncertainty is included in the signal strength and coupling modifier measurements, and is considered an uncertainty in the SM predictions for cross section measurements.

- Underlying-event and parton shower uncertainties: these uncertainties are obtained using dedicated simulated samples. The parton shower uncertainties originating from the modelling of the hadronization are evaluated by varying the renormalisation scale for QCD emissions in initial-state and final-state radiation by a factor of 2 and 0.5 . The uncertainties in the modelling of the underlying-event are evaluated by varying the PYTHIA8 tune from that used in the nominal simulation samples, introduced in section 4. Both these uncertainties are treated as variations in the relative contributions from each STXS bin for a given production mode, and therefore affect the STXS bin cross section normalisation. The impact is in general around $5 \%$, but can be as large as $30 \%$ for bins corresponding to high $p_{\mathrm{T}}^{\mathrm{H}}$ and high jet multiplicity.

As described in section 9.2, it is necessary to merge certain STXS bins when measuring cross sections to avoid large uncertainties or very high correlations between parameters. If two bins are measured individually, the theoretical uncertainty representing event migrations between the two bins are not included since both cross sections are being fitted. The act of merging bins across a boundary means the measurement is sensitive to the relative fraction of the two bins, and an uncertainty must be included to model this. As a result, the uncertainty sources accounting for migrations across the merged boundaries are included in the relevant cross section measurements. 


\subsection{Experimental uncertainties}

The uncertainties that affect the shape of the signal $m_{\gamma \gamma}$ distribution are listed below. These include uncertainties that account for the difference between photon showers and the electron showers used to derive the energy scale corrections. The combined effect of all signal model shape uncertainties in the measurement of the inclusive Higgs boson signal strength modifier is found to be about $2 \%$.

- Photon energy scale and resolution: the uncertainties associated with the corrections applied to the photon energy scale in data and the resolution in simulation are evaluated using $\mathrm{Z} \rightarrow$ ee events. The estimate is computed by varying the regression training scheme, the distribution of $R_{9}$, and the electron selection criteria. For the majority of photons the resulting uncertainty in the energy scale is $0.05-0.15 \%$, although for those with very high $p_{\mathrm{T}}$ the effect can be $0.5-3.0 \%$.

- Nonlinearity of the photon energy scale: a further source of uncertainty covers possible remaining differences in the linearity of the photon energy scale between data and simulation. The uncertainty is estimated using Lorentz-boosted $\mathrm{Z} \rightarrow$ ee events. In this analysis, an uncertainty of $0.2 \%$ on the photon energy scale is assigned, which accounts for the nonlinearity across the full range of photon $p_{\mathrm{T}}$ values.

- Shower shape corrections: an uncertainty in the shower shape corrections accounts for the imperfect modelling of shower shapes in simulation. The impact is estimated by comparing the energy scale before and after the corrections to shower shape variables, as described in section 5, are applied. The magnitude of the uncertainty in the energy scale ranges from $0.01-0.15 \%$, depending on the photon $|\eta|$ and $R_{9}$ values.

- Longitudinal nonuniformity of light collection: an uncertainty is associated with the modelling of the light collection as a function of emission depth within a given ECAL crystal. The calculation of this uncertainty is described in detail in ref. [58]. The uncertainty is $0.16-0.25 \%$ for photons with $R_{9}>0.96$, whilst the magnitude for low $R_{9}$ photons is below $0.07 \%$.

- Modelling of material in front of the ECAL: the amount of material through which objects pass before reaching the ECAL affects the behaviour of the electromagnetic showers, and may not be perfectly modelled in simulation. Dedicated samples with variations in the amount of upstream material are used to estimate the impact on the photon energy scale. The magnitude of the resulting uncertainty ranges from $0.02-0.05 \%$ for the most central photons, increasing to as much as $0.24 \%$ for those in the endcap.

- Vertex assignment: the largest contribution to the uncertainty in the fraction of events where the chosen vertex is smaller than $1 \mathrm{~cm}$ from the true vertex comes from the modelling of the underlying-event. In addition, the uncertainty in the ratio of data and simulation obtained using $\mathrm{Z} \rightarrow \mu^{+} \mu^{-}$events is incorporated. A nuisance parameter is included in the signal model that allows the fraction of events in each vertex scenario to vary by $\pm 2 \%$. 
The uncertainties that only modify the event yield have an effect of around $4 \%$ on the inclusive Higgs boson signal strength modifier measurement. They include the set of sources described below.

- Integrated luminosity: uncertainties of $2.5,2.3$, and $2.5 \%$ are determined by the CMS luminosity monitoring for the 2016, 2017, and 2018 data sets [38-40], respectively, whilst the uncertainty on the total integrated luminosity of the three years together is $1.8 \%$. The uncertainties for each data set are partially correlated to account for common sources in the luminosity measurement schemes.

- Photon identification BDT score: the uncertainty arising from the photon identification BDT score is estimated by varying the set of events used to train the quantile regression corrections. It is seen to cover the residual discrepancies between data and simulation. The uncertainty in the signal yields is estimated by propagating this uncertainty through the full category selection procedure. The impact in the most sensitive analysis categories is around $3 \%$.

- Jet energy scale and smearing corrections: the energy scale of jets is measured using the $p_{\mathrm{T}}$ balance of jets with $\mathrm{Z}$ bosons and photons in $\mathrm{Z} \rightarrow$ ee, $\mathrm{Z} \rightarrow \mu^{+} \mu^{-}$, and $\gamma+$ jets events, as well as the $p_{\mathrm{T}}$ balance between jets in dijet and multijet events [77]. The uncertainty in the jet energy scale is a few percent and depends on $p_{\mathrm{T}}$ and $\eta$. The impact of jet energy scale uncertainties in event yields is evaluated by varying the jet energy corrections within their uncertainties and propagating the effect to the final result. Correlations between years are introduced for the different jet energy scale uncertainty sources, ranging between 0 and $100 \%$. The impact on the category yields is largest for those targeting VBF, hadronic $\mathrm{VH}$ and top quark associated production and can be as high as $22 \%$ for the scale uncertainties, but is less than around $8 \%$ for the resolution.

- Per-photon energy resolution estimate: the uncertainty in the per-photon resolution is parametrised as a rescaling of the resolution by $\pm 5 \%$ about its nominal value. This is designed to cover all differences between data and simulation in the distribution, which is an output of the energy regression. The maximum yield variation in an analysis category is around 5\%, however for most categories the impact is below the percent level.

- Trigger efficiency: the efficiency of the trigger selection is measured with $\mathrm{Z} \rightarrow$ ee events using the tag-and-probe technique. The size of its uncertainty is less than $1 \%$. An additional uncertainty is introduced to account for a gradual shift in the timing of the inputs of the ECAL first level trigger in the region at $|\eta|>2.0$, which caused a specific trigger inefficiency during 2016 and 2017 data taking [78]. Both photons and to a greater extent jets can be affected by this inefficiency. The resulting uncertainty is largest for the categories targeting $\mathrm{VBF}$ production, with a maximum impact on the yield of $1.4 \%$.

- Photon preselection: the uncertainty in the preselection efficiency is computed as the ratio between the efficiency measured in data and in simulation. Its magnitude is less than $1 \%$. 
- Missing transverse momentum: this uncertainty is computed by shifting the reconstructed $p_{\mathrm{T}}$ of the particle candidates entering the $p_{\mathrm{T}}^{\text {miss }}$ computation, within the momentum scale and resolution uncertainties appropriate to each type of reconstructed object, as described in ref. [77]. In this analysis, the impact on the category yields is never larger than $5 \%$, even for analysis categories that explicitly use the $p_{\mathrm{T}}^{\text {miss }}$ in their definition.

- Pileup jet identification: the uncertainty in the pileup jet classification output score is estimated by comparing the score of jets in events with a $\mathrm{Z}$ boson and one balanced jet in data and simulation. The magnitude is of the order $1 \%$.

- Lepton isolation and identification: this uncertainty affecting electrons and muons is computed by varying the ratio of the efficiency in simulation to the efficiency in data and using the tag-and-probe technique in $\mathrm{Z} \rightarrow$ ee events. The resulting impact on the categories selecting leptons is up to around $1 \%$.

- $b$ jet tagging: uncertainties in the $\mathrm{b}$ tagging efficiency are evaluated by comparing data and simulated distributions for the $\mathrm{b}$ tagging discriminator. The uncertainties include the statistical component in the estimate of the fraction of heavy- and lightflavour jets in data and simulation. Its magnitude is around $3 \%$ for the analysis categories targeting top quark associated production, which make use of the $\mathrm{b}$ tagging discriminant.

Most of the experimental uncertainties are left uncorrelated among the different years. The exceptions are the partial correlations introduced for the integrated luminosity and jet energy correction uncertainties.

\section{Results}

The expected signal composition of the analysis categories in terms of a set of merged STXS bins is shown in figure 13. In the plot, the analysis categories targeting a common STXS region are summed, such that the signal compositions of the individual analysis categories are weighted according to the ratio of the numbers of signal to signal-plus-background events $(\mathrm{S} / \mathrm{S}+\mathrm{B})$. The fractional contribution of the total signal yield in a given analysis category group arising from each process is shown.

The best fit signal-plus-background model is shown with data for the sum of all analysis categories in figure 14. Again each analysis category is weighted by $(\mathrm{S} / \mathrm{S}+\mathrm{B})$, such that the absolute signal yield is kept constant.

\subsection{Signal strength modifiers}

A common signal strength modifier, $\mu$, is defined as the ratio of the observed product of the Higgs boson cross section and diphoton branching fraction to the SM expectation. It is measured to be

$$
\mu=1.12_{-0.09}^{+0.09}=1.12_{-0.06}^{+0.06}(\text { theo })_{-0.03}^{+0.03}(\text { syst })_{-0.06}^{+0.07}(\text { stat }) .
$$




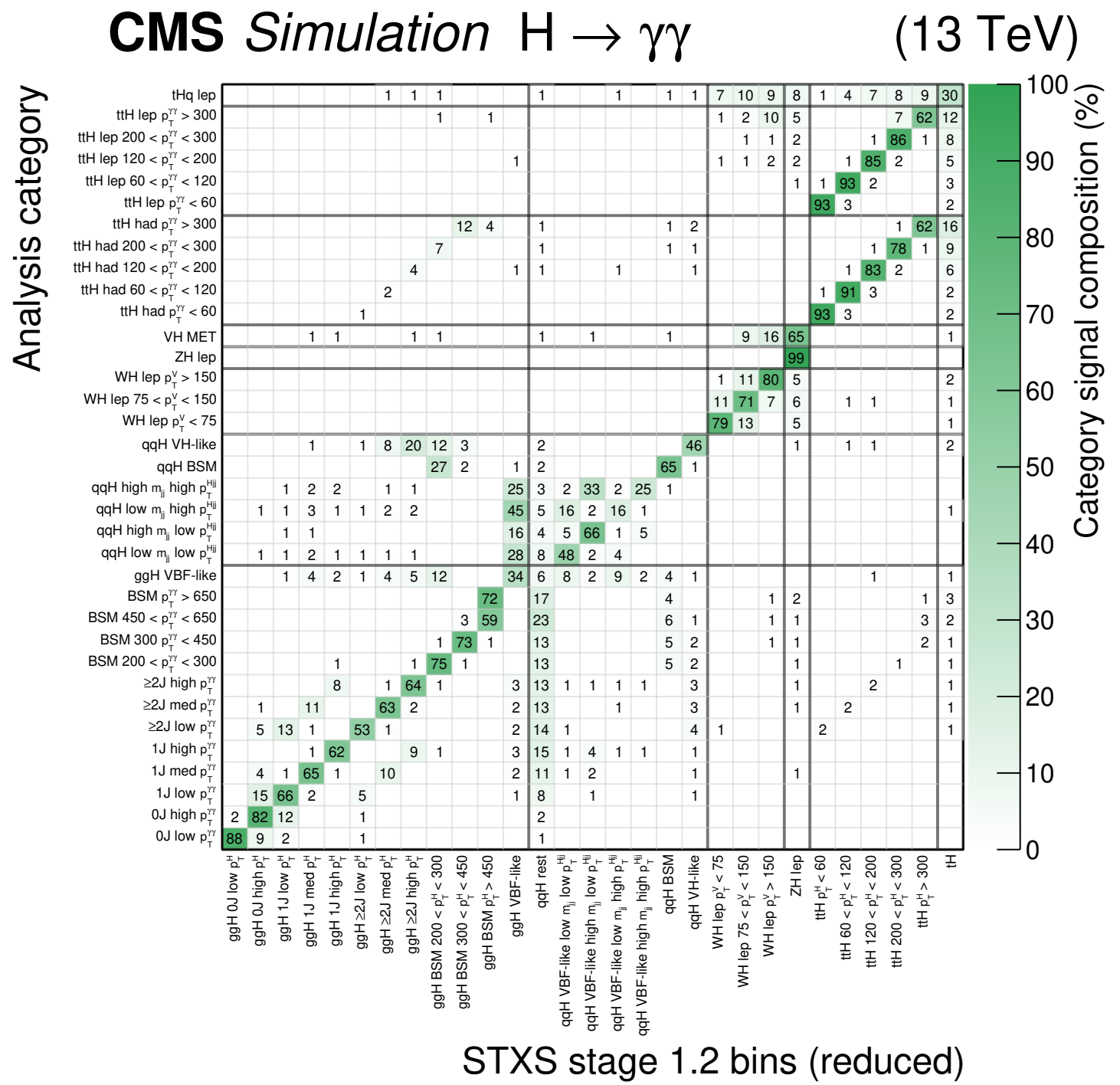

Figure 13. The composition of the analysis categories in terms of a merged set of STXS bins is shown. The granularity of the STXS bin merging corresponds to the finest granularity used for the cross section measurements in this analysis. Analysis categories targeting a common STXS region are summed, where the signal compositions of the individual categories are weighted in the sum by the expected ratio of signal to signal-plus-background events. The colour scale corresponds to the fractional yield in each analysis category group (rows) accounted for by each STXS process (columns). Each row therefore sums to $100 \%$. Entries with values less than $0.5 \%$ are not shown. Simulated events for each year in the period 2016-2018 are combined with appropriate weights corresponding to their relative integrated luminosity in data. The column labelled as "qqH rest" includes contributions from the qqH 0J, qqH $1 \mathrm{~J}$, qqH $m_{\mathrm{jj}}<60 \mathrm{GeV}$ and qqH $120<m_{\mathrm{jj}}<350 \mathrm{GeV}$ STXS bins. 

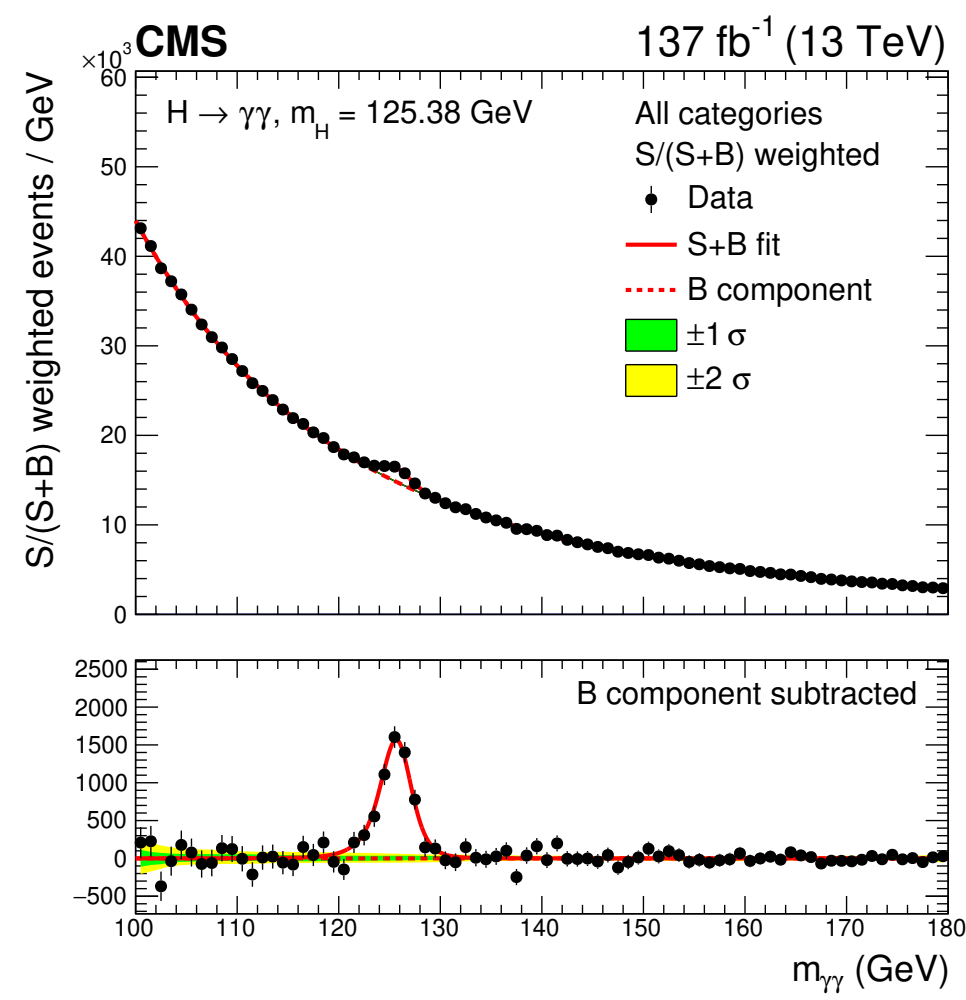

Figure 14. Data points (black) and signal-plus-background model fit for the sum of all analysis categories is shown. Each analysis category is weighted by $\mathrm{S} /(\mathrm{S}+\mathrm{B})$, where $\mathrm{S}$ and $\mathrm{B}$ are the numbers of expected signal and background events, respectively, in a $\pm 1 \sigma_{\text {eff }} m_{\gamma \gamma}$ window centred on $m_{\mathrm{H}}$. The one (green) standard deviation and two (yellow) standard deviation bands show the uncertainties in the background component of the fit. The solid red line shows the total signal-plus-background contribution, whereas the dashed red line shows the background component only. The lower panel shows the residuals after subtraction of this background component.

The uncertainty is decomposed into theoretical systematic, experimental systematic, and statistical components. The statistical component includes the uncertainty in the background modelling. The compatibility of this fit with respect to the SM prediction, expressed as a $p$-value, is approximately $17 \%$.

In this fit, and in all subsequent fits, $m_{\mathrm{H}}$ is fixed to its most precisely measured value of $125.38 \mathrm{GeV}$ [58]. The precise determination of $m_{\mathrm{H}}$ and the systematic uncertainties that enter its measurement are beyond the scope of this analysis. Nonetheless, the dependence of the measured signal strengths on $m_{\mathrm{H}}$ is checked. Profiling $m_{\mathrm{H}}$ without constraint, rather than fixing it to $125.38 \mathrm{GeV}$, has a small impact on the measured results; the best fit signal strength values change by $0.7-1.8 \%$. In each case, the change is less than $10 \%$ of the measured uncertainty.

Signal strength modifiers for each Higgs boson production mode are also measured. Unlike the subsequent STXS fits described in section 9.2, the VH hadronic and VH leptonic processes are grouped to scale according to $\mu_{\mathrm{VH}}$, whereas the VBF production mode scales with $\mu_{\mathrm{VBF}}$. The parameter $\mu_{\mathrm{top}}$ scales the $\mathrm{t} \overline{\mathrm{t}} \mathrm{H}, \mathrm{tHq}$ and tHW production modes equally and $\mu_{\mathrm{ggH}}$ scales both $\mathrm{ggH}$ and $\mathrm{b} \overline{\mathrm{b}} \mathrm{H}$ production. 
The resulting signal and background $m_{\gamma \gamma}$ distributions after the fit using this parameter scheme are shown in figure 15. Analysis categories are divided into four groups, corresponding to those targeting the $\mathrm{ggH}, \mathrm{VBF}, \mathrm{VH}$, and top quark production modes. In each group, the individual analysis categories are summed after weighting by $\mathrm{S} /(\mathrm{S}+\mathrm{B})$.

The values of the production mode signal strength modifiers and their uncertainties are displayed in figure 16. The precision of these measurements is significantly improved from previous analyses performed by the CMS collaboration in the $\mathrm{H} \rightarrow \gamma \gamma$ decay channel. In particular, the measurement of the $\mu_{\mathrm{VH}}$ signal strength modifier has improved substantially from that shown in ref. [12], beyond what would be expected from the increase in the size of the data set alone. The $p$-value of the production mode signal strength modifier fit with respect to the SM prediction is approximately $50 \%$.

The main sources of systematic uncertainty affecting the signal strength modifier in each production mode are summarised in figure 17 . The dominant contributions to the measurement uncertainty in the $\mu_{\mathrm{ggH}}, \mu_{\mathrm{VH}}$ and $\mu_{\mathrm{top}}$ signal strength modifiers originate from the corresponding renormalisation and factorisation scale uncertainties, whereas the underlying event and parton shower uncertainties are the dominant sources of uncertainty in the $\mu_{\mathrm{VBF}}$ measurement. The largest experimental uncertainties originate from the integrated luminosity, the photon identification, and the photon energy measurement for the $\mu_{\mathrm{ggH}}$ and $\mu_{\mathrm{VH}}$ signal strength modifiers. The uncertainties in the jet energy scale and resolution have a larger impact on $\mu_{\mathrm{VBF}}$ and $\mu_{\mathrm{top}}$, where $\mu_{\mathrm{top}}$ has an additional large contribution from the uncertainty in the $\mathrm{b}$ tagging.

\subsection{Simplified template cross sections}

This section details the fits performed to extract cross sections within the STXS framework and their respective $68 \%$ confidence level (CL) intervals. The theoretical uncertainties in the normalisation of the signal parameters are not included in the cross section measurements. In each fit, ggZH events in which the $\mathrm{Z}$ boson decays hadronically are grouped with the corresponding ggH STXS bin. All $b \bar{b} \mathrm{H}$ events are treated as part of the ggH 0J high $p_{\mathrm{T}}^{\mathrm{H}}$ bin. The hadronic VH processes are grouped with VBF production to form the $\mathrm{qqH}$ parameters. Parameters which are not measured are constrained to their SM prediction, within theoretical uncertainties. These are the zero jet, one jet, $m_{\mathrm{jj}}<60 \mathrm{GeV}$, and $120<m_{\mathrm{jj}}<350 \mathrm{GeV}$ bins in the $\mathrm{qqH}$ binning scheme.

Two different parameterisations are considered, with varying levels of granularity defined by the merging of certain STXS bins. It is necessary to merge bins to avoid either very large uncertainties in some parameters or very high correlations between parameters. Merging fewer bins keeps the model-dependence of the results as low as possible, as no additional assumptions are made about the relative contributions of different STXS bins. The results with reduced model-dependence however have larger uncertainties in the measured cross section parameters.

In this paper, the results of two different fits to the cross sections of partially merged STXS bins are reported. The first is referred to as the "maximal" merging scenario, where in general STXS bins are merged until their expected uncertainty is less than $150 \%$ of the SM prediction. The second "minimal" merging fit instead merges as few bins as possible 

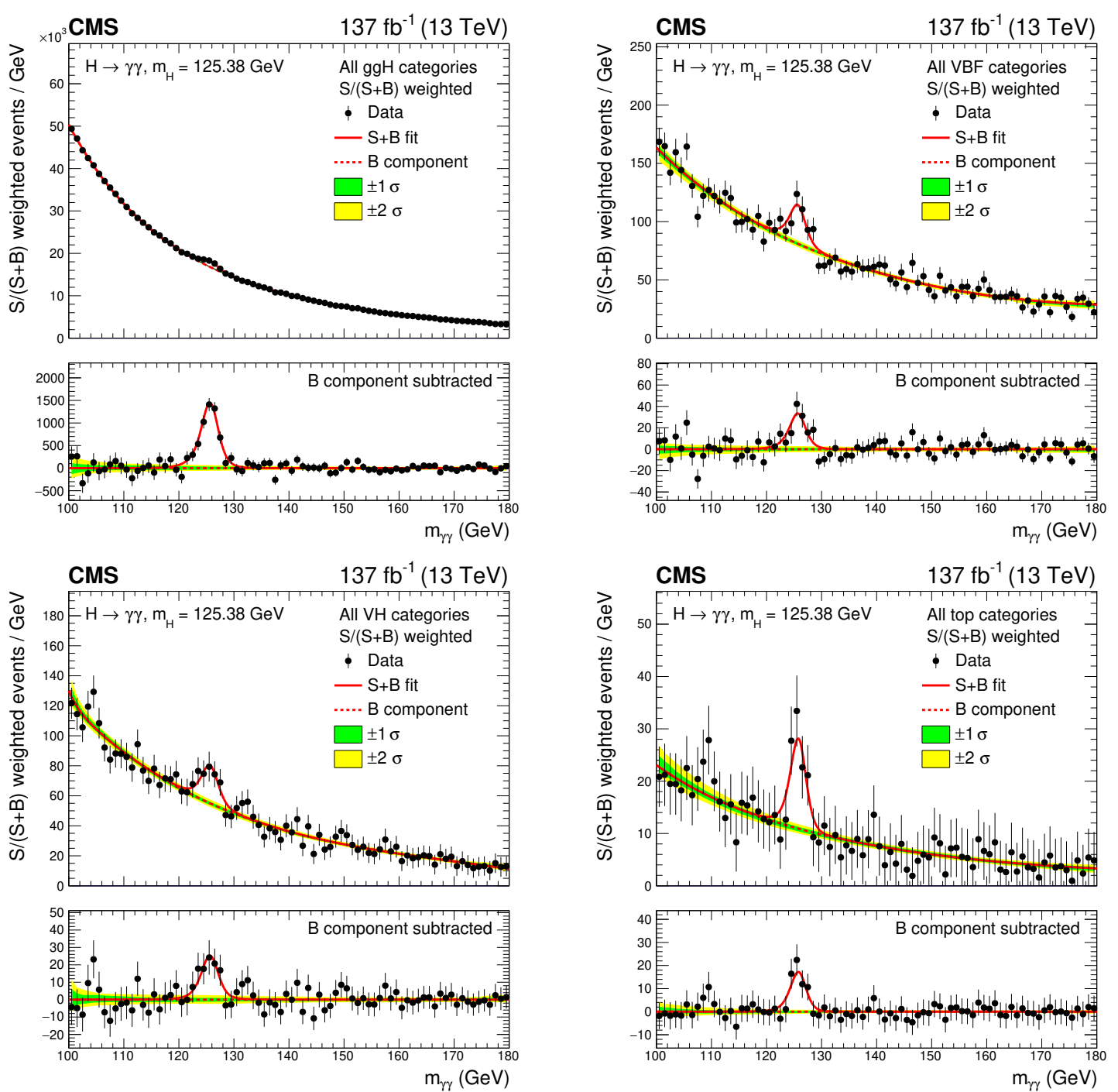

Figure 15. The best fit signal-plus-background model with data points (black) in the fit to signal strength modifiers of the four principal production modes. The model is shown separately for groups of analysis categories targeting the ggH (upper left), VBF (upper right), VH (lower left) and top quark associated (lower right) production modes. Here, the analysis categories in each group are summed after weighting by $\mathrm{S} /(\mathrm{S}+\mathrm{B})$, where $\mathrm{S}$ and $\mathrm{B}$ are the numbers of expected signal and background events in a $\pm 1 \sigma_{\text {eff }} m_{\gamma \gamma}$ window centred on $m_{\mathrm{H}}$. The one standard deviation (green) and two standard deviation (yellow) bands show the uncertainties in the background component of the fit. The solid red line shows the total signal-plus-background contribution, whereas the dashed red line represents the background component only. The lower panel in each plot shows the residuals after subtraction of this background component. 


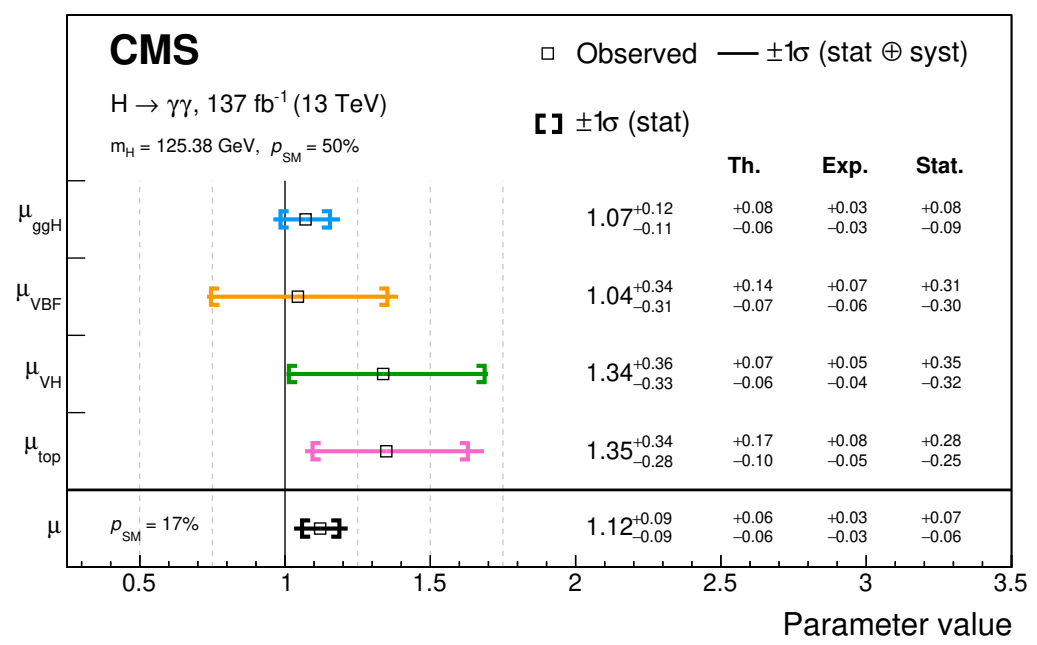

Figure 16. Observed results of the fit to signal strength modifiers of the four principal production modes. The contributions to the total uncertainty in each parameter from the theoretical systematic, experimental systematic, and statistical components are shown. The colour scheme is chosen to match the diagram presented in figure 1. The compatibility of this fit with respect to the SM prediction, expressed as a $p$-value, is approximately $50 \%$. Also shown in black is the result of the fit to the inclusive signal strength modifier, which has a $p$-value of $17 \%$.

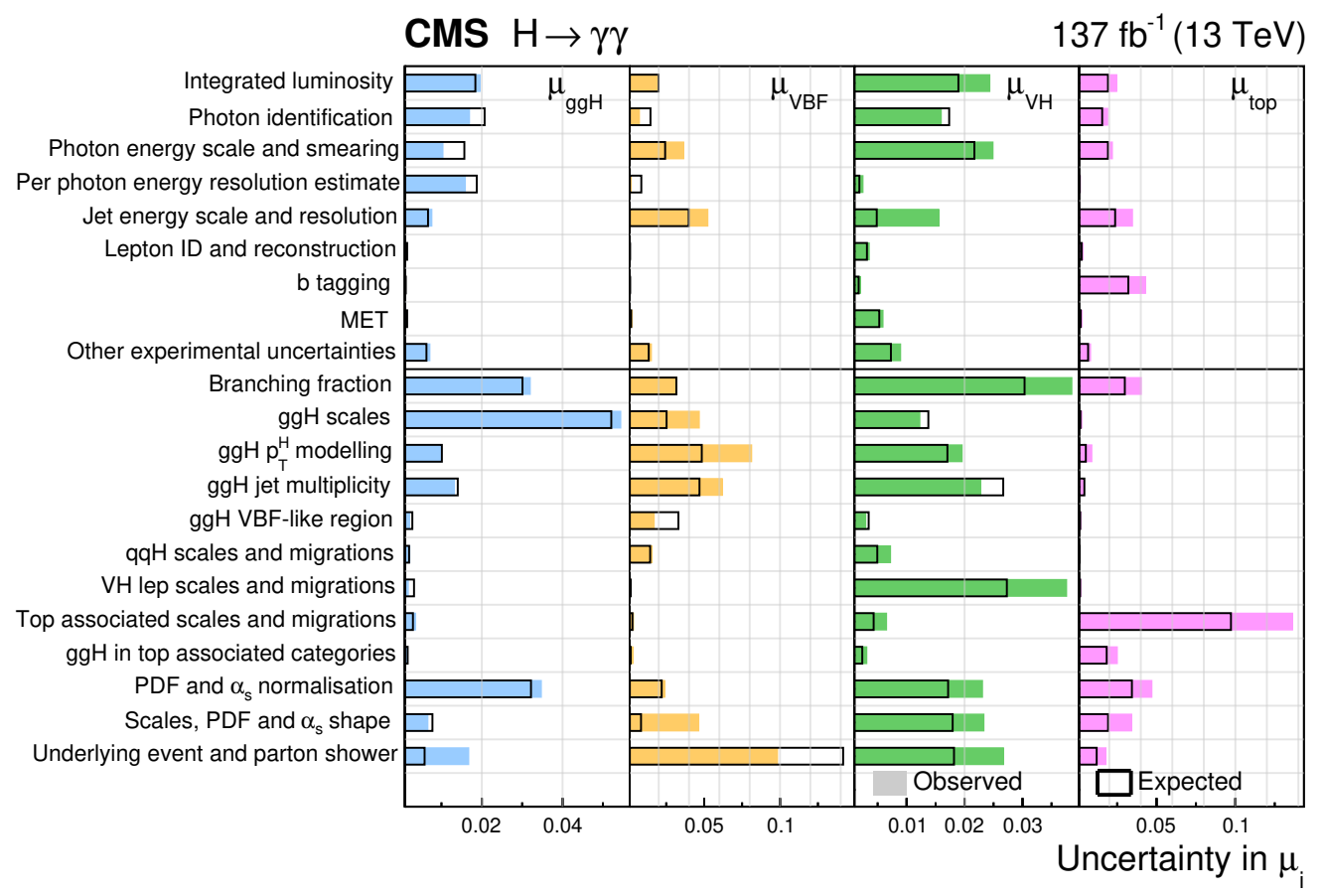

Figure 17. A summary of the impact of the main sources of systematic uncertainty in the fit to signal strength modifiers of the four principal production modes. The observed (expected) impacts are shown by the solid (empty) bars. The colour scheme is chosen to match the diagram presented in figure 1. 
whilst ensuring that parameters do not become too anti-correlated, meaning values of less than around $90 \%$.

The maximal merging scheme defines 17 parameters of interest. The VBF-like regions $\left(\geq 2\right.$-jets, $\left.m_{\mathrm{jj}}>350 \mathrm{GeV}\right)$ in the $\mathrm{ggH}$ and $\mathrm{qqH}$ schemes are merged to define the $\mathrm{ggH}$ VBF-like and qqH VBF-like parameters, respectively. The four bins with $p_{\mathrm{T}}^{\mathrm{H}}>200 \mathrm{GeV}$ in the ggH scheme are merged into a single bin, labelled as ggH BSM. Additionally, the $\mathrm{WH}$ leptonic, $\mathrm{ZH}$ leptonic and $\mathrm{t} \overline{\mathrm{t}} \mathrm{H}$ bins are all fully merged into single parameters. The $\mathrm{ZH}$ leptonic parameter groups both $\mathrm{ZH}$ and ggZH production.

The minimal merging scheme defines a more granular fit with 27 parameters of interest. The qqH VBF-like region is fully split into the four STXS bins defined by the boundaries at $m_{\mathrm{jj}}=700 \mathrm{GeV}$ and $p_{\mathrm{T}}^{\mathrm{Hjj}}=25 \mathrm{GeV}$. To avoid large correlations between the fitted parameters, the four ggH VBF-like bins are merged with the corresponding bins in the $\mathrm{qqH}$ scheme. Additional splittings are included in the ggH scheme at $p_{\mathrm{T}}^{\mathrm{H}}=300$ and $450 \mathrm{GeV}$, and the WH leptonic scheme at $p_{\mathrm{T}}^{\mathrm{V}}=75$ and $150 \mathrm{GeV}$. Furthermore, the $\mathrm{t} \overline{\mathrm{t}} \mathrm{H}$ region is split into five parameters according to the boundaries at $p_{\mathrm{T}}^{\mathrm{H}}=60,120,200$, and $300 \mathrm{GeV}$.

Table 11 summarises the maximal and minimal merging schemes by listing the STXS bins that contribute to each parameter of interest. The STXS bins that are constrained to their respective SM predictions in both fits are also listed.

The best fit cross sections and 68\% CL intervals are shown for the two merging schemes in figures 18 and 20. The corresponding numerical values are given in tables 12 and 13 . For both the maximal and minimal fits, the statistical component of the uncertainty dominates for all measured cross sections. Overall, the results from both merging scenario fits are in agreement with SM predictions; the $p$-values with respect to the SM predictions are 31 and $70 \%$ for the maximal and minimal merging scenarios, respectively.

In the maximal merging scenario, ggH production with $p_{\mathrm{T}}^{\mathrm{H}}>200 \mathrm{GeV}$, which is particularly sensitive to BSM physics entering the $\mathrm{ggH}$ loop, is measured to a precision of less than $50 \%$, relative to the SM prediction. The cross section is found to be consistent with the $\mathrm{SM}$, with a measured value of $0.9_{-0.3}^{+0.4}$ relative to the SM prediction. In addition, the product of the $\mathrm{tH}$ production cross section times $\mathrm{H} \rightarrow \gamma \gamma$ branching fraction is measured to be $1.3_{-0.7}^{+0.8} \mathrm{fb}$, corresponding to an excess of $6.3_{-3.7}^{+3.4}$ times the SM expectation. Using the $\mathrm{CL}_{\mathrm{s}}$ procedure [79], a rate of $\mathrm{tH}$ production of 14 (8) times the SM expectation is observed (expected) to be excluded at the $95 \%$ CL.

The minimal merging scenario fit represents the current most granular cross section measurement performed in a single Higgs boson decay channel, showing reasonable sensitivity to many different regions of Higgs boson production phase space. In particular, the results contain the first measurements of $t \bar{t} \mathrm{H}$ production in bins of $p_{\mathrm{T}}^{\mathrm{H}}$. The size of the uncertainty in each of the four $\mathrm{t} \overline{\mathrm{t}} \mathrm{H}$ bins with $p_{\mathrm{T}}^{\mathrm{H}}<300 \mathrm{GeV}$ is less than $100 \%$ of the $\mathrm{SM}$ prediction. Additionally, ggH production with $p_{\mathrm{T}}^{\mathrm{H}}>200 \mathrm{GeV}$ is measured in three separate regions. The three corresponding cross sections are all measured to be within one standard deviation of the respective SM expectations.

Correlations between the fitted parameters are presented in figures 19 and 21. The correlations for the ggH parameters are observed to be small between adjacent $p_{\mathrm{T}}^{\mathrm{H}}$ bins and 


\begin{tabular}{|c|c|c|}
\hline Scheme & Parameters & STXS stage 1.2 bins (total number of bins) \\
\hline \multirow{17}{*}{$\begin{array}{c}\text { Maximal } \\
(17 \text { parameters })\end{array}$} & $\operatorname{ggH} 0 \mathrm{~J}$ low $p_{\mathrm{T}}^{\mathrm{H}}$ & $\operatorname{ggH} 0 \mathrm{~J}$ low $p_{\mathrm{T}}^{\mathrm{H}}(1)$ \\
\hline & $\operatorname{ggH} 0 \mathrm{~J}$ high $p_{\mathrm{T}}^{\mathrm{H}}$ & $\operatorname{ggH} 0 \mathrm{~J}$ high $p_{\mathrm{T}}^{\mathrm{H}}, \mathrm{b} \overline{\mathrm{b}} \mathrm{H}(2)$ \\
\hline & $\operatorname{ggH} 1 \mathrm{~J}$ low $p_{\mathrm{T}}^{\mathrm{H}}$ & $\operatorname{ggH} 1 \mathrm{~J}$ low $p_{\mathrm{T}}^{\mathrm{H}}(1)$ \\
\hline & $\operatorname{ggH} 1 \mathrm{~J} \operatorname{med} p_{\mathrm{T}}^{\mathrm{H}}$ & $\operatorname{ggH} 1 \mathrm{~J} \operatorname{med} p_{\mathrm{T}}^{\mathrm{H}}(1)$ \\
\hline & $\operatorname{ggH} 1 \mathrm{~J}$ high $p_{\mathrm{T}}^{\mathrm{H}}$ & $\operatorname{ggH} 1 \mathrm{~J} \operatorname{high} p_{\mathrm{T}}^{\mathrm{H}}(1)$ \\
\hline & $\operatorname{ggH} \geq 2 \mathrm{~J}$ low $p_{\mathrm{T}}^{\mathrm{H}}$ & $\operatorname{ggH} \geq 2 \mathrm{~J}$ low $p_{\mathrm{T}}^{\mathrm{H}}(1)$ \\
\hline & $\operatorname{ggH} \geq 2 \mathrm{~J} \operatorname{med} p_{\mathrm{T}}^{\mathrm{H}}$ & $\operatorname{ggH} \geq 2 \mathrm{~J} \operatorname{med} p_{\mathrm{T}}^{\mathrm{H}}(1)$ \\
\hline & $\operatorname{ggH} \geq 2 \mathrm{~J}$ high $p_{\mathrm{T}}^{\mathrm{H}}$ & $\operatorname{ggH} \geq 2 \mathrm{~J}$ high $p_{\mathrm{T}}^{\mathrm{H}}(1)$ \\
\hline & $\operatorname{ggH} \mathrm{BSM}$ & $\left\{\begin{array}{l}\operatorname{ggH} \text { BSM } 200<p_{\mathrm{T}}^{\mathrm{H}}<300, \operatorname{ggH} \text { BSM } 300<p_{\mathrm{T}}^{\mathrm{H}}<450 \\
\operatorname{ggH} \text { BSM } 450<p_{\mathrm{T}}^{\mathrm{H}}<650, \operatorname{ggH} \text { BSM } p_{\mathrm{T}}^{\mathrm{H}}>650\end{array}\right\}$ \\
\hline & $\operatorname{ggH}$ VBF-like & $\left\{\begin{array}{l}\text { ggH VBF-like low } m_{\mathrm{jj}} \text { low } p_{\mathrm{T}}^{\mathrm{Hjj}}, \text { ggH VBF-like low } m_{\mathrm{jj}} \text { high } p_{\mathrm{T}}^{\mathrm{H} j \mathrm{j}} \\
\text { ggH VBF-like high } m_{\mathrm{jj}} \text { low } p_{\mathrm{T}}^{\mathrm{H} j \mathrm{j}}, \text { ggH VBF-like high } m_{\mathrm{jj}} \text { high } p_{\mathrm{T}}^{\mathrm{H} \mathrm{jj}}\end{array}\right\}$ (4) \\
\hline & qqH VBF-like & $\left\{\begin{array}{l}q q H \text { VBF-like low } m_{\mathrm{jj}} \text { low } p_{\mathrm{T}}^{\mathrm{Hjj}}, \text { qqH VBF-like low } m_{\mathrm{jj}} \text { high } p_{\mathrm{T}}^{\mathrm{Hjj}} \\
\text { qqH VBF-like high } m_{\mathrm{jj}} \text { low } p_{\mathrm{T}}^{\mathrm{H} j}, \mathrm{qqH} \text { VBF-like high } m_{\mathrm{jj}} \text { high } p_{\mathrm{T}}^{\mathrm{H} j \mathrm{j}}\end{array}\right\}$ (4) \\
\hline & $\mathrm{qqH}$ VH-like & qqH VH-like (1) \\
\hline & qqH BSM & qqH BSM (1) \\
\hline & WH lep & All WH lep (5) \\
\hline & ZH lep & All ZH lep and ggZH lep (10) \\
\hline & $\mathrm{t} \overline{\mathrm{t}} \mathrm{H}$ & All tētH (5) \\
\hline & $\mathrm{tH}$ & $\mathrm{tH}=\mathrm{tHq}+\mathrm{tHW}(1)$ \\
\hline \multirow{27}{*}{$\begin{array}{c}\text { Minimal } \\
(27 \text { parameters })\end{array}$} & ggH $0 \mathrm{~J}$ low $p_{\mathrm{T}}^{\mathrm{H}}$ & $\operatorname{ggH} 0 \mathrm{~J}$ low $p_{\mathrm{T}}^{\mathrm{H}}(1)$ \\
\hline & $\operatorname{ggH} 0 \mathrm{~J}$ high $p_{\mathrm{T}}^{\mathrm{H}}$ & $\operatorname{ggH} 0 \mathrm{~J} \operatorname{high} p_{\mathrm{T}}^{\mathrm{H}}, \mathrm{b} \overline{\mathrm{b}} \mathrm{H}(2)$ \\
\hline & $\operatorname{ggH} 1 \mathrm{~J}$ low $p_{\mathrm{T}}^{\mathrm{H}}$ & $\operatorname{ggH} 1 \mathrm{~J}$ low $p_{\mathrm{T}}^{\mathrm{H}}(1)$ \\
\hline & $\operatorname{ggH} 1 \mathrm{~J}$ med $p_{\mathrm{T}}^{\mathrm{H}}$ & $\operatorname{ggH} 1 \mathrm{~J}$ med $p_{\mathrm{T}}^{\mathrm{H}}(1)$ \\
\hline & $\operatorname{ggH} 1 \mathrm{~J}$ high $p_{\mathrm{T}}^{\mathrm{H}}$ & $\operatorname{ggH} 1 \mathrm{~J} \operatorname{high} p_{\mathrm{T}}^{\mathrm{H}}(1)$ \\
\hline & $\operatorname{ggH} \geq 2 \mathrm{~J}$ low $p_{\mathrm{T}}^{\mathrm{H}}$ & $\operatorname{ggH} \geq 2 \mathrm{~J}$ low $p_{\mathrm{T}}^{\mathrm{H}}(1)$ \\
\hline & $\operatorname{ggH} \geq 2 \mathrm{~J}$ med $p_{\mathrm{T}}^{\mathrm{H}}$ & $\operatorname{ggH} \geq 2 \mathrm{~J} \operatorname{med} p_{\mathrm{T}}^{\mathrm{H}}(1)$ \\
\hline & $\operatorname{ggH} \geq 2 \mathrm{~J}$ high $p_{\mathrm{T}}^{\mathrm{H}}$ & $\operatorname{ggH} \geq 2 \mathrm{~J}$ high $p_{\mathrm{T}}^{\mathrm{H}}(1)$ \\
\hline & $\operatorname{ggH}$ BSM $200<p_{\mathrm{T}}^{\mathrm{H}}<300$ & $\operatorname{ggH}$ BSM $200<p_{\mathrm{T}}^{\mathrm{H}}<300(1)$ \\
\hline & $\operatorname{ggH}$ BSM $300<p_{\mathrm{T}}^{\mathrm{H}}<450$ & $\operatorname{ggH}$ BSM $300<p_{\mathrm{T}}^{\mathrm{H}}<450(1)$ \\
\hline & $\operatorname{ggH} \operatorname{BSM} p_{\mathrm{T}}^{\mathrm{H}}>450$ & $\operatorname{ggH}$ BSM $450<p_{\mathrm{T}}^{\mathrm{H}}<650, \operatorname{ggH} \mathrm{BSM} p_{\mathrm{T}}^{\mathrm{H}}>650(2)$ \\
\hline & VBF-like low $m_{\mathrm{jj}}$ low $p_{\mathrm{T}}^{\mathrm{H} \mathrm{jj}}$ & $\operatorname{ggH}+\mathrm{qqH}$ VBF-like low $m_{\mathrm{jj}}$ low $p_{\mathrm{T}}^{\mathrm{H} j \mathrm{j}}(2)$ \\
\hline & VBF-like low $m_{\mathrm{jj}}$ high $p_{\mathrm{T}}^{\mathrm{H} j \mathrm{j}}$ & ggH + qqH VBF-like low $m_{\mathrm{jj}}$ high $p_{\mathrm{T}}^{\mathrm{H} j \mathrm{j}}(2)$ \\
\hline & VBF-like high $m_{\mathrm{jj}}$ low $p_{\mathrm{T}}^{\mathrm{Hjj}}$ & ggH + qqH VBF-like high $m_{\mathrm{jj}}$ low $p_{\mathrm{T}}^{\mathrm{Hjj}}(2)$ \\
\hline & VBF-like high $m_{\mathrm{jj}}$ high $p_{\mathrm{T}}^{\mathrm{H} j \mathrm{j}}$ & $\operatorname{ggH}+\mathrm{qqH}$ VBF-like high $m_{\mathrm{jj}}$ high $p_{\mathrm{T}}^{\mathrm{H} j \mathrm{j}}(2)$ \\
\hline & qqH VH-like & qqH VH-like (1) \\
\hline & $\mathrm{qqH} \mathrm{BSM}$ & qqH BSM (1) \\
\hline & WH lep $p_{\mathrm{T}}^{\mathrm{V}}<75$ & WH lep $p_{\mathrm{T}}^{\mathrm{V}}<75(1)$ \\
\hline & WH lep $75<p_{\mathrm{T}}^{\mathrm{V}}<150$ & WH lep $75<p_{\mathrm{T}}^{\mathrm{V}}<150(1)$ \\
\hline & WH lep $p_{\mathrm{T}}^{\mathrm{V}}>150$ & $\left\{\begin{array}{l}\text { WH lep 0J } 150<p_{\mathrm{T}}^{\mathrm{V}}<250, \mathrm{WH} \text { lep } \geq 1 \mathrm{~J} 150<p_{\mathrm{T}}^{\mathrm{V}}<250 \\
\mathrm{WH} \text { lep } p_{\mathrm{T}}^{\mathrm{V}}>250\end{array}\right\}$ \\
\hline & ZH lep & All ZH lep and ggZH lep (10) \\
\hline & $\mathrm{t} \overline{\mathrm{t}} \mathrm{H} p_{\mathrm{T}}^{\mathrm{H}}<60$ & $\mathrm{t} \overline{\mathrm{t}} \mathrm{H} p_{\mathrm{T}}^{\mathrm{H}}<60(1)$ \\
\hline & $\mathrm{t} \overline{\mathrm{t}} \mathrm{H} 60<p_{\mathrm{T}}^{\mathrm{H}}<120$ & $\mathrm{t} \overline{\mathrm{t}} \mathrm{H} 60<p_{\mathrm{T}}^{\mathrm{H}}<120(1)$ \\
\hline & $\mathrm{t} \overline{\mathrm{t}} \mathrm{H} 120<p_{\mathrm{T}}^{\mathrm{H}}<200$ & $\mathrm{t} \overline{\mathrm{t}} \mathrm{H} 120<p_{\mathrm{T}}^{\mathrm{H}}<200(1)$ \\
\hline & $\mathrm{t} \overline{\mathrm{t}} \mathrm{H} 200<p_{\mathrm{T}}^{\mathrm{H}}<300$ & $\mathrm{t} \overline{\mathrm{t}} \mathrm{H} 200<p_{\mathrm{T}}^{\mathrm{H}}<300(1)$ \\
\hline & $\mathrm{t} \overline{\mathrm{t}} \mathrm{H} p_{\mathrm{T}}^{\mathrm{H}}>300$ & $\mathrm{t} \overline{\mathrm{t}} \mathrm{H} p_{\mathrm{T}}^{\mathrm{H}}>300(1)$ \\
\hline & $\mathrm{tH}$ & $\mathrm{tH}=\mathrm{tHq}+\mathrm{tHW}(1)$ \\
\hline \multicolumn{2}{|c|}{ Constrained to SM prediction } & qqH $0 J, \mathrm{qqH} 1 \mathrm{~J}, \mathrm{qqH} m_{\mathrm{jj}}<60, \mathrm{qqH} 120<m_{\mathrm{jj}}<350(4)$ \\
\hline
\end{tabular}

Table 11. A summary of the maximal and minimal parameter merging scenarios. The STXS bins that contribute to each parameter are listed. Furthermore, the bins that are constrained to their respective SM predictions in the fits are listed in the final row. 


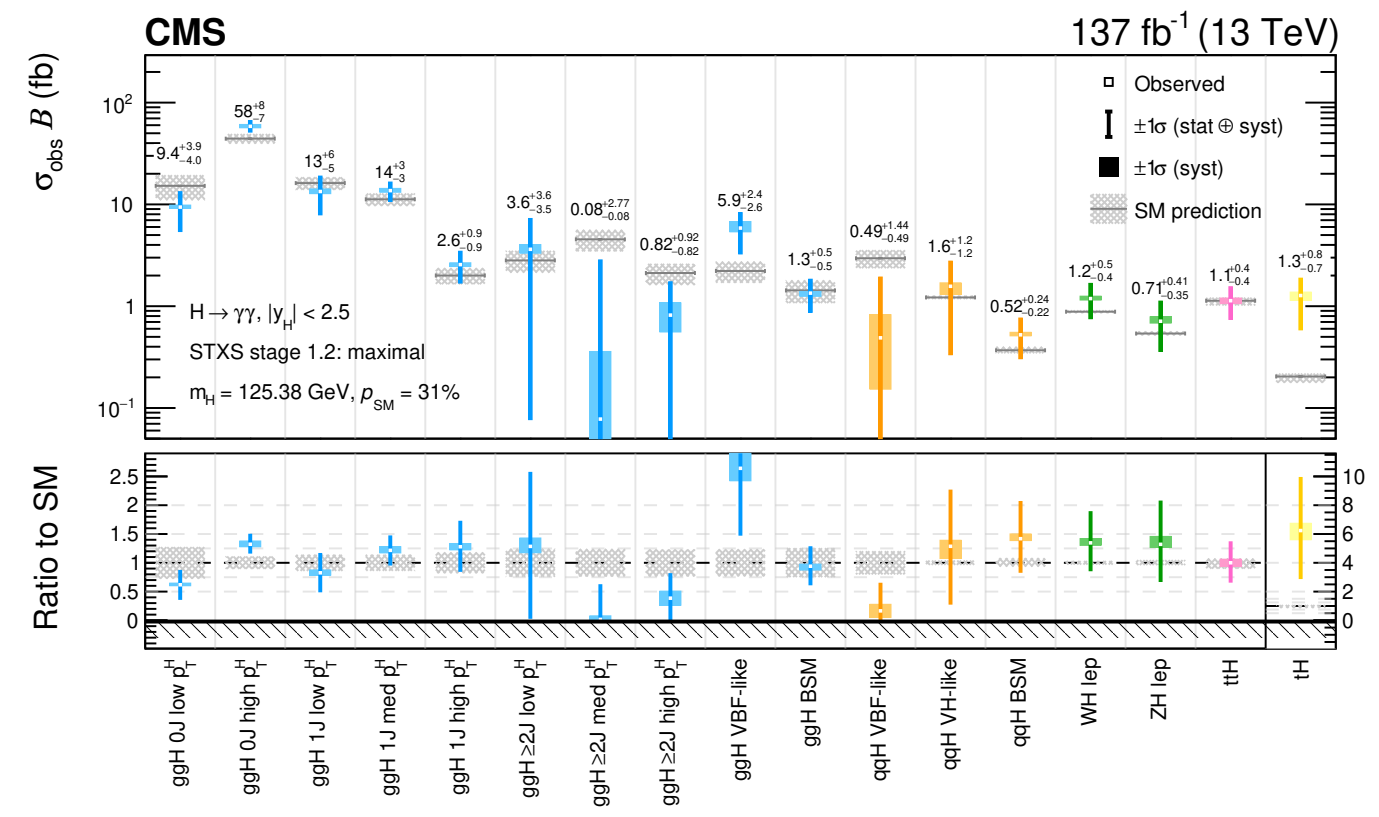

Figure 18. Observed results of the maximal merging scheme STXS fit. The best fit cross sections are plotted together with the respective $68 \% \mathrm{CL}$ intervals. The systematic components of the uncertainty in each parameter are shown by the coloured boxes. The hatched grey boxes demonstrate the theoretical uncertainties in the SM predictions. The lower panel shows the ratio of the fitted values to the SM predictions. Here the tH cross section ratio has a different scale, due to its high best fit value and uncertainty. The cross sections are constrained to be non-negative, as indicated by the hashed pattern below zero. The parameters whose best fit values are at zero are known to have $68 \%$ CL intervals which slightly under-cover; this is checked to be a small effect using pseudo-experiments. The colour scheme is chosen to match the diagram presented in figure 1. The compatibility of this fit with respect to the SM prediction, expressed as a $p$-value, is approximately $31 \%$.

larger between adjacent number of jet bins. This results from the fact that $p_{\mathrm{T}}^{\gamma \gamma}$ is a wellmeasured quantity, whereas reconstructing the number of jets in an event is more difficult. Nevertheless, the application of the ggH BDT in the event categorisation helps to minimise these correlations. The largest correlations in the maximal merging scheme exist between the $\mathrm{ggH}$ VBF-like and qqH VBF-like cross sections and the $\mathrm{t}_{\overline{\mathrm{t}} \mathrm{H}}$ and $\mathrm{tH}$ cross sections, with values of -0.76 and -0.59 , respectively. These result from the sizeable contamination of $\mathrm{ggH}$ VBF-like events in the $\mathrm{qqH}$ analysis categories, and the contamination of $\mathrm{t} \overline{\mathrm{t}} \mathrm{H}$ events in the $\mathrm{tHq}$ leptonic category. The act of splitting $\mathrm{t} \overline{\mathrm{t}} \mathrm{H}$ production into five separate parameters in the minimal merging scenario introduces larger correlations into the measurement.

\subsection{Coupling modifiers}

The $\kappa$-framework defines coupling modifiers to directly parametrise deviations from the SM expectation in the couplings of the Higgs boson to other particles [14]. Two different likelihood scans, each with two dimensions, are performed. Full details of each parameterisation are given in ref. [8]. 


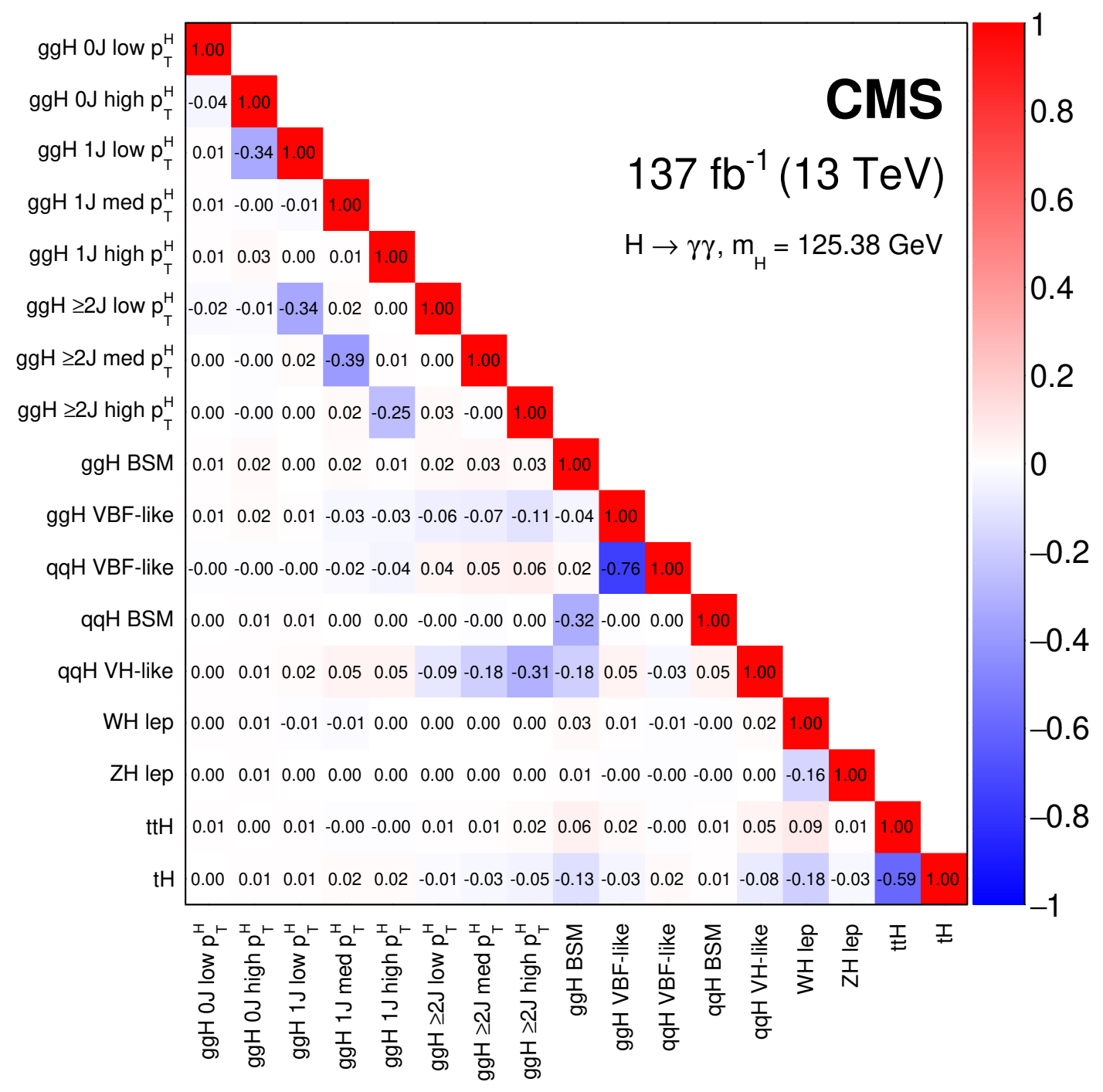

Figure 19. Observed correlations between the 17 parameters considered in the maximal merging STXS fit. The size of the correlations is indicated by the colour scale.

In the first fit, the resolved $\kappa$ model is used. Here the scaling factors of loops present in Higgs boson production and decay are resolved into their SM components, in terms of the other $\kappa$ parameters. The most important of these are in $\mathrm{ggH}$ production and $\mathrm{H} \rightarrow \gamma \gamma$ decay, but others, such as the loop in ggZH production, are also resolved. The results of a two-dimensional scan in $\kappa_{\mathrm{V}}$ and $\kappa_{\mathrm{F}}$, scaling the Higgs boson coupling to vector bosons and to fermions, respectively, are shown in the upper plot of figure 22. The $\mathrm{H} \rightarrow \gamma \gamma$ decay rate contains an interference term proportional to $\kappa_{\mathrm{V}} \kappa_{\mathrm{F}}$. This means that the rate of $g \mathrm{gH}$ and $\mathrm{t} \overline{\mathrm{t}} \mathrm{H}$ production $\left(\propto \kappa_{\mathrm{F}}^{2}\right)$, relative to the rate of $\mathrm{VBF}$ and $\mathrm{VH}$ production $\left(\propto \kappa_{\mathrm{V}}^{2}\right)$, can be used to gain sensitivity to the relative sign of the tt-H and VV-H couplings. In addition, the tHq and tHW production modes also include a term proportional to $\kappa_{\mathrm{V}} \kappa_{\mathrm{F}}$. This analysis explicitly targets tHq production via the tHq leptonic analysis category, the inclusion of which helps to further reduce the degeneracy between positive and negative $\kappa_{\mathrm{F}}$ values. The 


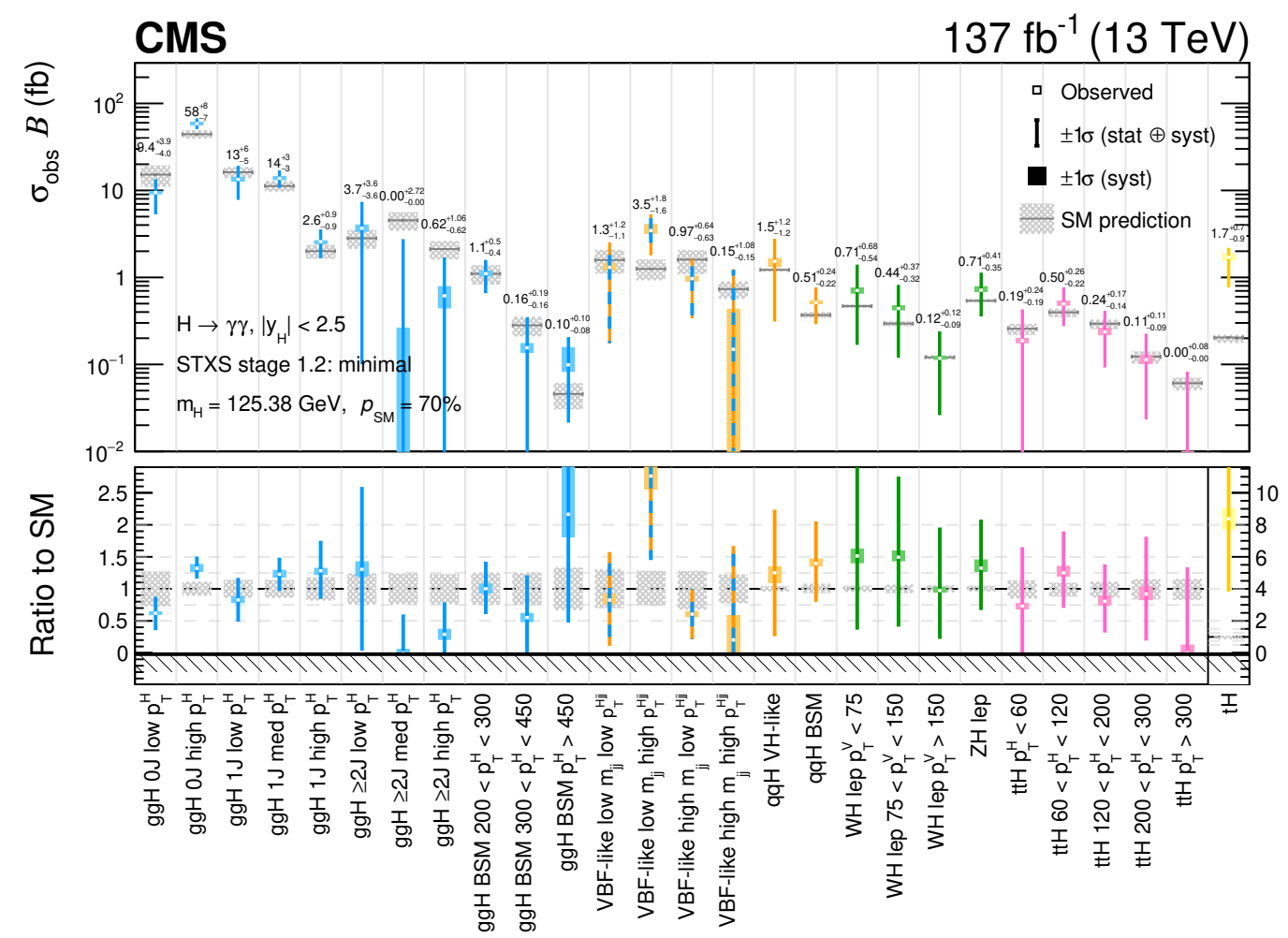

Figure 20. Observed results of the minimal merging scheme STXS fit. The best fit cross sections are plotted together with the respective $68 \% \mathrm{CL}$ intervals. The systematic components of the uncertainty in each parameter are shown by the coloured boxes. The hatched grey boxes demonstrate the theoretical uncertainties in the SM predictions. The lower panel shows the ratio of the fitted values to the SM predictions. Here the $\mathrm{tH}$ cross section ratio has a different scale, due to its high best fit value and uncertainty. The cross sections are constrained to be non-negative, as indicated by the hashed pattern below zero. The parameters whose best fit values are at zero are known to have $68 \%$ CL intervals which slightly under-cover; this is checked to be a small effect using pseudoexperiments. The colour scheme is chosen to match the diagram presented in figure 1 . The orange lines dashed with blue for the VBF-like parameters represent contributions from both the ggH and the qqH STXS bins. The compatibility of this fit with respect to the SM prediction, expressed as a $p$-value, is approximately $70 \%$.

region with negative values of $\kappa_{\mathrm{F}}$ is observed (expected) to be excluded with a significance of 0.5 (2.4) standard deviations. The reduction in the observed significance with respect to the expected is due to the observed excess in the $\mathrm{tH}$ production mode cross section.

A second fit is performed using the unresolved $\kappa$ model, where the $\mathrm{ggH}$ and $\mathrm{H} \rightarrow \gamma \gamma$ loops are given their own effective scaling factors denoted $\kappa_{\mathrm{g}}$ and $\kappa_{\gamma}$, respectively. The $\kappa_{\mathrm{g}}$ and $\kappa_{\gamma}$ parameters are particularly sensitive to additional BSM states, that contribute towards the rate of Higgs boson production and decay via loop processes. The observed result of a two-dimensional scan in these two parameters is shown in the lower plot of figure 22. In the scan, the other $\kappa$ parameters in the unresolved model are fixed to unity. The best fit point is consistent with the SM expectation at approximately the $68 \%$ CL. 


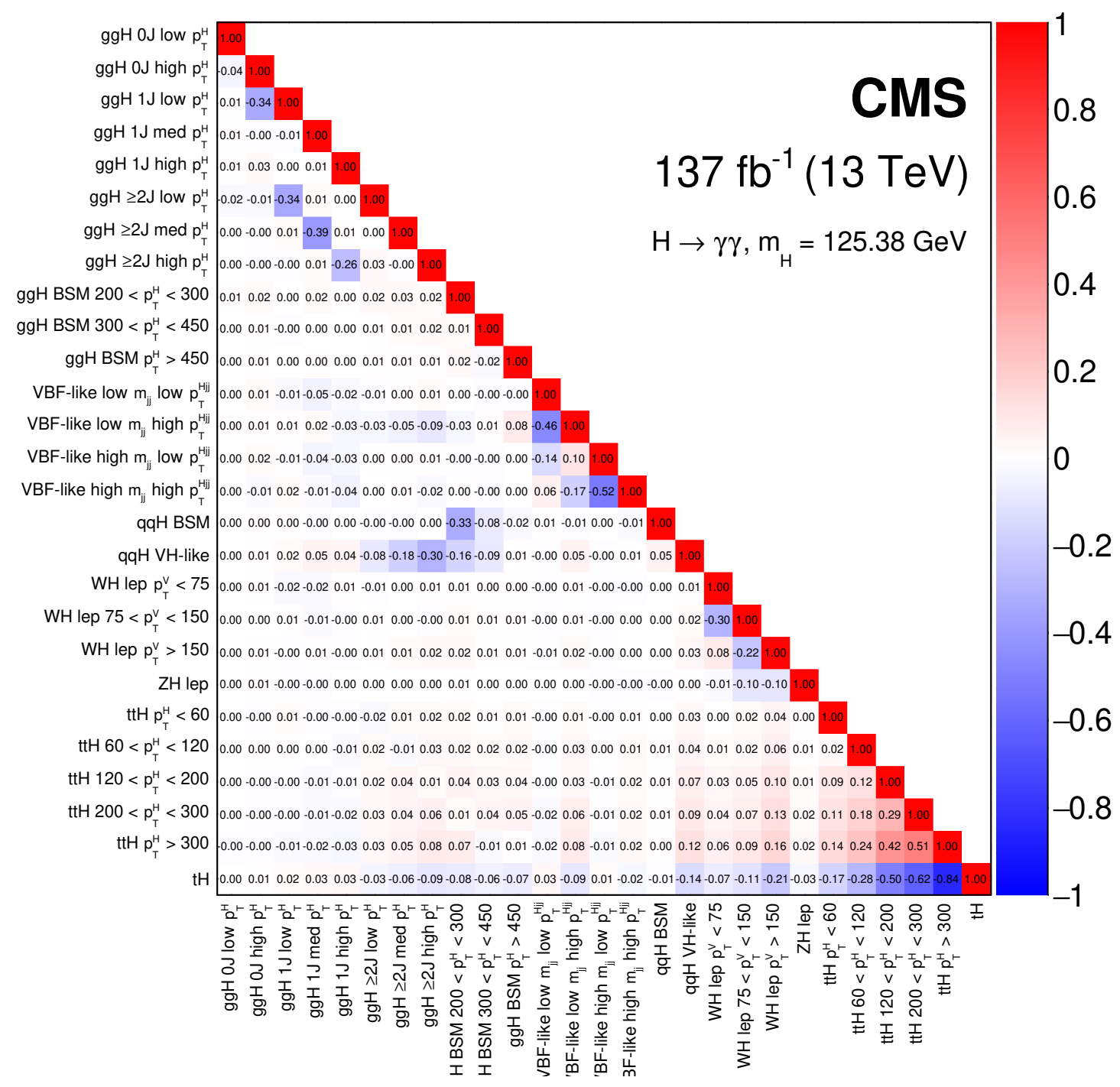

Figure 21. Observed correlations between the 27 parameters considered in the minimal merging STXS fit. The size of the correlations is indicated by the colour scale.

\section{Summary}

Measurements of Higgs boson properties with the Higgs boson decaying into a pair of photons are reported. Events with two photons are selected from a sample of protonproton collisions at a centre-of-mass energy $\sqrt{s}=13 \mathrm{TeV}$ collected with the CMS detector at the LHC from 2016 to 2018, corresponding to an integrated luminosity of $137 \mathrm{fb}^{-1}$. Analysis categories enriched in events produced via gluon fusion, vector boson fusion, vector boson associated production, production associated with two top quarks, and production associated with one top quark are constructed.

A range of production and coupling properties of the Higgs boson are measured. The total Higgs boson signal strength, relative to the standard model (SM) prediction, is measured to be $1.12 \pm 0.09$. A simultaneous measurement of the signal strengths of the four 

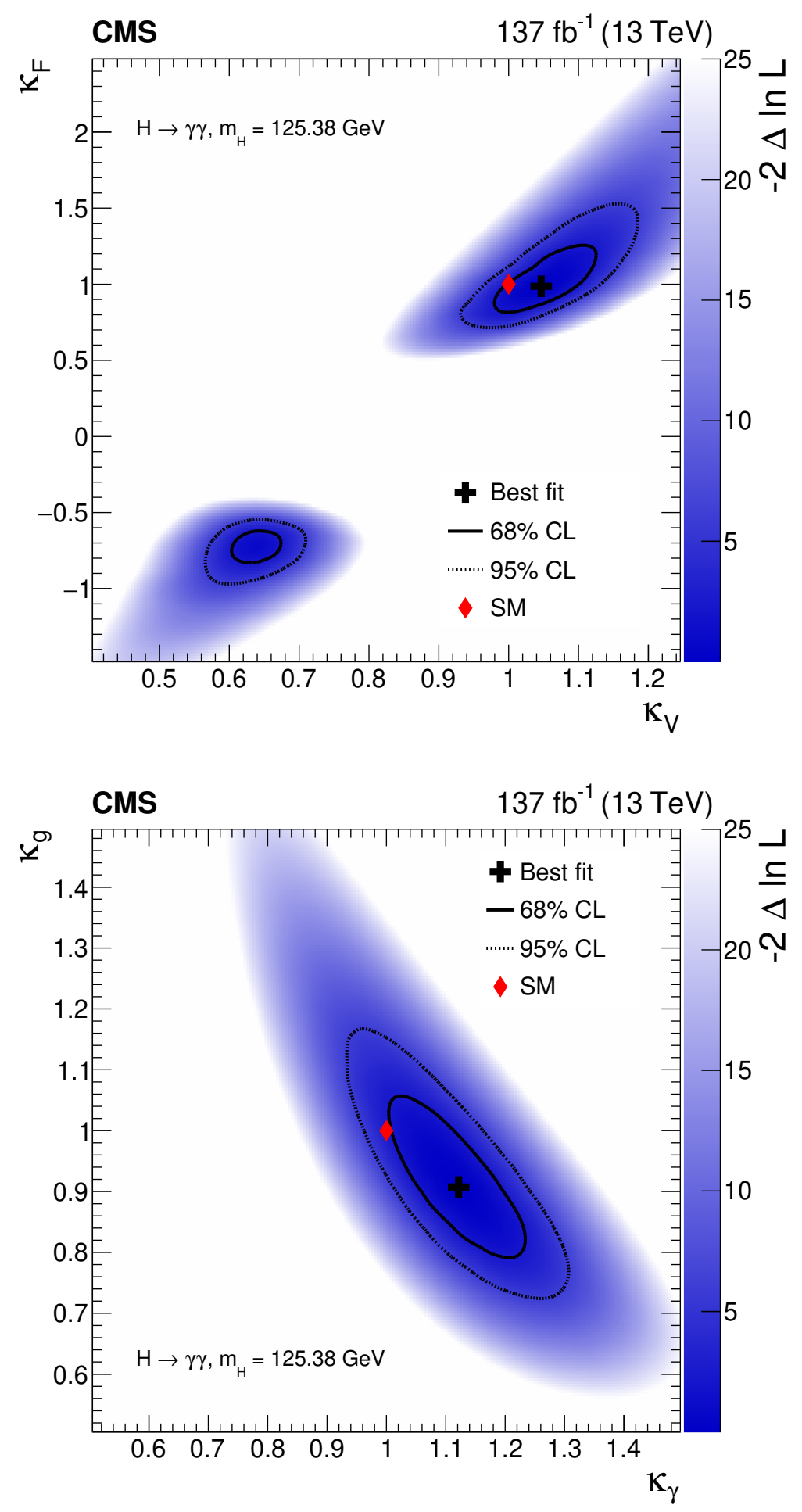

Figure 22. Observed two-dimensional likelihood scans performed in the $\kappa$-framework: $\kappa_{\mathrm{V}}$-vs- $\kappa_{\mathrm{F}}$ in the resolved $\kappa$ model (upper) and $\kappa_{\gamma}$-vs- $\kappa_{\mathrm{g}}$ in the unresolved $\kappa$ model (lower). The 68 and $95 \%$ CL regions are given by the solid and dashed contours, respectively. The best fit and SM points are shown by the black cross and red diamond, respectively. The colour scale indicates the value of the test statistic. 


\begin{tabular}{|c|c|c|c|c|c|}
\hline \multirow{3}{*}{ Parameters } & \multicolumn{4}{|c|}{$\sigma \mathcal{B}(\mathrm{fb})$} & \multirow{3}{*}{$\begin{array}{c}\sigma \mathcal{B} /(\sigma \mathcal{B})_{\mathrm{SM}} \\
\text { Observed (Expected) } \\
\text { Best fit }\end{array}$} \\
\hline & SM prediction & \multicolumn{3}{|c|}{ Observed (Expected) } & \\
\hline & $\left(m_{\mathrm{H}}=125.38 \mathrm{GeV}\right)$ & Best fit & Stat. unc. & Syst. unc. & \\
\hline $\operatorname{ggH} 0 \mathrm{~J}$ low $p_{\mathrm{T}}^{\mathrm{H}}$ & $15.21_{-4.18}^{+4.14}$ & $9.41_{-3.99}^{+3.92}$ & $\begin{array}{l}+3.90 \\
-3.98\end{array}$ & ${ }_{-0.25}^{+0.44}($ & $0.62_{-0.26}^{+0.26}$ \\
\hline $\operatorname{ggH} 0 \mathrm{~J}$ high $p_{\mathrm{T}}^{\mathrm{H}}$ & $44.25_{-4.61}^{+4.84}$ & $58.50_{-7.1}^{+8.1}$ & $\begin{array}{l}+7.70 \\
-6.91\end{array}$ & ${ }_{-1.92}^{+2.50}\left(\begin{array}{c}+1.78 \\
-1.42\end{array}\right)$ & $1.32_{-0.16}^{+0.18}($ \\
\hline $\operatorname{ggH} 1 \mathrm{~J}$ low $p_{\mathrm{T}}^{\mathrm{H}}$ & $16.20_{-2.27}^{+2.25}$ & $13.39_{-5.49}^{+5.58}$ & $\begin{array}{l}+5.52 \\
-5.45\end{array}$ & ${ }_{-0.63}^{+0.80}\left(\begin{array}{c}+0.77 \\
-0.48\end{array}\right)$ & $0.83_{-0.34}^{+0.34}($ \\
\hline $\operatorname{ggH} 1 \mathrm{~J} \operatorname{med} p_{\mathrm{T}}^{\mathrm{H}}$ & $11.23_{-1.55}^{+1.56}$ & $13.66_{-2}^{+2 .}$ & ${ }_{-2.92}^{+2.83}$ & ${ }_{-0.50}^{+0.70}\left(\begin{array}{c}+0.59 \\
-0.45\end{array}\right)$ & $1.22_{-0.26}^{+0.26}($ \\
\hline $\operatorname{ggH} 1 \mathrm{~J}$ high $p_{\mathrm{T}}^{\mathrm{H}}$ & $2.00_{-0.36}^{+0.36}$ & $2.56_{-0.87}^{+0.90}$ & $\begin{array}{l}+0.90 \\
-0.87\end{array}$ & ${ }_{-0.11}^{+0.11}\left(\begin{array}{c}+0.15 \\
-0.19\end{array}\right)$ & $1.28_{-0.44}^{+0.45}($ \\
\hline $\operatorname{ggH} \geq 2 \mathrm{~J}$ low $p_{\mathrm{T}}^{\mathrm{H}}$ & $2.82_{-0.68}^{+0.68}$ & $3.62_{-3.55}^{+3.65}$ & $\begin{array}{l}+3.62 \\
-3.53\end{array}$ & ${ }_{-0.31}^{+0.41}\left(\begin{array}{c}+0.55 \\
-0.55\end{array}\right)$ & $1.29_{-1.26}^{+1.29}\left(\begin{array}{c}+1.32 \\
-1.00\end{array}\right)$ \\
\hline $\operatorname{ggH} \geq 2 \mathrm{~J} \operatorname{med} p_{\mathrm{T}}^{\mathrm{H}}$ & $4.53_{-1.07}^{+1.07}$ & $0.08_{-0.08}^{+2.77}$ & ${ }_{-0.08}^{+2.76}$ & ${ }_{-0.08}^{+0.28}\left({ }_{-0}^{+0}\right.$ & $0.02_{-0.02}^{+0.61}($ \\
\hline $\operatorname{ggH} \geq 2 \mathrm{~J}$ high $p_{\mathrm{T}}^{\mathrm{H}}$ & $2.12_{-0.50}^{+0.49}$ & $0.82_{-0.82}^{+0.92}$ & ${ }_{-0.82}^{+0.88}($ & ${ }_{-0.26}^{+0.26}\left(\begin{array}{c}+0.31 \\
-0.14\end{array}\right)$ & $0.39_{-0.39}^{+0.43}($ \\
\hline ggH VBF-like & $2.22_{-0.52}^{+0.52}$ & $5.86_{-2.59}^{+2.45}$ & ${ }_{-2.55}^{+2.27}($ & ${ }_{-0.48}^{+0.92}\left(\begin{array}{c}+0.71 \\
-0.71\end{array}\right)$ & $2.64_{-1.17}^{+1.10}\left(\begin{array}{c}+1.31 \\
-1.00\end{array}\right)$ \\
\hline ggH BSM & $1.43_{-0.35}^{+0.36}$ & $1.34_{-0.47}^{+0.50}$ & ${ }_{-0.46}^{+0.49}$ & ${ }_{-0.09}^{+0.05}\left(\begin{array}{c}+0.09 \\
-0.05\end{array}\right)$ & $0.94_{-0.33}^{+0.35}\left(\begin{array}{c}+0.41 \\
-0.35\end{array}\right)$ \\
\hline qqH VBF-like & $2.96_{-0.59}^{+0.59}$ & $0.49_{-0.49}^{+1.44}$ & ${ }_{-0.49}^{+1.40}\left(\begin{array}{c}+1.47 \\
-1.47\end{array}\right)$ & ${ }_{-0.34}^{+0.34}\left(\begin{array}{c}+0.25 \\
-0.43\end{array}\right)$ & $0.17_{-0.17}^{+0.49}\left(\begin{array}{c}+0.50 \\
-0.52\end{array}\right)$ \\
\hline qqH VH-like & $1.22_{-0.04}^{+0.05}$ & $1.57_{-1.24}^{+1.20}$ & ${ }_{-1.21}^{+1.19}\left(\begin{array}{c}+1.15 \\
-1.23\end{array}\right)$ & ${ }_{-0.26}^{+0.13}\left(\begin{array}{c}+0.07 \\
-0.04\end{array}\right)$ & $1.29_{-1.01}^{+0.98}\left(\begin{array}{c}+0.94 \\
-1.01\end{array}\right)$ \\
\hline $\mathrm{qqH}$ BSM & $0.37_{-0.02}^{+0.03}$ & $0.52_{-0.22}^{+0.24}($ & +0.24 & ${ }_{-0.01}^{+0.03}\left(\begin{array}{c}+0.03 \\
\end{array}\right)$ & $1.42_{-0.59}^{+0.65}\left({ }^{+0.69}\right)$ \\
\hline WH lep & $0.88_{-0.03}^{+0.03}$ & $1.19_{-0.44}^{+0.49}$ & ${ }_{-0.43}^{+0.48}\left(\begin{array}{c}+0.50 \\
-0.41\end{array}\right)$ & ${ }_{-0.04}^{+0.07}\left(\begin{array}{c}+0.05 \\
-0.05\end{array}\right)$ & $1.35_{-0.49}^{+0.55}\left(\begin{array}{c}+0.57 \\
-0.47\end{array}\right)$ \\
\hline ZH lep & $0.54_{-0.02}^{+0.03}$ & $0.71_{-0.35}^{+0.41}$ & ${ }_{-0.35}^{+0.40}\left(\begin{array}{c}+0.41 \\
-0.35\end{array}\right)$ & ${ }_{-0.03}^{+0.07}\left(\begin{array}{c}+0.06 \\
-0.03\end{array}\right)$ & $1.32_{-0.65}^{+0.76}\left(\begin{array}{c}+0.78 \\
-0.65\end{array}\right)$ \\
\hline $\mathrm{t} \overline{\mathrm{t}} \mathrm{H}$ & $1.13_{-0.11}^{+0.08}$ & $1.13_{-0.39}^{+0.42}\left(_{-}^{+}\right.$ & ${ }_{-0.38}^{+0.42}\left(\begin{array}{c}+0.41 \\
-0.40\end{array}\right)$ & ${ }_{-0.07}^{+0.07}\left(\begin{array}{c}+0.09 \\
-0.05\end{array}\right)$ & $1.00_{-0.35}^{+0.37}\left(\begin{array}{c}+0.37 \\
-0.36\end{array}\right)$ \\
\hline $\mathrm{tH}$ & $0.20_{-0.03}^{+0.01}$ & $1.27_{-0.69}^{+0.76}($ & ${ }_{-0.68}^{+0.75}\left(\begin{array}{c}+0.76 \\
-0.20\end{array}\right)$ & ${ }_{-0.13}^{+0.10}\left(\begin{array}{c}+0.08 \\
-0.08\end{array}\right)$ & $6.24_{-3.37}^{+3.72}\left(\begin{array}{c}+3.73 \\
-1.00\end{array}\right)$ \\
\hline
\end{tabular}

Table 12. Results of the maximal merging scheme STXS fit. The best fit cross sections are shown together with the respective $68 \%$ CL intervals. The uncertainty is decomposed into the systematic and statistical components. The expected uncertainties on the fitted parameters, computed assuming the SM predicted cross section values, are given in brackets. Also listed are the SM predictions for the cross sections and the theoretical uncertainty in those predictions.

principal Higgs boson production mechanisms is performed and found to be compatible with the SM prediction with a $p$-value of $50 \%$. Two different measurements are performed within the simplified template cross section framework, in which 17 and 27 independent kinematic regions are measured simultaneously, with corresponding $p$-values with respect to the SM of 31 and 70\%, respectively. Many of these kinematic regions are measured for the first time, including a simultaneous measurement of Higgs boson production in association with two top quarks in five different regions of the Higgs boson transverse momentum $p_{\mathrm{T}}^{\mathrm{H}}$. Furthermore, several additional measurements are the most precise made in a single channel to date. These include cross sections of vector boson fusion in different kinematic 


\begin{tabular}{|c|c|c|c|c|c|}
\hline \multirow{3}{*}{ Parameters } & \multicolumn{4}{|c|}{$\sigma \mathcal{B}(\mathrm{fb})$} & \multirow{3}{*}{$\begin{array}{c}\sigma \mathcal{B} /(\sigma \mathcal{B})_{\mathrm{SM}} \\
\text { Observed (Expected) } \\
\text { Best fit }\end{array}$} \\
\hline & SM prediction & \multicolumn{3}{|c|}{ Observed (Expected) } & \\
\hline & $\left(m_{\mathrm{H}}=125.38 \mathrm{GeV}\right)$ & Best fit & Stat. unc. & Syst. unc. & \\
\hline $\operatorname{ggH} 0 \mathrm{~J}$ low $p_{\mathrm{T}}^{\mathrm{H}}$ & $15.21_{-4.18}^{+4.14}$ & $9.41_{-4.00}^{+3.91}\left(_{-4.0}^{+4.1}\right.$ & ${ }_{-3.99}^{+3.90}\left(\begin{array}{c}+4.16 \\
-4.05\end{array}\right)$ & ${ }_{-0.30}^{+0.37}\left(\begin{array}{c}+0.50 \\
-0.36\end{array}\right)$ & $0.62_{-0.26}^{+0.26}($ \\
\hline $\operatorname{ggH} 0 \mathrm{~J}$ high $p_{\mathrm{T}}^{\mathrm{H}}$ & $44.25_{-4.61}^{+4.84}$ & $58.46_{-7.17}^{+8.12}\left({ }_{-7}^{+7}\right.$ & ${ }_{-6.91}^{+7.69}\left(\begin{array}{c}+7.66 \\
-7.63\end{array}\right)$ & ${ }_{-1.94}^{+2.60}\left(\begin{array}{c}+1.78 \\
-1.50\end{array}\right)$ & $1.32_{-0.16}^{+0.18}\left(\begin{array}{c}+0.18 \\
-0.18\end{array}\right)$ \\
\hline $\operatorname{ggH} 1 \mathrm{~J}$ low $p_{\mathrm{T}}^{\mathrm{H}}$ & $16.20_{-2.27}^{+2.25}$ & $13.40_{-5.50}^{+5.59}\left(_{-5}^{+5}\right.$ & ${ }_{-5.46}^{+5.53}\left(\begin{array}{c}+5.64 \\
-5.55\end{array}\right)$ & ${ }_{-0.67}^{+0.79}\left(\begin{array}{c}+0.77 \\
-0.56\end{array}\right)$ & $0.83_{-0.34}^{+0.34}\left(\begin{array}{c}+0.35 \\
-0.34\end{array}\right)$ \\
\hline $\operatorname{ggH} 1 \mathrm{~J}$ med $p_{\mathrm{T}}^{\mathrm{H}}$ & $11.23_{-1.55}^{+1.56}$ & $13.80_{-2.94}^{+2.90}\left(_{-3}^{+3}\right.$ & ${ }_{-2.90}^{+2.82}\left(\begin{array}{c}+3.08 \\
-3.37\end{array}\right)$ & ${ }_{-0.51}^{+0.68}\left(\begin{array}{c}+0.59 \\
-0.50\end{array}\right)$ & $1.23_{-0.26}^{+0.26}\left(\begin{array}{c}+0.28 \\
-0.30\end{array}\right)$ \\
\hline $\operatorname{ggH} 1 \mathrm{~J}$ high $p_{\mathrm{T}}^{\mathrm{H}}$ & $2.00_{-0.36}^{+0.36}$ & $2.57_{-0.88}^{+0.94}\left(_{-0 .}^{+0.5}\right.$ & ${ }_{-0.87}^{+0.94}\left(\begin{array}{c}+0.91 \\
-0.88\end{array}\right)$ & ${ }_{-0.12}^{+0.08}\left(\begin{array}{c}+0.13 \\
-0.16\end{array}\right)$ & $1.28_{-0.44}^{+0.47}\left(\begin{array}{c}+0.46 \\
-0.45\end{array}\right)$ \\
\hline $\operatorname{ggH} \geq 2 \mathrm{~J}$ low $p_{\mathrm{T}}^{\mathrm{H}}$ & $2.82_{-0.68}^{+0.68}$ & $3.67_{-3.57}^{+3.63}($ & ${ }_{-3.56}^{+3.62}\left(\begin{array}{c}+3.71 \\
-2.82\end{array}\right)$ & ${ }_{-0.30}^{+0.34}\left(\begin{array}{c}+0.49 \\
-0.49\end{array}\right)$ & $1.30_{-1.27}^{+1.29}\left(\begin{array}{c}+1.33 \\
-1.00\end{array}\right)$ \\
\hline $\operatorname{ggH} \geq 2 \mathrm{~J} \operatorname{med} p_{\mathrm{T}}^{\mathrm{H}}$ & $4.53_{-1.07}^{+1.07}$ & $0.00_{-0.00}^{+2.72}\left(_{-2.8}^{+2 . .}\right.$ & ${ }_{-0.00}^{+2.71}\left(\begin{array}{c}+2.86 \\
-2.78\end{array}\right)$ & ${ }_{-0.00}^{+0.26}\left(\begin{array}{c}+0.45 \\
-0.27\end{array}\right)$ & $0.00_{-0.00}^{+0.60}\left(\begin{array}{c}+0.64 \\
-0.62\end{array}\right)$ \\
\hline $\operatorname{ggH} \geq 2 \mathrm{~J}$ high $p_{\mathrm{T}}^{\mathrm{H}}$ & $2.12_{-0.50}^{+0.49}$ & $0.62_{-0.62}^{+1.06}\left(_{-1.1}^{+1.1}\right.$ & ${ }_{-0.62}^{+1.04}\left(\begin{array}{c}+1.11 \\
-1.10\end{array}\right)$ & ${ }_{-0.17}^{+0.17}\left(\begin{array}{c}+0.30 \\
-0.13\end{array}\right)$ & $0.29_{-0.29}^{+0.50}\left(\begin{array}{c}+0.54 \\
-0.52\end{array}\right)$ \\
\hline $\operatorname{ggH}$ BSM $200<p_{\mathrm{T}}^{\mathrm{H}}<300$ & $1.10_{-0.27}^{+0.28}$ & $1.11_{-0.44}^{+0.47}\left(_{-}^{+}\right.$ & ${ }_{-0.43}^{+0.46}\left(\begin{array}{c}+0.56 \\
-0.45\end{array}\right)$ & ${ }_{-0.07}^{+0.08}\left(\begin{array}{c}+0.05 \\
-0.03\end{array}\right)$ & $1.00_{-0.40}^{+0.42}\left(\begin{array}{c}+0.51 \\
-0.41\end{array}\right)$ \\
\hline $\operatorname{ggH}$ BSM $300<p_{\mathrm{T}}^{\mathrm{H}}<450$ & $0.28_{-0.07}^{+0.07}$ & $0.16_{-0.16}^{+0.19}\left(_{-0.1}^{+0.2}\right.$ & ${ }_{-0.16}^{+0.18}\left(\begin{array}{c}+0.19 \\
-0.18\end{array}\right)$ & ${ }_{-0.02}^{+0.02}\left(\begin{array}{c}+0.03 \\
-0.01\end{array}\right)$ & $0.55_{-0.55}^{+0.66}\left(\begin{array}{c}+0.69 \\
-0.65\end{array}\right)$ \\
\hline $\operatorname{ggH} \mathrm{BSM} p_{\mathrm{T}}^{\mathrm{H}}>450$ & $0.05_{-0.02}^{+0.02}$ & $0.10_{-0.08}^{+0.10}\left({ }_{-0.0}^{+0.1}\right.$ & ${ }_{-0.08}^{+0.09}\left(\begin{array}{c}+0.09 \\
-0.05\end{array}\right)$ & ${ }_{-0.02}^{+0.06}\left(\begin{array}{c}+0.04 \\
-0.04\end{array}\right)$ & $2.16_{-1.69}^{+2.25}\left(\begin{array}{c}+2.19 \\
-1.00\end{array}\right)$ \\
\hline VBF-like low $m_{\mathrm{jj}}$ low $p_{\mathrm{T}}^{\mathrm{Hjj}}$ & $1.59_{-0.48}^{+0.49}$ & $1.31_{-1.13}^{+1.19}\left({ }_{-1.1}^{+1.2}\right.$ & ${ }_{-1.13}^{+1.18}\left(\begin{array}{c}+1.21 \\
-1.16\end{array}\right)$ & ${ }_{-0.09}^{+0.14}\left(\begin{array}{c}+0.13 \\
-0.05\end{array}\right)$ & $0.82_{-0.71}^{+0.75}\left(\begin{array}{c}+0.77 \\
-0.73\end{array}\right)$ \\
\hline VBF-like low $m_{\mathrm{jj}}$ high $p_{\mathrm{T}}^{\mathrm{Hjj}}$ & $1.25_{-0.32}^{+0.35}$ & $3.46_{-1.64}^{+1.76}\left(_{-1.2}^{+1.7}\right.$ & ${ }_{-1.62}^{+1.65}\left(\begin{array}{c}+1.76 \\
-1.25\end{array}\right)$ & ${ }_{-0.25}^{+0.61}\left(\begin{array}{c}+0.32 \\
-0.32\end{array}\right)$ & $2.76_{-1.31}^{+1.40}\left(\begin{array}{c}+1.43 \\
-1.00\end{array}\right)$ \\
\hline VBF-like high $m_{\mathrm{jj}}$ low $p_{\mathrm{T}}^{\mathrm{Hjj}}$ & $1.60_{-0.51}^{+0.45}$ & $0.97_{-0.63}^{+0.64}\left(_{-0.6}^{+0.7}\right.$ & ${ }_{-0.62}^{+0.63}\left(\begin{array}{c}+0.71 \\
-0.63\end{array}\right)$ & ${ }_{-0.07}^{+0.07}\left(\begin{array}{c}+0.11 \\
-0.06\end{array}\right)$ & $0.61_{-0.39}^{+0.40}\left(\begin{array}{c}+0.45 \\
-0.39\end{array}\right)$ \\
\hline VBF-like high $m_{\mathrm{jj}}$ high $p_{\mathrm{T}}^{\mathrm{H} j \mathrm{j}}$ & $0.73_{-0.16}^{+0.16}$ & $0.15_{-0.15}^{+1.08}\left({ }_{-0.7}^{+0.5}\right.$ & ${ }_{-0.15}^{+1.04}\left(\begin{array}{c}+0.92 \\
-0.73\end{array}\right)$ & ${ }_{-0.15}^{+0.28}\left(\begin{array}{c}+0.14 \\
-0.14\end{array}\right)$ & $0.20_{-0.20}^{+1.47}\left(\begin{array}{c}+1.26 \\
-1.00\end{array}\right)$ \\
\hline qqH VH-like & $1.22_{-0.05}^{+0.05}$ & $1.53_{-1.21}^{+1.20}\left(_{-1.2}^{+1.1}\right.$ & ${ }_{-1.20}^{+1.20}\left(\begin{array}{c}+1.12 \\
-1.27\end{array}\right)$ & ${ }_{-0.19}^{+0.11}\left(\begin{array}{c}+0.05 \\
-0.08\end{array}\right)$ & $1.25_{-0.99}^{+0.98}\left(\begin{array}{c}+0.92 \\
-1.04\end{array}\right)$ \\
\hline qqH BSM & $0.37_{-0.02}^{+0.03}$ & $0.51_{-0.22}^{+0.24}\left({ }_{-0.2}^{+0.2}\right.$ & ${ }_{-0.22}^{+0.24}\left(\begin{array}{c}+0.24 \\
-0.24\end{array}\right)$ & ${ }_{-0.01}^{+0.03}\left(\begin{array}{c}+0.03 \\
-0.02\end{array}\right)$ & $1.40_{-0.60}^{+0.66}\left(\begin{array}{c}+0.66 \\
-0.65\end{array}\right)$ \\
\hline WH lep $p_{\mathrm{T}}^{\mathrm{V}}<75$ & $0.47_{-0.02}^{+0.02}$ & $0.71_{-0.54}^{+0.68}\left({ }_{-0.4}^{+0 .}\right.$ & ${ }_{-0.54}^{+0.68}\left(\begin{array}{c}+0.75 \\
-0.47\end{array}\right)$ & ${ }_{-0.05}^{+0.05}\left(\begin{array}{c}+0.05 \\
-0.05\end{array}\right)$ & $1.51_{-1.15}^{+1.45}\left(\begin{array}{c}+1.60 \\
-1.00\end{array}\right)$ \\
\hline WH lep $75<p_{\mathrm{T}}^{\mathrm{V}}<150$ & $0.29_{-0.02}^{+0.02}$ & $0.44_{-0.32}^{+0.37}\left(C_{-0.2}^{+0.3}\right.$ & ${ }_{-0.32}^{+0.37}\left(\begin{array}{c}+0.37 \\
-0.29\end{array}\right)$ & ${ }_{-0.02}^{+0.03}\left(\begin{array}{c}+0.02 \\
-0.02\end{array}\right)$ & $1.49_{-1.08}^{+1.26}\left(\begin{array}{c}+1.25 \\
-1.00\end{array}\right)$ \\
\hline WH lep $p_{\mathrm{T}}^{\mathrm{V}}>150$ & $0.12_{-0.01}^{+0.01}$ & $0.12_{-0.09}^{+0.12}\left(_{-0.1}^{+0.1}\right.$ & ${ }_{-0.09}^{+0.12}\left(\begin{array}{c}+0.13 \\
-0.10\end{array}\right)$ & ${ }_{-0.00}^{+0.01}\left(\begin{array}{c}+0.01 \\
-0.01\end{array}\right)$ & $0.98_{-0.76}^{+0.98}\left(\begin{array}{c}+1.05 \\
-0.79\end{array}\right)$ \\
\hline ZH lep & $0.54_{-0.02}^{+0.03}$ & $0.71_{-0.35}^{+0.41}\left(_{-0 .}^{+0 .}\right.$ & ${ }_{-0.35}^{+0.40}\left(\begin{array}{c}+0.41 \\
-0.35\end{array}\right)$ & ${ }_{-0.03}^{+0.07}\left(\begin{array}{c}+0.04 \\
-0.04\end{array}\right)$ & $1.32_{-0.65}^{+0.76}\left(\begin{array}{c}+0.77 \\
-0.65\end{array}\right)$ \\
\hline $\mathrm{t} \overline{\mathrm{t}} \mathrm{H} p_{\mathrm{T}}^{\mathrm{H}}<60$ & $0.26_{-0.04}^{+0.03}$ & $0.19_{-0.19}^{+0.24}\left(_{-0}^{+0}\right.$ & ${ }_{-0.19}^{+0.24}\left(\begin{array}{c}+0.23 \\
-0.19\end{array}\right)$ & ${ }_{-0.01}^{+0.01}\left(\begin{array}{c}+0.03 \\
-0.03\end{array}\right)$ & $0.73_{-0.73}^{+0.92}\left(\begin{array}{c}+0.89 \\
-0.75\end{array}\right)$ \\
\hline $\mathrm{t} \overline{\mathrm{t}} \mathrm{H} 60<p_{\mathrm{T}}^{\mathrm{H}}<120$ & $0.40_{-0.05}^{+0.04}$ & $0.50_{-0.22}^{+0.26}\left({ }_{-0.2}^{+0.2}\right.$ & ${ }_{-0.22}^{+0.25}\left(\begin{array}{c}+0.28 \\
-0.22\end{array}\right)$ & ${ }_{-0.02}^{+0.04}\left(\begin{array}{c}+0.03 \\
-0.01\end{array}\right)$ & $1.25_{-0.55}^{+0.65}\left(\begin{array}{c}+0.72 \\
-0.55\end{array}\right)$ \\
\hline $\mathrm{t} \overline{\mathrm{t}} \mathrm{H} 120<p_{\mathrm{T}}^{\mathrm{H}}<200$ & $0.29_{-0.04}^{+0.03}$ & $0.24_{-0.14}^{+0.17}\left(C_{-0.1}^{+0.1}\right.$ & ${ }_{-0.14}^{+0.17}\left(\begin{array}{c}+0.17 \\
-0.16\end{array}\right)$ & ${ }_{-0.02}^{+0.02}\left(\begin{array}{c}+0.02 \\
-0.01\end{array}\right)$ & $0.80_{-0.49}^{+0.58}\left(\begin{array}{c}+0.58 \\
-0.53\end{array}\right)$ \\
\hline $\mathrm{t} \overline{\mathrm{t}} \mathrm{H} 200<p_{\mathrm{T}}^{\mathrm{H}}<300$ & $0.12_{-0.02}^{+0.02}$ & $0.11_{-0.09}^{+0.11}\left(_{-0.0}^{+0.1}\right.$ & ${ }_{-0.09}^{+0.11}\left(\begin{array}{c}+0.10 \\
-0.09\end{array}\right)$ & ${ }_{-0.01}^{+0.01}\left(\begin{array}{c}+0.01 \\
-0.01\end{array}\right)$ & $0.92_{-0.73}^{+0.89}\left(\begin{array}{c}+0.81 \\
-0.75\end{array}\right)$ \\
\hline $\mathrm{t} \overline{\mathrm{t}} \mathrm{H} p_{\mathrm{T}}^{\mathrm{H}}>300$ & $0.06_{-0.01}^{+0.01}$ & $0.00_{-0.00}^{+0.08}\left({ }_{-0}^{+0}\right.$ & ${ }_{-0.00}^{+0.08}\left(\begin{array}{c}+0.07 \\
-0.06\end{array}\right)$ & ${ }_{-0.00}^{+0.01}\left(\begin{array}{c}+0.02 \\
-0.02\end{array}\right)$ & $0.00_{-0.00}^{+1.34}\left(\begin{array}{c}+1.21 \\
-1.00\end{array}\right)$ \\
\hline $\mathrm{tH}$ & $0.20_{-0.03}^{+0.01}$ & $1.71_{-0.93}^{+0.71}\left({ }_{-0.2}^{+1.6}\right.$ & ${ }_{-0.92}^{+0.70}\left(\begin{array}{c}+1.00 \\
-0.20\end{array}\right)$ & ${ }_{-0.13}^{+0.13}\left(\begin{array}{c}+0.11 \\
-0.11\end{array}\right)$ & $8.38_{-4.55}^{+3.48}\left(\begin{array}{c}+4.93 \\
-1.00\end{array}\right)$ \\
\hline
\end{tabular}

Table 13. Results of the minimal merging scheme STXS fit. The best fit cross sections are shown together with the respective $68 \% \mathrm{CL}$ intervals. The uncertainty is decomposed into the systematic and statistical components. The expected uncertainties on the fitted parameters, computed assuming the SM predicted cross section values, are given in brackets. Also listed are the SM predictions for the cross sections and the theoretical uncertainty in those predictions. 
regions, gluon fusion in association with jets, and the region of gluon fusion production with $p_{\mathrm{T}}^{\mathrm{H}}>200 \mathrm{GeV}$, which is particularly sensitive to physics beyond the SM. The gluon fusion cross section with $p_{\mathrm{T}}^{\mathrm{H}}>200 \mathrm{GeV}$ is found to be consistent with the SM, with a measured value of $0.9_{-0.3}^{+0.4}$ relative to the SM prediction. An upper limit on the rate of Higgs boson production in association with a single top quark is also presented. The observed (expected) limit at 95\% confidence level is found to be 14 (8) times the SM prediction. All other results, such as measurements of the Higgs boson's couplings to vector bosons and to fermions, are also in agreement with the SM expectations.

\section{Acknowledgments}

We congratulate our colleagues in the CERN accelerator departments for the excellent performance of the LHC and thank the technical and administrative staffs at CERN and at other CMS institutes for their contributions to the success of the CMS effort. In addition, we gratefully acknowledge the computing centres and personnel of the Worldwide LHC Computing Grid and other centres for delivering so effectively the computing infrastructure essential to our analyses. We also gratefully acknowledge the LHC Higgs Working Group for its role in developing stage 1.2 of the simplified template cross section framework. Finally, we acknowledge the enduring support for the construction and operation of the LHC, the CMS detector, and the supporting computing infrastructure provided by the following funding agencies: BMBWF and FWF (Austria); FNRS and FWO (Belgium); CNPq, CAPES, FAPERJ, FAPERGS, and FAPESP (Brazil); MES (Bulgaria); CERN; CAS, MoST, and NSFC (China); COLCIENCIAS (Colombia); MSES and CSF (Croatia); RIF (Cyprus); SENESCYT (Ecuador); MoER, ERC PUT and ERDF (Estonia); Academy of Finland, MEC, and HIP (Finland); CEA and CNRS/IN2P3 (France); BMBF, DFG, and HGF (Germany); GSRT (Greece); NKFIA (Hungary); DAE and DST (India); IPM (Iran); SFI (Ireland); INFN (Italy); MSIP and NRF (Republic of Korea); MES (Latvia); LAS (Lithuania); MOE and UM (Malaysia); BUAP, CINVESTAV, CONACYT, LNS, SEP, and UASLP-FAI (Mexico); MOS (Montenegro); MBIE (New Zealand); PAEC (Pakistan); MSHE and NSC (Poland); FCT (Portugal); JINR (Dubna); MON, RosAtom, RAS, RFBR, and NRC KI (Russia); MESTD (Serbia); SEIDI, CPAN, PCTI, and FEDER (Spain); MOSTR (Sri Lanka); Swiss Funding Agencies (Switzerland); MST (Taipei); ThEPCenter, IPST, STAR, and NSTDA (Thailand); TUBITAK and TAEK (Turkey); NASU (Ukraine); STFC (U.K.); DOE and NSF (U.S.A.).

Individuals have received support from the Marie-Curie programme and the European Research Council and Horizon 2020 Grant, contract Nos. 675440, 724704, 752730, and 765710 (European Union); the Leventis Foundation; the Alfred P. Sloan Foundation; the Alexander von Humboldt Foundation; the Belgian Federal Science Policy Office; the Fonds pour la Formation à la Recherche dans l'Industrie et dans l'Agriculture (FRIA-Belgium); the Agentschap voor Innovatie door Wetenschap en Technologie (IWTBelgium); the F.R.S.-FNRS and FWO (Belgium) under the "Excellence of Science EOS" - be.h project n. 30820817; the Beijing Municipal Science \& Technology Commission, No. Z191100007219010; the Ministry of Education, Youth and Sports (MEYS) of 
the Czech Republic; the Deutsche Forschungsgemeinschaft (DFG), under Germany's Excellence Strategy — EXC 2121 "Quantum Universe" — 390833306, and under project number 400140256 - GRK2497; the Lendület ("Momentum") Programme and the János Bolyai Research Scholarship of the Hungarian Academy of Sciences, the New National Excellence Program ÚNKP, the NKFIA research grants 123842, 123959, 124845, 124850, 125105, 128713, 128786, and 129058 (Hungary); the Council of Science and Industrial Research, India; the Ministry of Science and Higher Education and the National Science Center, contracts Opus 2014/15/B/ST2/03998 and 2015/19/B/ST2/02861 (Poland); the National Priorities Research Program by Qatar National Research Fund; the Ministry of Science and Higher Education, project no. 0723-2020-0041 (Russia); the Programa Estatal de Fomento de la Investigación Científica y Técnica de Excelencia María de Maeztu, grant MDM-20150509 and the Programa Severo Ochoa del Principado de Asturias; the Thalis and Aristeia programmes cofinanced by EU-ESF and the Greek NSRF; the Rachadapisek Sompot Fund for Postdoctoral Fellowship, Chulalongkorn University and the Chulalongkorn Academic into Its 2nd Century Project Advancement Project (Thailand); the Kavli Foundation; the Nvidia Corporation; the SuperMicro Corporation; the Welch Foundation, contract C-1845; and the Weston Havens Foundation (U.S.A.).

Open Access. This article is distributed under the terms of the Creative Commons Attribution License (CC-BY 4.0), which permits any use, distribution and reproduction in any medium, provided the original author(s) and source are credited.

\section{References}

[1] ATLAS collaboration, Observation of a new particle in the search for the Standard Model Higgs boson with the ATLAS detector at the LHC, Phys. Lett. B 716 (2012) 1 [arXiv: 1207.7214] [INSPIRE].

[2] CMS collaboration, Observation of a new boson at a mass of $125 \mathrm{GeV}$ with the CMS experiment at the LHC, Phys. Lett. B 716 (2012) 30 [arXiv:1207.7235] [InSPIRE].

[3] CMS collaboration, Observation of a new boson with mass near $125 \mathrm{GeV}$ in pp collisions at $\sqrt{s}=7$ and $8 \mathrm{TeV}$, JHEP 06 (2013) 081 [arXiv:1303.4571] [INSPIRE].

[4] CMS collaboration, Observation of t $\bar{t} H$ production, Phys. Rev. Lett. 120 (2018) 231801 [arXiv: 1804.02610] [INSPIRE].

[5] ATLAS collaboration, Observation of Higgs boson production in association with a top quark pair at the LHC with the ATLAS detector, Phys. Lett. B $\mathbf{7 8 4}$ (2018) 173 [arXiv: 1806.00425] [INSPIRE].

[6] CMS collaboration, Observation of Higgs boson decay to bottom quarks, Phys. Rev. Lett. 121 (2018) 121801 [arXiv:1808.08242] [INSPIRE].

[7] ATLAS collaboration, Observation of $H \rightarrow b \bar{b}$ decays and $V H$ production with the ATLAS detector, Phys. Lett. B 786 (2018) 59 [arXiv:1808.08238] [INSPIRE].

[8] CMS collaboration, Combined measurements of Higgs boson couplings in proton-proton collisions at $\sqrt{s}=13 \mathrm{TeV}$, Eur. Phys. J. C 79 (2019) 421 [arXiv:1809.10733] [INSPIRE]. 
[9] ATLAS collaboration, Combined measurements of Higgs boson production and decay using up to $80 \mathrm{fb}^{-1}$ of proton-proton collision data at $\sqrt{\mathrm{s}}=13 \mathrm{TeV}$ collected with the ATLAS experiment, Phys. Rev. D 101 (2020) 012002 [arXiv: 1909.02845] [INSPIRE].

[10] LHC Higgs Cross Section Working Group, Handbook of LHC Higgs cross sections: 4. Deciphering the nature of the Higgs sector, in CERN Yellow Reports: Monographs 2, CERN, Geneva Switzerland (2017) [arXiv: 1610.07922] [inSPIRE].

[11] CMS collaboration, Observation of the diphoton decay of the Higgs boson and measurement of its properties, Eur. Phys. J. C $\mathbf{7 4}$ (2014) 3076 [arXiv: 1407.0558] [INSPIRE].

[12] CMS collaboration, Measurements of Higgs boson properties in the diphoton decay channel in proton-proton collisions at $\sqrt{s}=13 \mathrm{TeV}$, JHEP 11 (2018) 185 [arXiv:1804.02716] [INSPIRE].

[13] M. Cepeda et al., Report from Working Group 2: Higgs Physics at the HL-LHC and HE-LHC, in CERN Yellow Reports: Monographs 7, A. Dainese, M. Mangano, A.B. Meyer, A. Nisati, G. Salam and M.A. Vesterinen eds., CERN, Geneva Switzerland (2019), p. 221 [arXiv: 1902.00134] [INSPIRE].

[14] LHC Higgs Cross Section Working Group, Handbook of LHC Higgs cross sections: 3. Higgs Properties. Report of the LHC Higgs Cross Section Working Group, in CERN Yellow Reports: Monographs, CERN, Geneva Switzerland (2013) [arXiv:1307.1347] [INSPIRE].

[15] CMS collaboration, HEPdata record for this analysis, (2021) [https://doi.org/10.17182/hepdata.102459].

[16] CMS collaboration, The CMS trigger system, 2017 JINST 12 P01020 [arXiv:1609.02366] [INSPIRE].

[17] CMS collaboration, The CMS high level trigger, Eur. Phys. J. C 46 (2006) 605 [hep-ex/0512077] [INSPIRE].

[18] CMS collaboration, Particle-flow reconstruction and global event description with the CMS detector, 2017 JINST 12 P10003 [arXiv:1706. 04965] [INSPIRE].

[19] M. Cacciari, G.P. Salam and G. Soyez, The anti- $k_{t}$ jet clustering algorithm, JHEP 04 (2008) 063 [arXiv: 0802.1189] [INSPIRE].

[20] M. Cacciari, G.P. Salam and G. Soyez, FastJet user manual, Eur. Phys. J. C 72 (2012) 1896 [arXiv:1111.6097] [INSPIRE].

[21] CMS collaboration, Jet energy scale and resolution in the CMS experiment in pp collisions at $8 \mathrm{TeV}, 2017$ JINST 12 P02014 [arXiv: 1607. 03663] [INSPIRE].

[22] CMS collaboration, Performance of missing transverse momentum reconstruction in proton-proton collisions at $\sqrt{s}=13 \mathrm{TeV}$ using the CMS detector, 2019 JINST $14 \mathrm{P} 07004$ [arXiv: 1903.06078] [INSPIRE].

[23] CMS collaboration, The CMS experiment at the CERN LHC, 2008 JINST 3 S08004 [INSPIRE].

[24] CMS collaboration, Measurements of $t \bar{t} H$ Production and the CP Structure of the Yukawa Interaction between the Higgs Boson and Top Quark in the Diphoton Decay Channel, Phys. Rev. Lett. 125 (2020) 061801 [arXiv: 2003.10866] [INSPIRE].

[25] ATLAS collaboration, Measurements of Higgs boson properties in the diphoton decay channel with $36 \mathrm{fb}^{-1}$ of pp collision data at $\sqrt{\mathrm{s}}=13 \mathrm{TeV}$ with the ATLAS detector, Phys. Rev. D 98 (2018) 052005 [arXiv: 1802.04146] [INSPIRE]. 
[26] ATLAS collaboration, Higgs boson production cross-section measurements and their EFT interpretation in the $4 \ell$ decay channel at $\sqrt{s}=13 \mathrm{TeV}$ with the ATLAS detector, Eur. Phys. J. C 80 (2020) 957 [Erratum ibid. 81 (2021) 29] [Erratum ibid. 81 (2021) 398] [arXiv: 2004.03447] [INSPIRE].

[27] ATLAS collaboration, Measurements of gluon-gluon fusion and vector-boson fusion Higgs boson production cross-sections in the $H \rightarrow W W^{*} \rightarrow e \nu \mu \nu$ decay channel in pp collisions at $\sqrt{s}=13 \mathrm{TeV}$ with the ATLAS detector, Phys. Lett. B 789 (2019) 508 [arXiv:1808.09054] [INSPIRE].

[28] ATLAS collaboration, Cross-section measurements of the Higgs boson decaying into a pair of $\tau$-leptons in proton-proton collisions at $\sqrt{s}=13 \mathrm{TeV}$ with the ATLAS detector, Phys. Rev. D 99 (2019) 072001 [arXiv: 1811.08856] [INSPIRE].

[29] CMS collaboration, Measurements of properties of the Higgs boson decaying into the four-lepton final state in pp collisions at $\sqrt{s}=13 \mathrm{TeV}$, JHEP 11 (2017) 047 [arXiv: 1706.09936] [INSPIRE].

[30] CMS collaboration, Measurements of properties of the Higgs boson decaying to a $W$ boson pair in pp collisions at $\sqrt{s}=13 \mathrm{TeV}$, Phys. Lett. B 791 (2019) 96 [arXiv:1806.05246] [INSPIRE].

[31] CMS collaboration, Observation of the Higgs boson decay to a pair of $\tau$ leptons with the CMS detector, Phys. Lett. B 779 (2018) 283 [arXiv:1708.00373] [INSPIRE].

[32] N. Berger et al., Simplified Template Cross Sections - Stage 1.1, LHCHXSWG-2019-003 (2019) [DESY-19-070] [arXiv: 1906 .02754] [inSPIRE].

[33] ATLAS collaboration, Measurements of $W H$ and $Z H$ production in the $H \rightarrow b \bar{b}$ decay channel in pp collisions at $13 \mathrm{TeV}$ with the ATLAS detector, Eur. Phys. J. C 81 (2021) 178 [arXiv: 2007.02873] [INSPIRE].

[34] CMS collaboration, Measurements of production cross sections of the Higgs boson in the four-lepton final state in proton-proton collisions at $\sqrt{s}=13 \mathrm{TeV}$, arXiv:2103.04956 [INSPIRE].

[35] T. Chen and C. Guestrin, XGBoost: A Scalable Tree Boosting System, in proceedings of the 22nd ACM SIGKDD International Conference on Knowledge Discovery and Data Mining, San Francisco, CA, U.S.A., 13-17 August 2016, pp. 785-794 [arXiv:1603.02754] [InSPIRE].

[36] A. Hocker et al., TMVA, Toolkit for Multivariate Data Analysis, in proceedings of the XI International Workshop on Advanced Computing and Analysis Techniques in Physics Research (ACAT), Nikhef, Amsterdam, The Netherlands, 23-27 April 2007, PoS ACAT (2009) 040 [physics/0703039] [InSPIRE].

[37] M. Abadi et al., Tensorflow: A system for large-scale machine learning, in proceedings of the 12th USENIX Symposium on Operating Systems Design and Implementation (OSDI'16), Savannah, GA, U.S.A., 2-4 November 2016, USENIX Association (2016), pp. 265-283 [arXiv: 1605.08695].

[38] CMS collaboration, CMS Luminosity Measurements for the 2016 Data Taking Period, CMS-PAS-LUM-17-001 (2017).

[39] CMS collaboration, CMS luminosity measurement for the 2017 data-taking period at $\sqrt{s}=13 \mathrm{TeV}$, CMS-PAS-LUM-17-004 (2018).

[40] CMS collaboration, CMS luminosity measurement for the 2018 data-taking period at $\sqrt{s}=13 \mathrm{TeV}$, CMS-PAS-LUM-18-002 (2019). 
[41] CMS collaboration, Measurement of the inclusive $W$ and $Z$ production cross sections in $p p$ collisions at $\sqrt{s}=7 \mathrm{TeV}$, JHEP 10 (2011) 132 [arXiv:1107.4789] [INSPIRE].

[42] J. Alwall et al., The automated computation of tree-level and next-to-leading order differential cross sections, and their matching to parton shower simulations, JHEP 07 (2014) 079 [arXiv: 1405.0301] [INSPIRE].

[43] K. Hamilton, P. Nason, E. Re and G. Zanderighi, NNLOPS simulation of Higgs boson production, JHEP 10 (2013) 222 [arXiv:1309.0017] [INSPIRE].

[44] T. Sjöstrand et al., An introduction to PYTHIA 8.2, Comput. Phys. Commun. 191 (2015) 159 [arXiv:1410.3012] [INSPIRE].

[45] CMS collaboration, Event generator tunes obtained from underlying event and multiparton scattering measurements, Eur. Phys. J. C 76 (2016) 155 [arXiv:1512.00815] [INSPIRE].

[46] CMS collaboration, Extraction and validation of a new set of CMS PYTHIA8 tunes from underlying-event measurements, Eur. Phys. J. C 80 (2020) 4 [arXiv:1903.12179] [INSPIRE].

[47] NNPDF collaboration, Parton distributions for the LHC Run II, JHEP 04 (2015) 040 [arXiv: 1410.8849] [INSPIRE].

[48] NNPDF collaboration, Parton distributions from high-precision collider data, Eur. Phys. J. C 77 (2017) 663 [arXiv:1706.00428] [INSPIRE].

[49] P. Nason, A New method for combining NLO QCD with shower Monte Carlo algorithms, JHEP 11 (2004) 040 [hep-ph/0409146] [INSPIRE].

[50] S. Frixione, P. Nason and C. Oleari, Matching NLO QCD computations with parton shower simulations: the POWHEG method, JHEP 11 (2007) 070 [arXiv: 0709. 2092] [INSPIRE].

[51] S. Alioli, P. Nason, C. Oleari and E. Re, NLO Higgs boson production via gluon fusion matched with shower in POWHEG, JHEP 04 (2009) 002 [arXiv: 0812.0578] [INSPIRE].

[52] P. Nason and C. Oleari, NLO Higgs boson production via vector-boson fusion matched with shower in POWHEG, JHEP 02 (2010) 037 [arXiv:0911.5299] [INSPIRE].

[53] S. Alioli, P. Nason, C. Oleari and E. Re, A general framework for implementing NLO calculations in shower Monte Carlo programs: the POWHEG BOX, JHEP 06 (2010) 043 [arXiv: 1002.2581] [INSPIRE].

[54] H.B. Hartanto, B. Jager, L. Reina and D. Wackeroth, Higgs boson production in association with top quarks in the POWHEG BOX, Phys. Rev. D 91 (2015) 094003 [arXiv:1501. 04498] [INSPIRE].

[55] T. Gleisberg et al., Event generation with SHERPA 1.1, JHEP 02 (2009) 007 [arXiv: 0811.4622] [INSPIRE].

[56] GEANT4 collaboration, GEANT4 - a simulation toolkit, Nucl. Instrum. Meth. A 506 (2003) 250 [INSPIRE].

[57] CMS collaboration, Electron and photon reconstruction and identification with the CMS experiment at the CERN LHC, 2021 JINST 16 P05014 [arXiv:2012.06888] [INSPIRE].

[58] CMS collaboration, A measurement of the Higgs boson mass in the diphoton decay channel, Phys. Lett. B 805 (2020) 135425 [arXiv:2002.06398] [INSPIRE].

[59] E. Spyromitros-Xioufis, G. Tsoumakas, W. Groves and I. Vlahavas, Multi-target regression via input space expansion: treating targets as inputs, Mach. Learn. 104 (2016) 55 [arXiv:1211.6581]. 
[60] CMS collaboration, Pileup mitigation at CMS in 13 TeV data, 2020 JINST 15 P09018 [arXiv: 2003. 00503] [INSPIRE].

[61] CMS collaboration, Identification of heavy-flavour jets with the CMS detector in pp collisions at $13 \mathrm{TeV}, 2018$ JINST 13 P05011 [arXiv:1712.07158] [INSPIRE].

[62] CMS collaboration, Performance of electron reconstruction and selection with the CMS detector in proton-proton collisions at $\sqrt{s}=8 \mathrm{TeV}, 2015$ JINST $10 \mathrm{P} 06005$ [arXiv: 1502.02701] [INSPIRE].

[63] CMS collaboration, Performance of the CMS muon detector and muon reconstruction with proton-proton collisions at $\sqrt{s}=13 \mathrm{TeV}, 2018$ JINST $13 \mathrm{P} 06015$ [arXiv:1804.04528] [INSPIRE].

[64] ATLAS collaboration, CP Properties of Higgs Boson Interactions with Top Quarks in the $t \bar{t} H$ and $t H$ Processes Using $H \rightarrow \gamma \gamma$ with the ATLAS Detector, Phys. Rev. Lett. 125 (2020) 061802 [arXiv: 2004.04545] [INSPIRE].

[65] CMS collaboration, Search for direct production of supersymmetric partners of the top quark in the all-jets final state in proton-proton collisions at $\sqrt{s}=13 \mathrm{TeV}$, JHEP 10 (2017) 005 [arXiv: 1707.03316] [INSPIRE].

[66] CMS collaboration, Precise determination of the mass of the Higgs boson and tests of compatibility of its couplings with the standard model predictions using proton collisions at 7 and $8 \mathrm{TeV}$, Eur. Phys. J. C $\mathbf{7 5}$ (2015) 212 [arXiv: 1412.8662] [INSPIRE].

[67] G. Cowan, K. Cranmer, E. Gross and O. Vitells, Asymptotic formulae for likelihood-based tests of new physics, Eur. Phys. J. C 71 (2011) 1554 [Erratum ibid. 73 (2013) 2501] [arXiv: 1007.1727] [INSPIRE].

[68] R.A. Fisher, On the interpretation of $\chi^{2}$ from contingency tables, and the calculation of $p, J$. Royal Stat. Soc. 85 (1922) 87.

[69] P.D. Dauncey, M. Kenzie, N. Wardle and G.J. Davies, Handling uncertainties in background shapes: the discrete profiling method, 2015 JINST 10 P04015 [arXiv:1408.6865] [INSPIRE].

[70] CMS collaboration, Measurements of t $\bar{t}$ differential cross sections in proton-proton collisions at $\sqrt{s}=13 \mathrm{TeV}$ using events containing two leptons, JHEP 02 (2019) 149 [arXiv: 1811.06625] [INSPIRE].

[71] CMS collaboration, Measurements of $t \bar{t}$ cross sections in association with $b$ jets and inclusive jets and their ratio using dilepton final states in pp collisions at $\sqrt{s}=13 \mathrm{TeV}$, Phys. Lett. B 776 (2018) 355 [arXiv: 1705.10141] [INSPIRE].

[72] J. Butterworth et al., PDF4LHC recommendations for LHC Run II, J. Phys. G 43 (2016) 023001 [arXiv: 1510.03865] [INSPIRE].

[73] S. Dulat et al., New parton distribution functions from a global analysis of quantum chromodynamics, Phys. Rev. D 93 (2016) 033006 [arXiv:1506.07443] [INSPIRE].

[74] L.A. Harland-Lang, A.D. Martin, P. Motylinski and R.S. Thorne, Parton distributions in the LHC era: MMHT 2014 PDFs, Eur. Phys. J. C 75 (2015) 204 [arXiv:1412.3989] [InSPIRE].

[75] S. Carrazza, S. Forte, Z. Kassabov, J.I. Latorre and J. Rojo, An unbiased Hessian representation for Monte Carlo PDFs, Eur. Phys. J. C $\mathbf{7 5}$ (2015) 369 [arXiv:1505.06736] [INSPIRE].

[76] J. Gao and P. Nadolsky, A meta-analysis of parton distribution functions, JHEP 07 (2014) 035 [arXiv: 1401.0013] [inSPIRE]. 
[77] CMS collaboration, Jet algorithms performance in $13 \mathrm{TeV}$ data, CMS-PAS-JME-16-003 (2017).

[78] CMS collaboration, Performance of the CMS Level-1 trigger in proton-proton collisions at $\sqrt{s}=13 \mathrm{TeV}, 2020$ JINST $15 \mathrm{P} 10017$ [arXiv: 2006.10165] [INSPIRE].

[79] ATLAS, CMS collaborations and the LHC Higgs Combination Group, Procedure for the LHC Higgs boson search combination in Summer 2011, CMS-NOTE-2011-005

[ATL-PHYS-PUB-2011-11] (2011). 


\section{The CMS collaboration}

Yerevan Physics Institute, Yerevan, Armenia

A.M. Sirunyan ${ }^{\dagger}$, A. Tumasyan

Institut für Hochenergiephysik, Wien, Austria

W. Adam, J.W. Andrejkovic, T. Bergauer, S. Chatterjee, M. Dragicevic, A. Es-

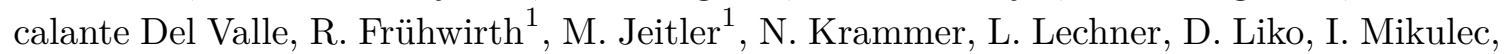
P. Paulitsch, F.M. Pitters, J. Schieck ${ }^{1}$, R. Schöfbeck, M. Spanring, S. Templ, W. Waltenberger, C.-E. Wulz ${ }^{1}$

Institute for Nuclear Problems, Minsk, Belarus

V. Chekhovsky, A. Litomin, V. Makarenko

Universiteit Antwerpen, Antwerpen, Belgium

M.R. Darwish ${ }^{2}$, E.A. De Wolf, X. Janssen, T. Kello ${ }^{3}$, A. Lelek, H. Rejeb Sfar, P. Van Mechelen, S. Van Putte, N. Van Remortel

\section{Vrije Universiteit Brussel, Brussel, Belgium}

F. Blekman, E.S. Bols, J. D'Hondt, J. De Clercq, M. Delcourt, H. El Faham, S. Lowette, S. Moortgat, A. Morton, D. Müller, A.R. Sahasransu, S. Tavernier, W. Van Doninck, P. Van Mulders

Université Libre de Bruxelles, Bruxelles, Belgium

D. Beghin, B. Bilin, B. Clerbaux, G. De Lentdecker, L. Favart, A. Grebenyuk, A.K. Kalsi,

K. Lee, M. Mahdavikhorrami, I. Makarenko, L. Moureaux, L. Pétré, A. Popov, N. Postiau, E. Starling, L. Thomas, M. Vanden Bemden, C. Vander Velde, P. Vanlaer, D. Vannerom, L. Wezenbeek

\section{Ghent University, Ghent, Belgium}

T. Cornelis, D. Dobur, J. Knolle, L. Lambrecht, G. Mestdach, M. Niedziela, C. Roskas, A. Samalan, K. Skovpen, T.T. Tran, M. Tytgat, W. Verbeke, B. Vermassen, M. Vit

Université Catholique de Louvain, Louvain-la-Neuve, Belgium

A. Bethani, G. Bruno, F. Bury, C. Caputo, P. David, C. Delaere, I.S. Donertas, A. Giammanco, K. Jaffel, V. Lemaitre, K. Mondal, J. Prisciandaro, A. Taliercio, M. Teklishyn, P. Vischia, S. Wertz, S. Wuyckens

Centro Brasileiro de Pesquisas Fisicas, Rio de Janeiro, Brazil

G.A. Alves, C. Hensel, A. Moraes

Universidade do Estado do Rio de Janeiro, Rio de Janeiro, Brazil

W.L. Aldá Júnior, M. Alves Gallo Pereira, M. Barroso Ferreira Filho, H. Brandao Malbouisson, W. Carvalho, J. Chinellato ${ }^{4}$, E.M. Da Costa, G.G. Da Silveira ${ }^{5}$, D. De Jesus Damiao, S. Fonseca De Souza, D. Matos Figueiredo, C. Mora Herrera, K. Mota Amarilo, L. Mundim, H. Nogima, P. Rebello Teles, A. Santoro, S.M. Silva Do Amaral, A. Sznajder, M. Thiel, F. Torres Da Silva De Araujo, A. Vilela Pereira 
Universidade Estadual Paulista ${ }^{a}$, Universidade Federal do $\mathrm{ABC}^{b}$, São Paulo, Brazil

C.A. Bernardes ${ }^{a, a}$, L. Calligaris ${ }^{a}$, T.R. Fernandez Perez Tomei $^{a}$, E.M. Gregores ${ }^{a, b}$, D.S. Lemos ${ }^{a}$, P.G. Mercadante ${ }^{a, b}$, S.F. Novaes ${ }^{a}$, Sandra S. Padula ${ }^{a}$

Institute for Nuclear Research and Nuclear Energy, Bulgarian Academy of Sciences, Sofia, Bulgaria

A. Aleksandrov, G. Antchev, R. Hadjiiska, P. Iaydjiev, M. Misheva, M. Rodozov,

M. Shopova, G. Sultanov

University of Sofia, Sofia, Bulgaria

A. Dimitrov, T. Ivanov, L. Litov, B. Pavlov, P. Petkov, A. Petrov

Beihang University, Beijing, China

T. Cheng, W. Fang ${ }^{3}$, Q. Guo, T. Javaid ${ }^{6}$, M. Mittal, H. Wang, L. Yuan

Department of Physics, Tsinghua University, Beijing, China

M. Ahmad, G. Bauer, C. Dozen ${ }^{7}$, Z. Hu, J. Martins ${ }^{8}$, Y. Wang, K. Yi ${ }^{9,10}$

Institute of High Energy Physics, Beijing, China

E. Chapon, G.M. Chen ${ }^{6}$, H.S. Chen ${ }^{6}$, M. Chen, F. Iemmi, A. Kapoor, D. Leggat, H. Liao, Z.-A. Liu ${ }^{6}$, V. Milosevic, F. Monti, M.A. Shahzad ${ }^{6}$, R. Sharma, J. Tao, J. Thomas-wilsker, J. Wang, H. Zhang, J. Zhao

State Key Laboratory of Nuclear Physics and Technology, Peking University, Beijing, China

A. Agapitos, Y. Ban, C. Chen, Q. Huang, A. Levin, Q. Li, M. Lu, X. Lyu, Y. Mao, S.J. Qian, D. Wang, Q. Wang, J. Xiao

Sun Yat-Sen University, Guangzhou, China

Z. You

Institute of Modern Physics and Key Laboratory of Nuclear Physics and Ionbeam Application (MOE) — Fudan University, Shanghai, China

X. $\mathrm{Gao}^{3}, \mathrm{H}$. Okawa

Zhejiang University, Hangzhou, China

M. Xiao

Universidad de Los Andes, Bogota, Colombia

C. Avila, A. Cabrera, C. Florez, J. Fraga, A. Sarkar, M.A. Segura Delgado

Universidad de Antioquia, Medellin, Colombia

J. Mejia Guisao, F. Ramirez, J.D. Ruiz Alvarez, C.A. Salazar González

University of Split, Faculty of Electrical Engineering, Mechanical Engineering and Naval Architecture, Split, Croatia

D. Giljanovic, N. Godinovic, D. Lelas, I. Puljak 
University of Split, Faculty of Science, Split, Croatia

Z. Antunovic, M. Kovac, T. Sculac

Institute Rudjer Boskovic, Zagreb, Croatia

V. Brigljevic, D. Ferencek, D. Majumder, M. Roguljic, A. Starodumov ${ }^{11}$, T. Susa

University of Cyprus, Nicosia, Cyprus

A. Attikis, E. Erodotou, A. Ioannou, G. Kole, M. Kolosova, S. Konstantinou, J. Mousa,

C. Nicolaou, F. Ptochos, P.A. Razis, H. Rykaczewski, H. Saka

Charles University, Prague, Czech Republic

M. Finger ${ }^{12}$, M. Finger Jr. ${ }^{12}$, A. Kveton

Escuela Politecnica Nacional, Quito, Ecuador

E. Ayala

Universidad San Francisco de Quito, Quito, Ecuador

E. Carrera Jarrin

Academy of Scientific Research and Technology of the Arab Republic of Egypt, Egyptian Network of High Energy Physics, Cairo, Egypt

H. Abdalla ${ }^{13}$, A.A. Abdelalim ${ }^{14,15}$

Center for High Energy Physics (CHEP-FU), Fayoum University, El-Fayoum, Egypt

M.A. Mahmoud, Y. Mohammed

National Institute of Chemical Physics and Biophysics, Tallinn, Estonia

S. Bhowmik, A. Carvalho Antunes De Oliveira, R.K. Dewanjee, K. Ehataht, M. Kadastik, J. Pata, M. Raidal, C. Veelken

Department of Physics, University of Helsinki, Helsinki, Finland

P. Eerola, L. Forthomme, H. Kirschenmann, K. Osterberg, M. Voutilainen

Helsinki Institute of Physics, Helsinki, Finland

S. Bharthuar, E. Brücken, F. Garcia, J. Havukainen, M.S. Kim, R. Kinnunen, T. Lampén,

K. Lassila-Perini, S. Lehti, T. Lindén, M. Lotti, L. Martikainen, J. Ott, H. Siikonen,

E. Tuominen, J. Tuominiemi

Lappeenranta University of Technology, Lappeenranta, Finland

P. Luukka, H. Petrow, T. Tuuva

IRFU, CEA, Université Paris-Saclay, Gif-sur-Yvette, France

C. Amendola, M. Besancon, F. Couderc, M. Dejardin, D. Denegri, J.L. Faure, F. Ferri,

S. Ganjour, A. Givernaud, P. Gras, G. Hamel de Monchenault, P. Jarry, B. Lenzi, E. Locci,

J. Malcles, J. Rander, A. Rosowsky, M.Ö. Sahin, A. Savoy-Navarro ${ }^{16}$, M. Titov, G.B. Yu

Laboratoire Leprince-Ringuet, CNRS/IN2P3, Ecole Polytechnique, Institut Polytechnique de Paris, Palaiseau, France

S. Ahuja, F. Beaudette, M. Bonanomi, A. Buchot Perraguin, P. Busson, A. Cappati, C. Charlot, O. Davignon, B. Diab, G. Falmagne, S. Ghosh, R. Granier de Cassagnac, 
A. Hakimi, I. Kucher, M. Nguyen, C. Ochando, P. Paganini, J. Rembser, R. Salerno, J.B. Sauvan, Y. Sirois, A. Zabi, A. Zghiche

\section{Université de Strasbourg, CNRS, IPHC UMR 7178, Strasbourg, France}

J.-L. Agram ${ }^{17}$, J. Andrea, D. Apparu, D. Bloch, G. Bourgatte, J.-M. Brom, E.C. Chabert, C. Collard, D. Darej, J.-C. Fontaine ${ }^{17}$, U. Goerlach, C. Grimault, A.-C. Le Bihan, E. Nibigira, P. Van Hove

Institut de Physique des 2 Infinis de Lyon (IP2I ), Villeurbanne, France

E. Asilar, S. Beauceron, C. Bernet, G. Boudoul, C. Camen, A. Carle, N. Chanon,

D. Contardo, P. Depasse, H. El Mamouni, J. Fay, S. Gascon, M. Gouzevitch, B. Ille, Sa. Jain, I.B. Laktineh, H. Lattaud, A. Lesauvage, M. Lethuillier, L. Mirabito, K. Shchablo, L. Torterotot, G. Touquet, M. Vander Donckt, S. Viret

\section{Georgian Technical University, Tbilisi, Georgia}

A. Khvedelidze ${ }^{12}$, I. Lomidze, Z. Tsamalaidze ${ }^{12}$

\section{RWTH Aachen University, I. Physikalisches Institut, Aachen, Germany}

L. Feld, K. Klein, M. Lipinski, D. Meuser, A. Pauls, M.P. Rauch, N. Röwert, J. Schulz, M. Teroerde

\section{RWTH Aachen University, III. Physikalisches Institut A, Aachen, Germany}

D. Eliseev, M. Erdmann, P. Fackeldey, B. Fischer, S. Ghosh, T. Hebbeker, K. Hoepfner, F. Ivone, H. Keller, L. Mastrolorenzo, M. Merschmeyer, A. Meyer, G. Mocellin, S. Mondal, S. Mukherjee, D. Noll, A. Novak, T. Pook, A. Pozdnyakov, Y. Rath, H. Reithler, J. Roemer, A. Schmidt, S.C. Schuler, A. Sharma, S. Wiedenbeck, S. Zaleski

RWTH Aachen University, III. Physikalisches Institut B, Aachen, Germany

C. Dziwok, G. Flügge, W. Haj Ahmad ${ }^{18}$, O. Hlushchenko, T. Kress, A. Nowack, C. Pistone, O. Pooth, D. Roy, H. Sert, A. Stahl ${ }^{19}$, T. Ziemons

\section{Deutsches Elektronen-Synchrotron, Hamburg, Germany}

H. Aarup Petersen, M. Aldaya Martin, P. Asmuss, I. Babounikau, S. Baxter, O. Behnke, A. Bermúdez Martínez, A.A. Bin Anuar, K. Borras ${ }^{20}$, V. Botta, D. Brunner, A. Campbell, A. Cardini, C. Cheng, S. Consuegra Rodríguez, G. Correia Silva, V. Danilov, L. Didukh, G. Eckerlin, D. Eckstein, L.I. Estevez Banos, O. Filatov, E. Gallo ${ }^{21}$, A. Geiser, A. Giraldi, A. Grohsjean, M. Guthoff, A. Jafari ${ }^{22}$, N.Z. Jomhari, H. Jung, A. Kasem ${ }^{20}$, M. Kasemann, H. Kaveh, C. Kleinwort, D. Krücker, W. Lange, J. Lidrych, K. Lipka, W. Lohmann ${ }^{23}$, R. Mankel, I.-A. Melzer-Pellmann, J. Metwally, A.B. Meyer, M. Meyer, J. Mnich, A. Mussgiller, Y. Otarid, D. Pérez Adán, D. Pitzl, A. Raspereza, B. Ribeiro Lopes, J. Rübenach, A. Saggio, A. Saibel, M. Savitskyi, V. Scheurer, C. Schwanenberger ${ }^{21}$, A. Singh, R.E. Sosa Ricardo, D. Stafford, N. Tonon, O. Turkot, M. Van De Klundert, R. Walsh, D. Walter, Y. Wen, K. Wichmann, C. Wissing, S. Wuchterl

\section{University of Hamburg, Hamburg, Germany}

R. Aggleton, S. Bein, L. Benato, A. Benecke, P. Connor, K. De Leo, M. Eich, F. Feindt, A. Fröhlich, C. Garbers, E. Garutti, P. Gunnellini, J. Haller, A. Hinzmann, G. Kasieczka, 
R. Klanner, R. Kogler, T. Kramer, V. Kutzner, J. Lange, T. Lange, A. Lobanov, A. Malara, A. Nigamova, K.J. Pena Rodriguez, O. Rieger, P. Schleper, M. Schröder, J. Schwandt, D. Schwarz, J. Sonneveld, H. Stadie, G. Steinbrück, A. Tews, B. Vormwald, I. Zoi

Karlsruher Institut fuer Technologie, Karlsruhe, Germany

J. Bechtel, T. Berger, E. Butz, R. Caspart, T. Chwalek, W. De Boer ${ }^{\dagger}$, A. Dierlamm, A. Droll, K. El Morabit, N. Faltermann, M. Giffels, J.o. Gosewisch, A. Gottmann, F. Hartmann ${ }^{19}$, C. Heidecker, U. Husemann, I. Katkov ${ }^{24}$, P. Keicher, R. Koppenhöfer, S. Maier, M. Metzler, S. Mitra, Th. Müller, M. Neukum, A. Nürnberg, G. Quast, K. Rabbertz, J. Rauser, D. Savoiu, M. Schnepf, D. Seith, I. Shvetsov, H.J. Simonis, R. Ulrich, J. Van Der Linden, R.F. Von Cube, M. Wassmer, M. Weber, S. Wieland, R. Wolf, S. Wozniewski, S. Wunsch

Institute of Nuclear and Particle Physics (INPP), NCSR Demokritos, Aghia Paraskevi, Greece

G. Anagnostou, P. Asenov, G. Daskalakis, T. Geralis, A. Kyriakis, D. Loukas, A. Stakia

National and Kapodistrian University of Athens, Athens, Greece

M. Diamantopoulou, D. Karasavvas, G. Karathanasis, P. Kontaxakis, C.K. Koraka, A. Manousakis-katsikakis, A. Panagiotou, I. Papavergou, N. Saoulidou, K. Theofilatos, E. Tziaferi, K. Vellidis, E. Vourliotis

National Technical University of Athens, Athens, Greece

G. Bakas, K. Kousouris, I. Papakrivopoulos, G. Tsipolitis, A. Zacharopoulou

University of Ioánnina, Ioánnina, Greece

I. Evangelou, C. Foudas, P. Gianneios, P. Katsoulis, P. Kokkas, N. Manthos, I. Papadopoulos, J. Strologas

MTA-ELTE Lendület CMS Particle and Nuclear Physics Group, Eötvös Loránd University, Budapest, Hungary

M. Csanad, K. Farkas, M.M.A. Gadallah ${ }^{25}$, S. Lökös ${ }^{26}$, P. Major, K. Mandal, A. Mehta, G. Pasztor, A.J. Rádl, O. Surányi, G.I. Veres

Wigner Research Centre for Physics, Budapest, Hungary

M. Bartók ${ }^{27}$, G. Bencze, C. Hajdu, D. Horvath ${ }^{28}$, F. Sikler, V. Veszpremi, G. Vesztergombi ${ }^{\dagger}$

Institute of Nuclear Research ATOMKI, Debrecen, Hungary

S. Czellar, J. Karancsi ${ }^{27}$, J. Molnar, Z. Szillasi, D. Teyssier

Institute of Physics, University of Debrecen, Debrecen, Hungary

P. Raics, Z.L. Trocsanyi ${ }^{29}$, B. Ujvari

Eszterhazy Karoly University, Karoly Robert Campus, Gyongyos, Hungary

T. Csorgo ${ }^{30}$, F. Nemes ${ }^{30}$, T. Novak

Indian Institute of Science (IISc), Bangalore, India

J.R. Komaragiri, D. Kumar, L. Panwar, P.C. Tiwari 
National Institute of Science Education and Research, HBNI, Bhubaneswar, India

S. Bahinipati ${ }^{31}$, D. Dash, C. Kar, P. Mal, T. Mishra, V.K. Muraleedharan Nair Bindhu ${ }^{32}$, A. Nayak ${ }^{32}$, P. Saha, N. Sur, S.K. Swain, D. Vats ${ }^{32}$

Panjab University, Chandigarh, India

S. Bansal, S.B. Beri, V. Bhatnagar, G. Chaudhary, S. Chauhan, N. Dhingra ${ }^{33}$, R. Gupta, A. Kaur, M. Kaur, S. Kaur, P. Kumari, M. Meena, K. Sandeep, J.B. Singh, A.K. Virdi

University of Delhi, Delhi, India

A. Ahmed, A. Bhardwaj, B.C. Choudhary, R.B. Garg, M. Gola, S. Keshri, A. Kumar, M. Naimuddin, P. Priyanka, K. Ranjan, A. Shah

Saha Institute of Nuclear Physics, HBNI, Kolkata, India

M. Bharti ${ }^{34}$, R. Bhattacharya, S. Bhattacharya, D. Bhowmik, S. Dutta, S. Dutta, B. Gomber ${ }^{35}$, M. Maity ${ }^{36}$, S. Nandan, P. Palit, P.K. Rout, G. Saha, B. Sahu, S. Sarkar, M. Sharan, B. Singh ${ }^{34}$, S. Thakur ${ }^{34}$

Indian Institute of Technology Madras, Madras, India

P.K. Behera, S.C. Behera, P. Kalbhor, A. Muhammad, R. Pradhan, P.R. Pujahari, A. Sharma, A.K. Sikdar

Bhabha Atomic Research Centre, Mumbai, India

D. Dutta, V. Jha, V. Kumar, D.K. Mishra, K. Naskar ${ }^{37}$, P.K. Netrakanti, L.M. Pant, P. Shukla

Tata Institute of Fundamental Research-A, Mumbai, India

T. Aziz, S. Dugad, M. Kumar, U. Sarkar

Tata Institute of Fundamental Research-B, Mumbai, India

S. Banerjee, S. Bhattacharya, R. Chudasama, M. Guchait, S. Karmakar, S. Kumar, G. Majumder, K. Mazumdar, S. Mukherjee

Indian Institute of Science Education and Research (IISER), Pune, India

K. Alpana, S. Dube, B. Kansal, S. Pandey, A. Rane, A. Rastogi, S. Sharma

Department of Physics, Isfahan University of Technology, Isfahan, Iran

H. Bakhshiansohi ${ }^{38}$, M. Zeinali ${ }^{39}$

Institute for Research in Fundamental Sciences (IPM), Tehran, Iran

S. Chenarani ${ }^{40}$, S.M. Etesami, M. Khakzad, M. Mohammadi Najafabadi

University College Dublin, Dublin, Ireland

M. Grunewald

INFN Sezione di Bari ${ }^{a}$, Università di Bari ${ }^{b}$, Politecnico di Bari ${ }^{c}$, Bari, Italy

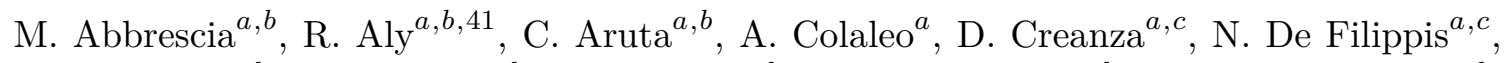
M. De Palma ${ }^{a, b}$, A. Di Florio ${ }^{a, b}$, A. Di Pilato ${ }^{a, b}$, W. Elmetenawee ${ }^{a, b}$, L. Fiore $^{a}$, A. Gelmi $^{a, b}$,

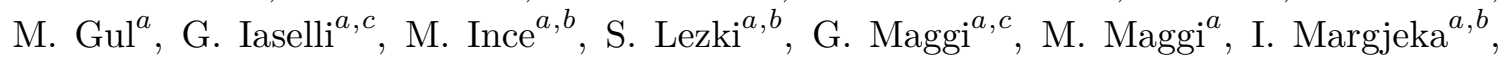


V. Mastrapasqua ${ }^{a, b}$, J.A. Merlin ${ }^{a}$, S. My ${ }^{a, b}$, S. Nuzzo ${ }^{a, b}$, A. Pellecchia $^{a, b}$, A. Pompili ${ }^{a, b}$,

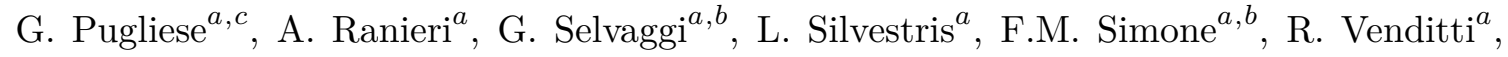
P. Verwilligen ${ }^{a}$

INFN Sezione di Bologna ${ }^{a}$, Università di Bologna ${ }^{b}$, Bologna, Italy

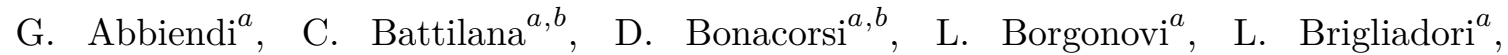
R. Campanini ${ }^{a, b}$, P. Capiluppi ${ }^{a, b}$, A. Castro ${ }^{a, b}$, F.R. Cavallo ${ }^{a}$, M. Cuffiani $^{a, b}$, G.M. Dallavalle ${ }^{a}$, T. Diotalevi ${ }^{a, b}$, F. Fabbri ${ }^{a}$, A. Fanfani ${ }^{a, b}$, P. Giacomelli $^{a}$, L. Giommi $^{a, b}{ }^{,}$ C. Grandi ${ }^{a}$, L. Guiducci ${ }^{a, b}$, S. Lo Meo ${ }^{a, 42}$, L. Lunerti ${ }^{a, b}$, S. Marcellini ${ }^{a}$, G. Masetti ${ }^{a}$, F.L. Navarria ${ }^{a, b}$, A. Perrotta ${ }^{a}$, F. Primavera ${ }^{a, b}$, A.M. Rossi ${ }^{a, b}$, T. Rovelli ${ }^{a, b}$, G.P. Siroli ${ }^{a, b}$

INFN Sezione di Catania ${ }^{a}$, Università di Catania ${ }^{b}$, Catania, Italy

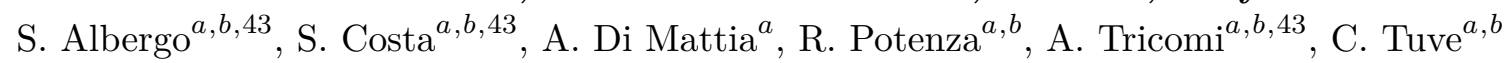

INFN Sezione di Firenze ${ }^{a}$, Università di Firenze ${ }^{b}$, Firenze, Italy

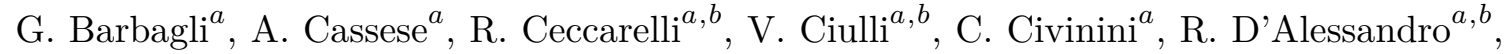

E. Focardi ${ }^{a, b}$, G. Latino ${ }^{a, b}$, P. Lenzi ${ }^{a, b}$, M. Lizzo ${ }^{a, b}$, M. Meschini ${ }^{a}$, S. Paoletti ${ }^{a}$, R. Seidita ${ }^{a, b}$, G. Sguazzoni ${ }^{a}$, L. Viliani $^{a}$

INFN Laboratori Nazionali di Frascati, Frascati, Italy

L. Benussi, S. Bianco, D. Piccolo

INFN Sezione di Genova ${ }^{a}$, Università di Genova ${ }^{b}$, Genova, Italy

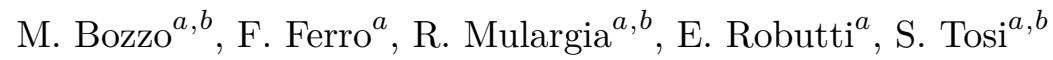

INFN Sezione di Milano-Bicocca $^{a}$, Università di Milano-Bicocca ${ }^{b}$, Milano, Italy

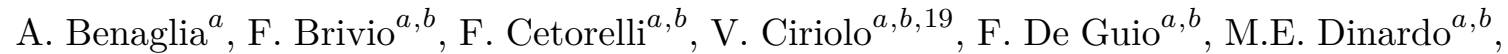
P. Dini ${ }^{a}$, S. Gennai ${ }^{a}$, A. Ghezzi ${ }^{a, b}$, P. Govoni ${ }^{a}, b$, L. Guzzi $^{a, b}$, M. Malberti $^{a}$, S. Malvezzi $^{a}$, A. Massironi ${ }^{a}$, D. Menasce ${ }^{a}$, L. Moroni ${ }^{a}$, M. Paganoni ${ }^{a, b}$, D. Pedrini ${ }^{a}$, S. Ragazzi $^{a, b}$, N. Redaelli ${ }^{a}$, T. Tabarelli de Fatis ${ }^{a, b}$, D. Valsecchi ${ }^{a, b, 19}$, D. Zuolo ${ }^{a, b}$

INFN Sezione di Napoli ${ }^{a}$, Università di Napoli 'Federico II' ${ }^{b}$, Napoli, Italy, Università della Basilicata $^{c}$, Potenza, Italy, Università G. Marconi ${ }^{d}$, Roma, Italy

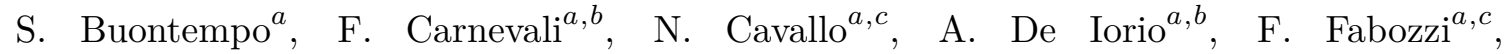

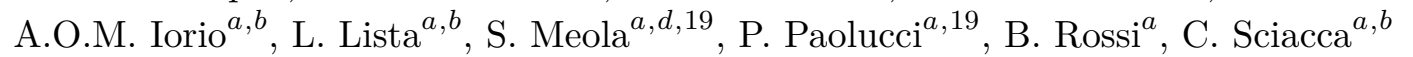

INFN Sezione di Padova ${ }^{a}$, Università di Padova ${ }^{b}$, Padova, Italy, Università di Trento $^{c}$, Trento, Italy

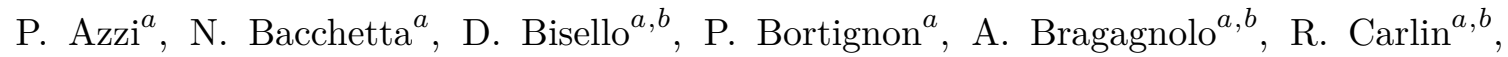

P. Checchia ${ }^{a}$, P. De Castro Manzano ${ }^{a}$, T. Dorigo ${ }^{a}$, U. Dosselli ${ }^{a}$, F. Gasparini ${ }^{a, b}$,

U. Gasparini ${ }^{a, b}$, S.Y. Hoh ${ }^{a, b}$, L. Layer ${ }^{a, 44}$, M. Margoni ${ }^{a, b}$, A.T. Meneguzzo ${ }^{a, b}$, J. Pazzini $^{a, b}$,

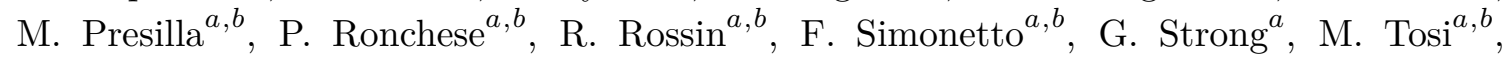

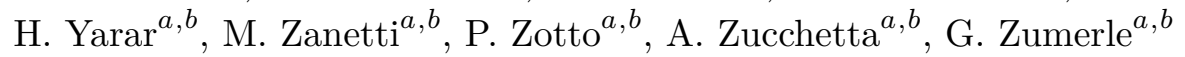

INFN Sezione di Pavia ${ }^{a}$, Università di Pavia ${ }^{b}$, Pavia, Italy

C. Aimè $\grave{a}^{a, b}$ A. Braghieri ${ }^{a}$, S. Calzaferri ${ }^{a, b}$, D. Fiorina ${ }^{a, b}$, P. Montagna ${ }^{a, b}$, S.P. Ratti ${ }^{a, b}$,

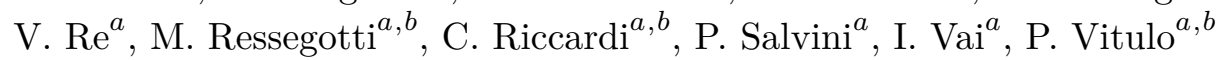


INFN Sezione di Perugia ${ }^{a}$, Università di Perugia ${ }^{b}$, Perugia, Italy G.M. Bilei ${ }^{a}$, D. Ciangottini ${ }^{a, b}$, L. Fanò ${ }^{a, b}$, P. Lariccia ${ }^{a, b}$, M. Magherini $^{b}$, G. Mantovani $^{a, b}{ }^{b}$ V. Mariani ${ }^{a, b}$, M. Menichelli ${ }^{a}$, F. Moscatelli $^{a}$, A. Piccinelli $^{a, b}$, A. Rossi $^{a, b}$, A. Santocchia $^{a, b}$, D. Spiga ${ }^{a}$, T. Tedeschi ${ }^{a, b}$

INFN Sezione di Pisa ${ }^{a}$, Università di Pisa ${ }^{b}$, Scuola Normale Superiore di Pisa ${ }^{c}$, Pisa Italy, Università di Siena ${ }^{d}$, Siena, Italy

P. Azzurri ${ }^{a}$, G. Bagliesi ${ }^{a}$, V. Bertacchi ${ }^{a, c}$, L. Bianchini $^{a}$, T. Boccali ${ }^{a}$, E. Bossini $^{a}{ }^{b}{ }$, R. Castaldi ${ }^{a}$, M.A. Ciocci ${ }^{a, b}$, R. Dell'Orso ${ }^{a}$, M.R. Di Domenico ${ }^{a, d}$, S. Donato $^{a}$, A. Giassi $^{a}$, M.T. Grippo ${ }^{a}$, F. Ligabue ${ }^{a, c}$, E. Manca ${ }^{a, c}$, G. Mandorli ${ }^{a, c}$, A. Messineo ${ }^{a, b}$, F. Palla $^{a}$, S. Parolia ${ }^{a, b}$, G. Ramirez-Sanchez ${ }^{a, c}$, A. Rizzi ${ }^{a, b}$, G. Rolandi ${ }^{a, c}$, S. Roy Chowdhury ${ }^{a, c}$,

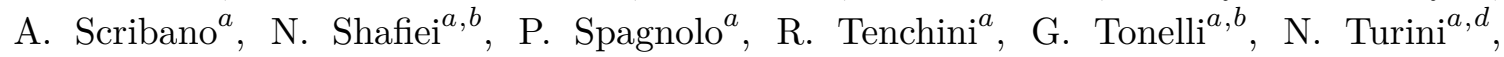
A. Venturi ${ }^{a}$, P.G. Verdini ${ }^{a}$

INFN Sezione di Roma ${ }^{a}$, Sapienza Università di Roma ${ }^{b}$, Rome, Italy

M. Campana ${ }^{a, b}$, F. Cavallari ${ }^{a}$, M. Cipriani ${ }^{a}, b$, D. Del Re ${ }^{a, b}$, E. Di Marco ${ }^{a}$, M. Diemoz $^{a}$, E. Longo $^{a, b}$, P. Meridiani $^{a}$, G. Organtini ${ }^{a, b}$, F. Pandolfi ${ }^{a}$, R. Paramatti $^{a, b}$, C. Quaranta $^{a, b}$,

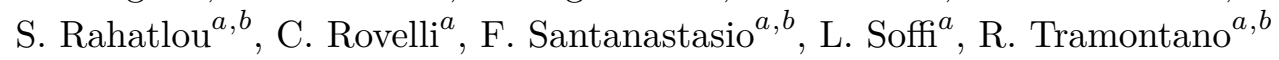

INFN Sezione di Torino ${ }^{a}$, Università di Torino ${ }^{b}$, Torino, Italy, Università del Piemonte Orientale ${ }^{c}$, Novara, Italy

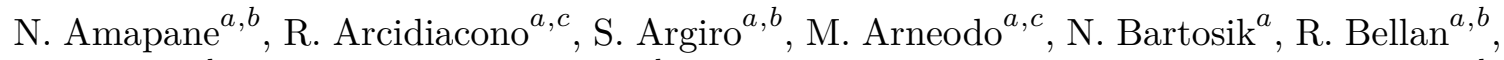

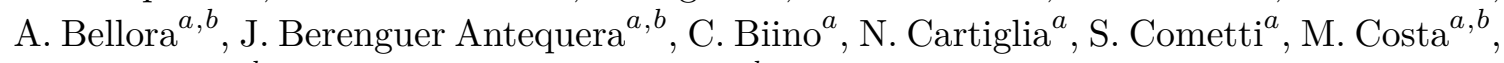
R. Covarelli ${ }^{a, b}$, N. Demaria ${ }^{a}$, B. Kiani ${ }^{a, b}$, F. Legger ${ }^{a}$, C. Mariotti $^{a}$, S. Maselli ${ }^{a}$, E. Migliore $^{a, b}$, E. Monteil ${ }^{a, b}$, M. Monteno ${ }^{a}$, M.M. Obertino ${ }^{a, b}$, G. Ortona $^{a}$, L. Pacher ${ }^{a, b}$, N. Pastrone ${ }^{a}$, M. Pelliccioni ${ }^{a}$, G.L. Pinna Angioni ${ }^{a, b}$, M. Ruspa ${ }^{a, c}$, R. Salvatico $^{a, b}$, K. Shchelina ${ }^{a, b}$, F. Siviero ${ }^{a, b}$, V. Sola ${ }^{a}$, A. Solano ${ }^{a, b}$, D. Soldi ${ }^{a, b}$, A. Staiano $^{a}$, M. Tornago ${ }^{a, b}$, D. Trocino ${ }^{a, b}$, A. Vagnerini

INFN Sezione di Trieste ${ }^{a}$, Università di Trieste ${ }^{b}$, Trieste, Italy

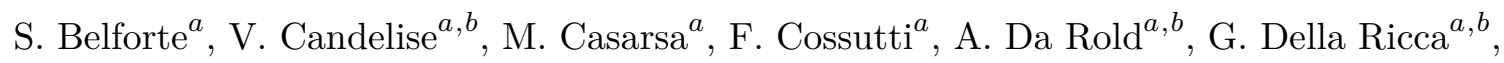
$\mathrm{G}$ Sorrentino ${ }^{a, b}, \mathrm{~F}$. Vazzoler ${ }^{a, b}$

Kyungpook National University, Daegu, Korea

S. Dogra, C. Huh, B. Kim, D.H. Kim, G.N. Kim, J. Kim, J. Lee, S.W. Lee, C.S. Moon, Y.D. Oh, S.I. Pak, B.C. Radburn-Smith, S. Sekmen, Y.C. Yang

Chonnam National University, Institute for Universe and Elementary Particles, Kwangju, Korea

H. Kim, D.H. Moon

Hanyang University, Seoul, Korea

B. Francois, T.J. Kim, J. Park

Korea University, Seoul, Korea

S. Cho, S. Choi, Y. Go, B. Hong, K. Lee, K.S. Lee, J. Lim, J. Park, S.K. Park, J. Yoo 
Kyung Hee University, Department of Physics, Seoul, Republic of Korea

J. Goh, A. Gurtu

Sejong University, Seoul, Korea

H.S. Kim, Y. Kim

Seoul National University, Seoul, Korea

J. Almond, J.H. Bhyun, J. Choi, S. Jeon, J. Kim, J.S. Kim, S. Ko, H. Kwon, H. Lee, S. Lee, B.H. Oh, M. Oh, S.B. Oh, H. Seo, U.K. Yang, I. Yoon

University of Seoul, Seoul, Korea

W. Jang, D. Jeon, D.Y. Kang, Y. Kang, J.H. Kim, S. Kim, B. Ko, J.S.H. Lee, Y. Lee, I.C. Park, Y. Roh, D. Song, I.J. Watson, S. Yang

Yonsei University, Department of Physics, Seoul, Korea

S. Ha, H.D. Yoo

Sungkyunkwan University, Suwon, Korea

Y. Jeong, H. Lee, Y. Lee, I. Yu

College of Engineering and Technology, American University of the Middle East (AUM), Egaila, Kuwait

T. Beyrouthy, Y. Maghrbi

Riga Technical University, Riga, Latvia

V. Veckalns ${ }^{45}$

Vilnius University, Vilnius, Lithuania

M. Ambrozas, A. Juodagalvis, A. Rinkevicius, G. Tamulaitis, A. Vaitkevicius

National Centre for Particle Physics, Universiti Malaya, Kuala Lumpur, Malaysia

N. Bin Norjoharuddeen, W.A.T. Wan Abdullah, M.N. Yusli, Z. Zolkapli

Universidad de Sonora (UNISON), Hermosillo, Mexico

J.F. Benitez, A. Castaneda Hernandez, M. León Coello, J.A. Murillo Quijada, A. Sehrawat, L. Valencia Palomo

Centro de Investigacion y de Estudios Avanzados del IPN, Mexico City, Mexico G. Ayala, H. Castilla-Valdez, I. Heredia-De La Cruz ${ }^{46}$, R. Lopez-Fernandez, C.A. Mondragon Herrera, D.A. Perez Navarro, A. Sanchez-Hernandez

Universidad Iberoamericana, Mexico City, Mexico

S. Carrillo Moreno, C. Oropeza Barrera, M. Ramirez-Garcia, F. Vazquez Valencia

Benemerita Universidad Autonoma de Puebla, Puebla, Mexico

I. Pedraza, H.A. Salazar Ibarguen, C. Uribe Estrada

University of Montenegro, Podgorica, Montenegro

J. Mijuskovic $^{47}$, N. Raicevic 
University of Auckland, Auckland, New Zealand

D. Krofcheck

University of Canterbury, Christchurch, New Zealand

S. Bheesette, P.H. Butler

National Centre for Physics, Quaid-I-Azam University, Islamabad, Pakistan

A. Ahmad, M.I. Asghar, A. Awais, M.I.M. Awan, H.R. Hoorani, W.A. Khan, M.A. Shah, M. Shoaib, M. Waqas

AGH University of Science and Technology Faculty of Computer Science, Electronics and Telecommunications, Krakow, Poland

V. Avati, L. Grzanka, M. Malawski

National Centre for Nuclear Research, Swierk, Poland

H. Bialkowska, M. Bluj, B. Boimska, M. Górski, M. Kazana, M. Szleper, P. Zalewski

Institute of Experimental Physics, Faculty of Physics, University of Warsaw, Warsaw, Poland

K. Bunkowski, K. Doroba, A. Kalinowski, M. Konecki, J. Krolikowski, M. Walczak

Laboratório de Instrumentação e Física Experimental de Partículas, Lisboa, Portugal

M. Araujo, P. Bargassa, D. Bastos, A. Boletti, P. Faccioli, M. Gallinaro, J. Hollar, N. Leonardo, T. Niknejad, M. Pisano, J. Seixas, O. Toldaiev, J. Varela

Joint Institute for Nuclear Research, Dubna, Russia

S. Afanasiev, D. Budkouski, I. Golutvin, I. Gorbunov, V. Karjavine, V. Korenkov, A. Lanev, A. Malakhov, V. Matveev ${ }^{48,49}$, V. Palichik, V. Perelygin, M. Savina, D. Seitova, V. Shalaev, S. Shmatov, S. Shulha, V. Smirnov, O. Teryaev, N. Voytishin, B.S. Yuldashev ${ }^{50}$, A. Zarubin, I. Zhizhin

Petersburg Nuclear Physics Institute, Gatchina (St. Petersburg), Russia

G. Gavrilov, V. Golovtcov, Y. Ivanov, V. Kim ${ }^{51}$, E. Kuznetsova ${ }^{52}$, V. Murzin, V. Oreshkin, I. Smirnov, D. Sosnov, V. Sulimov, L. Uvarov, S. Volkov, A. Vorobyev

Institute for Nuclear Research, Moscow, Russia

Yu. Andreev, A. Dermenev, S. Gninenko, N. Golubev, A. Karneyeu, D. Kirpichnikov, M. Kirsanov, N. Krasnikov, A. Pashenkov, G. Pivovarov, D. Tlisov ${ }^{\dagger}$, A. Toropin

Institute for Theoretical and Experimental Physics named by A.I. Alikhanov of NRC 'Kurchatov Institute', Moscow, Russia

V. Epshteyn, V. Gavrilov, N. Lychkovskaya, A. Nikitenko ${ }^{53}$, V. Popov, A. Spiridonov, A. Stepennov, M. Toms, E. Vlasov, A. Zhokin

Moscow Institute of Physics and Technology, Moscow, Russia

T. Aushev 
National Research Nuclear University 'Moscow Engineering Physics Institute' (MEPhI), Moscow, Russia

O. Bychkova, R. Chistov ${ }^{54}$, M. Danilov ${ }^{55}$, P. Parygin, S. Polikarpov ${ }^{54}$

P.N. Lebedev Physical Institute, Moscow, Russia

V. Andreev, M. Azarkin, I. Dremin, M. Kirakosyan, A. Terkulov

Skobeltsyn Institute of Nuclear Physics, Lomonosov Moscow State University, Moscow, Russia

A. Belyaev, E. Boos, V. Bunichev, M. Dubinin ${ }^{56}$, L. Dudko, A. Ershov, A. Gribushin, V. Klyukhin, O. Kodolova, I. Lokhtin, S. Obraztsov, S. Petrushanko, V. Savrin

Novosibirsk State University (NSU), Novosibirsk, Russia

V. Blinov ${ }^{57}$, T. Dimova ${ }^{57}$, L. Kardapoltsev ${ }^{57}$, A. Kozyrev ${ }^{57}$, I. Ovtin ${ }^{57}$, Y. Skovpen ${ }^{57}$

Institute for High Energy Physics of National Research Centre 'Kurchatov Institute', Protvino, Russia

I. Azhgirey, I. Bayshev, D. Elumakhov, V. Kachanov, D. Konstantinov, P. Mandrik, V. Petrov, R. Ryutin, S. Slabospitskii, A. Sobol, S. Troshin, N. Tyurin, A. Uzunian, A. Volkov

National Research Tomsk Polytechnic University, Tomsk, Russia

A. Babaev, V. Okhotnikov

Tomsk State University, Tomsk, Russia

V. Borchsh, V. Ivanchenko, E. Tcherniaev

University of Belgrade: Faculty of Physics and VINCA Institute of Nuclear Sciences, Belgrade, Serbia

P. Adzic ${ }^{58}$, M. Dordevic, P. Milenovic, J. Milosevic

Centro de Investigaciones Energéticas Medioambientales y Tecnológicas (CIEMAT), Madrid, Spain

M. Aguilar-Benitez, J. Alcaraz Maestre, A. Álvarez Fernández, I. Bachiller, M. Barrio Luna, Cristina F. Bedoya, C.A. Carrillo Montoya, M. Cepeda, M. Cerrada, N. Colino, B. De La Cruz, A. Delgado Peris, J.P. Fernández Ramos, J. Flix, M.C. Fouz, O. Gonzalez Lopez, S. Goy Lopez, J.M. Hernandez, M.I. Josa, J. León Holgado, D. Moran, Á. Navarro Tobar, A. Pérez-Calero Yzquierdo, J. Puerta Pelayo, I. Redondo, L. Romero, S. Sánchez Navas, L. Urda Gómez, C. Willmott

Universidad Autónoma de Madrid, Madrid, Spain

J.F. de Trocóniz, R. Reyes-Almanza

Universidad de Oviedo, Instituto Universitario de Ciencias y Tecnologías Espaciales de Asturias (ICTEA), Oviedo, Spain

B. Alvarez Gonzalez, J. Cuevas, C. Erice, J. Fernandez Menendez, S. Folgueras, I. Gonzalez Caballero, E. Palencia Cortezon, C. Ramón Álvarez, J. Ripoll Sau, V. Rodríguez Bouza, A. Trapote, N. Trevisani 
Instituto de Física de Cantabria (IFCA), CSIC-Universidad de Cantabria, Santander, Spain

J.A. Brochero Cifuentes, I.J. Cabrillo, A. Calderon, B. Chazin Quero, J. Duarte Campderros, M. Fernandez, C. Fernandez Madrazo, P.J. Fernández Manteca, A. García Alonso, G. Gomez, C. Martinez Rivero, P. Martinez Ruiz del Arbol, F. Matorras, P. Matorras Cuevas, J. Piedra Gomez, C. Prieels, T. Rodrigo, A. Ruiz-Jimeno, L. Scodellaro, I. Vila, J.M. Vizan Garcia

\section{University of Colombo, Colombo, Sri Lanka}

M.K. Jayananda, B. Kailasapathy ${ }^{59}$, D.U.J. Sonnadara, D.D.C Wickramarathna

\section{University of Ruhuna, Department of Physics, Matara, Sri Lanka}

W.G.D. Dharmaratna, K. Liyanage, N. Perera, N. Wickramage

\section{CERN, European Organization for Nuclear Research, Geneva, Switzerland}

T.K. Aarrestad, D. Abbaneo, J. Alimena, E. Auffray, G. Auzinger, J. Baechler, P. Baillon ${ }^{\dagger}$, D. Barney, J. Bendavid, M. Bianco, A. Bocci, T. Camporesi, M. Capeans Garrido, G. Cerminara, S.S. Chhibra, L. Cristella, D. d'Enterria, A. Dabrowski, N. Daci, A. David, A. De Roeck, M.M. Defranchis, M. Deile, M. Dobson, M. Dünser, N. Dupont, A. ElliottPeisert, N. Emriskova, F. Fallavollita ${ }^{60}$, D. Fasanella, S. Fiorendi, A. Florent, G. Franzoni, W. Funk, S. Giani, D. Gigi, K. Gill, F. Glege, L. Gouskos, M. Haranko, J. Hegeman, Y. Iiyama, V. Innocente, T. James, P. Janot, J. Kaspar, J. Kieseler, M. Komm, N. Kratochwil, C. Lange, S. Laurila, P. Lecoq, K. Long, C. Lourenço, L. Malgeri, S. Mallios, M. Mannelli, A.C. Marini, F. Meijers, S. Mersi, E. Meschi, F. Moortgat, M. Mulders, S. Orfanelli, L. Orsini, F. Pantaleo, L. Pape, E. Perez, M. Peruzzi, A. Petrilli, G. Petrucciani, A. Pfeiffer, M. Pierini, D. Piparo, M. Pitt, H. Qu, T. Quast, D. Rabady, A. Racz, M. Rieger, M. Rovere, H. Sakulin, J. Salfeld-Nebgen, S. Scarfi, C. Schäfer, C. Schwick, M. Selvaggi, A. Sharma, P. Silva, W. Snoeys, P. Sphicas ${ }^{61}$, S. Summers, V.R. Tavolaro, D. Treille, A. Tsirou, G.P. Van Onsem, M. Verzetti, J. Wanczyk ${ }^{62}$, K.A. Wozniak, W.D. Zeuner

\section{Paul Scherrer Institut, Villigen, Switzerland}

L. Caminada ${ }^{63}$, A. Ebrahimi, W. Erdmann, R. Horisberger, Q. Ingram, H.C. Kaestli, D. Kotlinski, U. Langenegger, M. Missiroli, T. Rohe

ETH Zurich - Institute for Particle Physics and Astrophysics (IPA), Zurich, Switzerland

K. Androsov ${ }^{62}$, M. Backhaus, P. Berger, A. Calandri, N. Chernyavskaya, A. De Cosa, G. Dissertori, M. Dittmar, M. Donegà, C. Dorfer, F. Eble, T.A. Gómez Espinosa, C. Grab, D. Hits, W. Lustermann, A.-M. Lyon, R.A. Manzoni, C. Martin Perez, M.T. Meinhard, F. Micheli, F. Nessi-Tedaldi, J. Niedziela, F. Pauss, V. Perovic, G. Perrin, S. Pigazzini, M.G. Ratti, M. Reichmann, C. Reissel, T. Reitenspiess, B. Ristic, D. Ruini, D.A. Sanz Becerra, M. Schönenberger, V. Stampf, J. Steggemann ${ }^{62}$, R. Wallny, D.H. Zhu

\section{Universität Zürich, Zurich, Switzerland}

C. Amsler ${ }^{64}$, P. Bärtschi, C. Botta, D. Brzhechko, M.F. Canelli, K. Cormier, A. De Wit, R. Del Burgo, J.K. Heikkilä, M. Huwiler, A. Jofrehei, B. Kilminster, S. Leontsinis, 
A. Macchiolo, P. Meiring, V.M. Mikuni, U. Molinatti, I. Neutelings, G. Rauco, A. Reimers,

P. Robmann, S. Sanchez Cruz, K. Schweiger, Y. Takahashi

National Central University, Chung-Li, Taiwan

C. Adloff ${ }^{65}$, C.M. Kuo, W. Lin, A. Roy, T. Sarkar ${ }^{36}$, S.S. Yu

National Taiwan University (NTU), Taipei, Taiwan

L. Ceard, Y. Chao, K.F. Chen, P.H. Chen, W.-S. Hou, Y.y. Li, R.-S. Lu, E. Paganis,

A. Psallidas, A. Steen, E. Yazgan, P.r. Yu

Chulalongkorn University, Faculty of Science, Department of Physics, Bangkok, Thailand

B. Asavapibhop, C. Asawatangtrakuldee, N. Srimanobhas

Çukurova University, Physics Department, Science and Art Faculty, Adana, Turkey

F. Boran, S. Damarseckin ${ }^{66}$, Z.S. Demiroglu, F. Dolek, I. Dumanoglu ${ }^{67}$, E. Eskut, Y. Guler,

E. Gurpinar Guler ${ }^{68}$, I. Hos $^{69}$, C. Isik, O. Kara, A. Kayis Topaksu, U. Kiminsu, G. Onengut,

K. Ozdemir ${ }^{70}$, A. Polatoz, A.E. Simsek, B. Tali ${ }^{71}$, U.G. Tok, S. Turkcapar, I.S. Zorbakir,

C. Zorbilmez

Middle East Technical University, Physics Department, Ankara, Turkey

B. Isildak ${ }^{72}$, G. Karapinar ${ }^{73}$, K. Ocalan ${ }^{74}$, M. Yalvac ${ }^{75}$

Bogazici University, Istanbul, Turkey

B. Akgun, I.O. Atakisi, E. Gülmez, M. Kaya ${ }^{76}$, O. Kaya ${ }^{77}$, Ö. Özçelik, S. Tekten ${ }^{78}$, E.A. Yetkin ${ }^{79}$

Istanbul Technical University, Istanbul, Turkey

A. Cakir, K. Cankocak ${ }^{67}$, Y. Komurcu, S. Sen ${ }^{80}$

Istanbul University, Istanbul, Turkey

S. Cerci ${ }^{71}$, B. Kaynak, S. Ozkorucuklu, D. Sunar Cerci ${ }^{71}$

Institute for Scintillation Materials of National Academy of Science of Ukraine, Kharkov, Ukraine

B. Grynyov

National Scientific Center, Kharkov Institute of Physics and Technology, Kharkov, Ukraine

L. Levchuk

University of Bristol, Bristol, U.K.

D. Anthony, E. Bhal, S. Bologna, J.J. Brooke, A. Bundock, E. Clement, D. Cussans,

H. Flacher, J. Goldstein, G.P. Heath, H.F. Heath, L. Kreczko, B. Krikler, S. Paramesvaran,

S. Seif El Nasr-Storey, V.J. Smith, N. Stylianou ${ }^{81}$, R. White 
Rutherford Appleton Laboratory, Didcot, U.K.

K.W. Bell, A. Belyaev ${ }^{82}$, C. Brew, R.M. Brown, D.J.A. Cockerill, K.V. Ellis, K. Harder, S. Harper, J. Linacre, K. Manolopoulos, D.M. Newbold, E. Olaiya, D. Petyt, T. Reis, T. Schuh, C.H. Shepherd-Themistocleous, I.R. Tomalin, T. Williams

Imperial College, London, U.K.

R. Bainbridge, P. Bloch, S. Bonomally, J. Borg, S. Breeze, O. Buchmuller, V. Cepaitis, G.S. Chahal ${ }^{83}$, D. Colling, P. Dauncey, G. Davies, J. Davies, M. Della Negra, S. Fayer, G. Fedi, G. Hall, M.H. Hassanshahi, G. Iles, J. Langford, L. Lyons, A.-M. Magnan, S. Malik, A. Martelli, J. Nash ${ }^{84}$, M. Pesaresi, D.M. Raymond, A. Richards, A. Rose, E. Scott, C. Seez, A. Shtipliyski, A. Tapper, K. Uchida, T. Virdee ${ }^{19}$, N. Wardle, S.N. Webb, D. Winterbottom, A.G. Zecchinelli, S.C. Zenz

Brunel University, Uxbridge, U.K.

K. Coldham, J.E. Cole, A. Khan, P. Kyberd, I.D. Reid, L. Teodorescu, S. Zahid

Baylor University, Waco, U.S.A.

S. Abdullin, A. Brinkerhoff, B. Caraway, J. Dittmann, K. Hatakeyama, A.R. Kanuganti, B. McMaster, N. Pastika, S. Sawant, C. Smith, C. Sutantawibul, J. Wilson

Catholic University of America, Washington, DC, U.S.A.

R. Bartek, A. Dominguez, R. Uniyal, A.M. Vargas Hernandez

The University of Alabama, Tuscaloosa, U.S.A.

A. Buccilli, S.I. Cooper, D. Di Croce, S.V. Gleyzer, C. Henderson, C.U. Perez, P. Rumerio ${ }^{85}$, C. West

\section{Boston University, Boston, U.S.A.}

A. Akpinar, A. Albert, D. Arcaro, C. Cosby, Z. Demiragli, E. Fontanesi, D. Gastler, J. Rohlf, K. Salyer, D. Sperka, D. Spitzbart, I. Suarez, A. Tsatsos, S. Yuan, D. Zou

Brown University, Providence, U.S.A.

G. Benelli, B. Burkle, X. Coubez ${ }^{20}$, D. Cutts, Y.t. Duh, M. Hadley, U. Heintz, J.M. $\operatorname{Hogan}^{86}$, G. Landsberg, K.T. Lau, J. Lee, M. Lukasik, J. Luo, M. Narain, S. Sagir ${ }^{87}$, E. Usai, W.Y. Wong, X. Yan, D. Yu, W. Zhang

University of California, Davis, Davis, U.S.A.

J. Bonilla, C. Brainerd, R. Breedon, M. Calderon De La Barca Sanchez, M. Chertok, J. Conway, P.T. Cox, R. Erbacher, G. Haza, F. Jensen, O. Kukral, R. Lander, M. Mulhearn, D. Pellett, B. Regnery, D. Taylor, Y. Yao, F. Zhang

University of California, Los Angeles, U.S.A.

M. Bachtis, R. Cousins, A. Datta, D. Hamilton, J. Hauser, M. Ignatenko, M.A. Iqbal, T. Lam, N. Mccoll, W.A. Nash, S. Regnard, D. Saltzberg, B. Stone, V. Valuev

University of California, Riverside, Riverside, U.S.A.

K. Burt, Y. Chen, R. Clare, J.W. Gary, M. Gordon, G. Hanson, G. Karapostoli, O.R. Long, N. Manganelli, M. Olmedo Negrete, W. Si, S. Wimpenny, Y. Zhang 
University of California, San Diego, La Jolla, U.S.A.

J.G. Branson, P. Chang, S. Cittolin, S. Cooperstein, N. Deelen, J. Duarte, R. Gerosa, L. Giannini, D. Gilbert, J. Guiang, R. Kansal, V. Krutelyov, R. Lee, J. Letts, M. Masciovecchio, S. May, M. Pieri, B.V. Sathia Narayanan, V. Sharma, M. Tadel, A. Vartak, F. Würthwein, Y. Xiang, A. Yagil

University of California, Santa Barbara - Department of Physics, Santa Barbara, U.S.A.

N. Amin, C. Campagnari, M. Citron, A. Dorsett, V. Dutta, J. Incandela, M. Kilpatrick, J. Kim, B. Marsh, H. Mei, M. Oshiro, M. Quinnan, J. Richman, U. Sarica, D. Stuart, S. Wang

California Institute of Technology, Pasadena, U.S.A.

A. Bornheim, O. Cerri, I. Dutta, J.M. Lawhorn, N. Lu, J. Mao, H.B. Newman, J. Ngadiuba, T.Q. Nguyen, M. Spiropulu, J.R. Vlimant, C. Wang, S. Xie, Z. Zhang, R.Y. Zhu

Carnegie Mellon University, Pittsburgh, U.S.A.

J. Alison, S. An, M.B. Andrews, P. Bryant, T. Ferguson, A. Harilal, T. Mudholkar, M. Paulini, A. Sanchez

University of Colorado Boulder, Boulder, U.S.A.

J.P. Cumalat, W.T. Ford, E. MacDonald, R. Patel, A. Perloff, K. Stenson, K.A. Ulmer, S.R. Wagner

Cornell University, Ithaca, U.S.A.

J. Alexander, Y. Cheng, J. Chu, D.J. Cranshaw, K. Mcdermott, J. Monroy, J.R. Patterson, D. Quach, J. Reichert, A. Ryd, W. Sun, S.M. Tan, Z. Tao, J. Thom, P. Wittich, M. Zientek

Fermi National Accelerator Laboratory, Batavia, U.S.A.

M. Albrow, M. Alyari, G. Apollinari, A. Apresyan, A. Apyan, S. Banerjee, L.A.T. Bauerdick, D. Berry, J. Berryhill, P.C. Bhat, K. Burkett, J.N. Butler, A. Canepa, G.B. Cerati, H.W.K. Cheung, F. Chlebana, M. Cremonesi, K.F. Di Petrillo, V.D. Elvira, Y. Feng, J. Freeman, Z. Gecse, L. Gray, D. Green, S. Grünendahl, O. Gutsche, R.M. Harris, R. Heller, T.C. Herwig, J. Hirschauer, B. Jayatilaka, S. Jindariani, M. Johnson, U. Joshi, T. Klijnsma, B. Klima, K.H.M. Kwok, S. Lammel, D. Lincoln, R. Lipton, T. Liu, C. Madrid, K. Maeshima, C. Mantilla, D. Mason, P. McBride, P. Merkel, S. Mrenna, S. Nahn, V. O'Dell, V. Papadimitriou, K. Pedro, C. Pena ${ }^{56}$, O. Prokofyev, F. Ravera, A. Reinsvold Hall, L. Ristori, B. Schneider, E. Sexton-Kennedy, N. Smith, A. Soha, W.J. Spalding, L. Spiegel, S. Stoynev, J. Strait, L. Taylor, S. Tkaczyk, N.V. Tran, L. Uplegger, E.W. Vaandering, H.A. Weber

\section{University of Florida, Gainesville, U.S.A.}

D. Acosta, P. Avery, D. Bourilkov, L. Cadamuro, V. Cherepanov, F. Errico, R.D. Field, D. Guerrero, B.M. Joshi, M. Kim, E. Koenig, J. Konigsberg, A. Korytov, K.H. Lo, K. Matchev, N. Menendez, G. Mitselmakher, A. Muthirakalayil Madhu, N. Rawal, D. Rosenzweig, S. Rosenzweig, K. Shi, J. Sturdy, J. Wang, E. Yigitbasi, X. Zuo 
Florida State University, Tallahassee, U.S.A.

T. Adams, A. Askew, D. Diaz, R. Habibullah, V. Hagopian, K.F. Johnson, R. Khurana, T. Kolberg, G. Martinez, H. Prosper, C. Schiber, R. Yohay, J. Zhang

Florida Institute of Technology, Melbourne, U.S.A.

M.M. Baarmand, S. Butalla, T. Elkafrawy ${ }^{88}$, M. Hohlmann, R. Kumar Verma, D. Noonan, M. Rahmani, M. Saunders, F. Yumiceva

University of Illinois at Chicago (UIC), Chicago, U.S.A.

M.R. Adams, H. Becerril Gonzalez, R. Cavanaugh, X. Chen, S. Dittmer, O. Evdokimov, C.E. Gerber, D.A. Hangal, D.J. Hofman, C. Mills, G. Oh, T. Roy, M.B. Tonjes, N. Varelas, J. Viinikainen, X. Wang, Z. Wu, Z. Ye

The University of Iowa, Iowa City, U.S.A.

M. Alhusseini, K. Dilsiz ${ }^{89}$, R.P. Gandrajula, O.K. Köseyan, J.-P. Merlo, A. Mestvirishvili ${ }^{90}$, J. Nachtman, H. Ogul ${ }^{91}$, Y. Onel, A. Penzo, C. Snyder, E. Tiras ${ }^{92}$

Johns Hopkins University, Baltimore, U.S.A.

O. Amram, B. Blumenfeld, L. Corcodilos, J. Davis, M. Eminizer, A.V. Gritsan, S. Kyriacou, P. Maksimovic, J. Roskes, M. Swartz, T.Á. Vámi

The University of Kansas, Lawrence, U.S.A.

J. Anguiano, C. Baldenegro Barrera, P. Baringer, A. Bean, A. Bylinkin, T. Isidori, S. Khalil, J. King, G. Krintiras, A. Kropivnitskaya, C. Lindsey, N. Minafra, M. Murray, C. Rogan, C. Royon, S. Sanders, E. Schmitz, J.D. Tapia Takaki, Q. Wang, J. Williams, G. Wilson

Kansas State University, Manhattan, U.S.A.

S. Duric, A. Ivanov, K. Kaadze, D. Kim, Y. Maravin, T. Mitchell, A. Modak, K. Nam

Lawrence Livermore National Laboratory, Livermore, U.S.A.

F. Rebassoo, D. Wright

University of Maryland, College Park, U.S.A.

E. Adams, A. Baden, O. Baron, A. Belloni, S.C. Eno, N.J. Hadley, S. Jabeen, R.G. Kellogg,

T. Koeth, A.C. Mignerey, S. Nabili, M. Seidel, A. Skuja, L. Wang, K. Wong

Massachusetts Institute of Technology, Cambridge, U.S.A.

D. Abercrombie, G. Andreassi, R. Bi, S. Brandt, W. Busza, I.A. Cali, Y. Chen, M. D'Alfonso, J. Eysermans, G. Gomez Ceballos, M. Goncharov, P. Harris, M. Hu, M. Klute, D. Kovalskyi, J. Krupa, Y.-J. Lee, B. Maier, C. Mironov, C. Paus, D. Rankin, C. Roland, G. Roland, Z. Shi, G.S.F. Stephans, K. Tatar, J. Wang, Z. Wang, B. Wyslouch

University of Minnesota, Minneapolis, U.S.A.

R.M. Chatterjee, A. Evans, P. Hansen, J. Hiltbrand, Sh. Jain, M. Krohn, Y. Kubota, J. Mans, M. Revering, R. Rusack, R. Saradhy, N. Schroeder, N. Strobbe, M.A. Wadud 
University of Nebraska-Lincoln, Lincoln, U.S.A.

K. Bloom, M. Bryson, S. Chauhan, D.R. Claes, C. Fangmeier, L. Finco, F. Golf, J.R. González Fernández, C. Joo, I. Kravchenko, M. Musich, I. Reed, J.E. Siado, G.R. Snow ${ }^{\dagger}$, W. Tabb, F. Yan

State University of New York at Buffalo, Buffalo, U.S.A.

G. Agarwal, H. Bandyopadhyay, L. Hay, I. Iashvili, A. Kharchilava, C. McLean, D. Nguyen, J. Pekkanen, S. Rappoccio, A. Williams

Northeastern University, Boston, U.S.A.

G. Alverson, E. Barberis, C. Freer, Y. Haddad, A. Hortiangtham, J. Li, G. Madigan, B. Marzocchi, D.M. Morse, V. Nguyen, T. Orimoto, A. Parker, L. Skinnari, A. TishelmanCharny, T. Wamorkar, B. Wang, A. Wisecarver, D. Wood

Northwestern University, Evanston, U.S.A.

S. Bhattacharya, J. Bueghly, Z. Chen, A. Gilbert, T. Gunter, K.A. Hahn, N. Odell, M.H. Schmitt, M. Velasco

University of Notre Dame, Notre Dame, U.S.A.

R. Band, R. Bucci, N. Dev, R. Goldouzian, M. Hildreth, K. Hurtado Anampa, C. Jessop,

K. Lannon, N. Loukas, N. Marinelli, I. Mcalister, T. McCauley, F. Meng, K. Mohrman, Y. Musienko ${ }^{48}$, R. Ruchti, P. Siddireddy, M. Wayne, A. Wightman, M. Wolf, M. Zarucki, L. Zygala

The Ohio State University, Columbus, U.S.A.

B. Bylsma, B. Cardwell, L.S. Durkin, B. Francis, C. Hill, M. Nunez Ornelas, K. Wei, B.L. Winer, B.R. Yates

Princeton University, Princeton, U.S.A.

F.M. Addesa, B. Bonham, P. Das, G. Dezoort, P. Elmer, A. Frankenthal, B. Greenberg, N. Haubrich, S. Higginbotham, A. Kalogeropoulos, G. Kopp, S. Kwan, D. Lange, M.T. Lucchini, D. Marlow, K. Mei, I. Ojalvo, J. Olsen, C. Palmer, D. Stickland, C. Tully

University of Puerto Rico, Mayaguez, U.S.A.

S. Malik, S. Norberg

Purdue University, West Lafayette, U.S.A.

A.S. Bakshi, V.E. Barnes, R. Chawla, S. Das, L. Gutay, M. Jones, A.W. Jung, S. Karmarkar, M. Liu, G. Negro, N. Neumeister, G. Paspalaki, C.C. Peng, S. Piperov, A. Purohit, J.F. Schulte, M. Stojanovic ${ }^{16}$, J. Thieman, F. Wang, R. Xiao, W. Xie

Purdue University Northwest, Hammond, U.S.A.

J. Dolen, N. Parashar

Rice University, Houston, U.S.A.

A. Baty, M. Decaro, S. Dildick, K.M. Ecklund, S. Freed, P. Gardner, F.J.M. Geurts, A. Kumar, W. Li, B.P. Padley, R. Redjimi, W. Shi, A.G. Stahl Leiton, S. Yang, L. Zhang, Y. Zhang 
University of Rochester, Rochester, U.S.A.

A. Bodek, P. de Barbaro, R. Demina, J.L. Dulemba, C. Fallon, T. Ferbel, M. Galanti, A. Garcia-Bellido, O. Hindrichs, A. Khukhunaishvili, E. Ranken, R. Taus

Rutgers, The State University of New Jersey, Piscataway, U.S.A.

B. Chiarito, J.P. Chou, A. Gandrakota, Y. Gershtein, E. Halkiadakis, A. Hart, M. Heindl, E. Hughes, S. Kaplan, O. Karacheban ${ }^{23}$, I. Laflotte, A. Lath, R. Montalvo, K. Nash, M. Osherson, S. Salur, S. Schnetzer, S. Somalwar, R. Stone, S.A. Thayil, S. Thomas, H. Wang

University of Tennessee, Knoxville, U.S.A.

H. Acharya, A.G. Delannoy, S. Spanier

Texas A\&M University, College Station, U.S.A.

O. Bouhali ${ }^{93}$, M. Dalchenko, A. Delgado, R. Eusebi, J. Gilmore, T. Huang, T. Kamon ${ }^{94}$, H. Kim, S. Luo, S. Malhotra, R. Mueller, D. Overton, D. Rathjens, A. Safonov

Texas Tech University, Lubbock, U.S.A.

N. Akchurin, J. Damgov, V. Hegde, S. Kunori, K. Lamichhane, S.W. Lee, T. Mengke, S. Muthumuni, T. Peltola, I. Volobouev, Z. Wang, A. Whitbeck

Vanderbilt University, Nashville, U.S.A.

E. Appelt, S. Greene, A. Gurrola, W. Johns, A. Melo, H. Ni, K. Padeken, F. Romeo, P. Sheldon, S. Tuo, J. Velkovska

University of Virginia, Charlottesville, U.S.A.

M.W. Arenton, B. Cox, G. Cummings, J. Hakala, R. Hirosky, M. Joyce, A. Ledovskoy, A. Li, C. Neu, B. Tannenwald, E. Wolfe

Wayne State University, Detroit, U.S.A.

N. Poudyal, P. Thapa

University of Wisconsin - Madison, Madison, WI, U.S.A.

K. Black, T. Bose, J. Buchanan, C. Caillol, S. Dasu, I. De Bruyn, P. Everaerts, F. Fienga, C. Galloni, H. He, M. Herndon, A. Hervé, U. Hussain, A. Lanaro, A. Loeliger, R. Loveless, J. Madhusudanan Sreekala, A. Mallampalli, A. Mohammadi, D. Pinna, A. Savin, V. Shang, V. Sharma, W.H. Smith, D. Teague, S. Trembath-reichert, W. Vetens

$\dagger$ : Deceased

1: Also at Vienna University of Technology, Vienna, Austria

2: Also at Institute of Basic and Applied Sciences, Faculty of Engineering, Arab Academy for Science, Technology and Maritime Transport, Alexandria, Egypt, Alexandria, Egypt

3: Also at Université Libre de Bruxelles, Bruxelles, Belgium

4: Also at Universidade Estadual de Campinas, Campinas, Brazil

5: Also at Federal University of Rio Grande do Sul, Porto Alegre, Brazil

6: Also at University of Chinese Academy of Sciences, Beijing, China

7: Also at Department of Physics, Tsinghua University, Beijing, China, Beijing, China

8: Also at UFMS, Nova Andradina, Brazil 
9: Also at Nanjing Normal University Department of Physics, Nanjing, China

10: Now at The University of Iowa, Iowa City, U.S.A.

11: Also at Institute for Theoretical and Experimental Physics named by A.I. Alikhanov of NRC 'Kurchatov Institute', Moscow, Russia

12: Also at Joint Institute for Nuclear Research, Dubna, Russia

13: Also at Cairo University, Cairo, Egypt

14: Also at Helwan University, Cairo, Egypt

15: Now at Zewail City of Science and Technology, Zewail, Egypt

16: Also at Purdue University, West Lafayette, U.S.A.

17: Also at Université de Haute Alsace, Mulhouse, France

18: Also at Erzincan Binali Yildirim University, Erzincan, Turkey

19: Also at CERN, European Organization for Nuclear Research, Geneva, Switzerland

20: Also at RWTH Aachen University, III. Physikalisches Institut A, Aachen, Germany

21: Also at University of Hamburg, Hamburg, Germany

22: Also at Department of Physics, Isfahan University of Technology, Isfahan, Iran, Isfahan, Iran

23: Also at Brandenburg University of Technology, Cottbus, Germany

24: Also at Skobeltsyn Institute of Nuclear Physics, Lomonosov Moscow State University, Moscow, Russia

25: Also at Physics Department, Faculty of Science, Assiut University, Assiut, Egypt

26: Also at Eszterhazy Karoly University, Karoly Robert Campus, Gyongyos, Hungary

27: Also at Institute of Physics, University of Debrecen, Debrecen, Hungary, Debrecen, Hungary

28: Also at Institute of Nuclear Research ATOMKI, Debrecen, Hungary

29: Also at MTA-ELTE Lendület CMS Particle and Nuclear Physics Group, Eötvös Loránd University, Budapest, Hungary, Budapest, Hungary

30: Also at Wigner Research Centre for Physics, Budapest, Hungary

31: Also at IIT Bhubaneswar, Bhubaneswar, India, Bhubaneswar, India

32: Also at Institute of Physics, Bhubaneswar, India

33: Also at G.H.G. Khalsa College, Punjab, India

34: Also at Shoolini University, Solan, India

35: Also at University of Hyderabad, Hyderabad, India

36: Also at University of Visva-Bharati, Santiniketan, India

37: Also at Indian Institute of Technology (IIT), Mumbai, India

38: Also at Deutsches Elektronen-Synchrotron, Hamburg, Germany

39: Also at Sharif University of Technology, Tehran, Iran

40: Also at Department of Physics, University of Science and Technology of Mazandaran, Behshahr, Iran

41: Now at INFN Sezione di Bari ${ }^{a}$, Università di Bari ${ }^{b}$, Politecnico di Bari ${ }^{c}$, Bari, Italy

42: Also at Italian National Agency for New Technologies, Energy and Sustainable Economic Development, Bologna, Italy

43: Also at Centro Siciliano di Fisica Nucleare e di Struttura Della Materia, Catania, Italy

44: Also at Università di Napoli 'Federico II', NAPOLI, Italy

45: Also at Riga Technical University, Riga, Latvia, Riga, Latvia

46: Also at Consejo Nacional de Ciencia y Tecnología, Mexico City, Mexico

47: Also at IRFU, CEA, Université Paris-Saclay, Gif-sur-Yvette, France

48: Also at Institute for Nuclear Research, Moscow, Russia

49: Now at National Research Nuclear University 'Moscow Engineering Physics Institute' (MEPhI), Moscow, Russia

50: Also at Institute of Nuclear Physics of the Uzbekistan Academy of Sciences, Tashkent, Uzbekistan 
51: Also at St. Petersburg State Polytechnical University, St. Petersburg, Russia

52: Also at University of Florida, Gainesville, U.S.A.

53: Also at Imperial College, London, U.K.

54: Also at P.N. Lebedev Physical Institute, Moscow, Russia

55: Also at Moscow Institute of Physics and Technology, Moscow, Russia, Moscow, Russia

56: Also at California Institute of Technology, Pasadena, U.S.A.

57: Also at Budker Institute of Nuclear Physics, Novosibirsk, Russia

58: Also at Faculty of Physics, University of Belgrade, Belgrade, Serbia

59: Also at Trincomalee Campus, Eastern University, Sri Lanka, Nilaveli, Sri Lanka

60: Also at INFN Sezione di Pavia ${ }^{a}$, Università di Pavia ${ }^{b}$, Pavia, Italy, Pavia, Italy

61: Also at National and Kapodistrian University of Athens, Athens, Greece

62: Also at Ecole Polytechnique Fédérale Lausanne, Lausanne, Switzerland

63: Also at Universität Zürich, Zurich, Switzerland

64: Also at Stefan Meyer Institute for Subatomic Physics, Vienna, Austria, Vienna, Austria

65: Also at Laboratoire d'Annecy-le-Vieux de Physique des Particules, IN2P3-CNRS, Annecyle-Vieux, France

66: Also at Şirnak University, Sirnak, Turkey

67: Also at Near East University, Research Center of Experimental Health Science, Nicosia, Turkey

68: Also at Konya Technical University, Konya, Turkey

69: Also at Istanbul University - Cerraphasa, Faculty of Engineering, Istanbul, Turkey

70: Also at Piri Reis University, Istanbul, Turkey

71: Also at Adiyaman University, Adiyaman, Turkey

72: Also at Ozyegin University, Istanbul, Turkey

73: Also at Izmir Institute of Technology, Izmir, Turkey

74: Also at Necmettin Erbakan University, Konya, Turkey

75: Also at Bozok Universitetesi Rektörlügü, Yozgat, Turkey, Yozgat, Turkey

76: Also at Marmara University, Istanbul, Turkey

77: Also at Milli Savunma University, Istanbul, Turkey

78: Also at Kafkas University, Kars, Turkey

79: Also at Istanbul Bilgi University, Istanbul, Turkey

80: Also at Hacettepe University, Ankara, Turkey

81: Also at Vrije Universiteit Brussel, Brussel, Belgium

82: Also at School of Physics and Astronomy, University of Southampton, Southampton, U.K.

83: Also at IPPP Durham University, Durham, U.K.

84: Also at Monash University, Faculty of Science, Clayton, Australia

85: Also at Università di Torino, TORINO, Italy

86: Also at Bethel University, St. Paul, Minneapolis, U.S.A., St. Paul, U.S.A.

87: Also at Karamanoğlu Mehmetbey University, Karaman, Turkey

88: Also at Ain Shams University, Cairo, Egypt

89: Also at Bingol University, Bingol, Turkey

90: Also at Georgian Technical University, Tbilisi, Georgia

91: Also at Sinop University, Sinop, Turkey

92: Also at Erciyes University, KAYSERI, Turkey

93: Also at Texas A\&M University at Qatar, Doha, Qatar

94: Also at Kyungpook National University, Daegu, Korea, Daegu, Korea 